\title{
A CONVERGENCE THEOREM FOR A CLASS OF NYSTRÖM METHODS FOR WEAKLY SINGULAR INTEGRAL EQUATIONS ON SURFACES IN $\mathbb{R}^{3}$
}

\author{
OSCAR GONZALEZ AND JUN LI
}

\begin{abstract}
A convergence theorem is proved for a class of Nyström methods for weakly singular integral equations on surfaces in three dimensions. Fredholm equations of the second kind as arise in connection with linear elliptic boundary value problems for scalar and vector fields are considered. In contrast to methods based on product integration, coordinate transformation and singularity subtraction, the family of Nyström methods studied here is based on a local polynomial correction determined by an auxiliary system of moment equations. The polynomial correction is shown to remove the weak singularity in the integral equation and provide control over the approximation error. Convergence results for the family of methods are established under minimal regularity assumptions consistent with classic potential theory. Rates of convergence are shown to depend on the regularity of the problem, the degree of the polynomial correction, and the order of the quadrature rule employed in the discretization. As a corollary, a simple method based on singularity subtraction which has been employed by many authors is shown to be convergent.
\end{abstract}

\section{INTRODUCTION}

The method of integral equations has a long and rich history in both the analysis and numerical treatment of boundary value problems $[7,9,14,19,23,24,26,29,31,45$. On the analysis side, the method provides a classic approach to the study of existence and uniqueness questions. On the numerical side, the method provides an alternative formulation which can be efficiently discretized. In the basic approach, a boundary value problem described by a partial differential equation on a domain of interest is reduced to an integral equation on the bounding surface. The unknown scalar or vector field throughout the domain is represented in terms of one or more integral potentials which depend on an unknown surface density. The representation can take various different direct and indirect forms and the integral potentials usually involve kernels that are at least weakly singular. The reduction in dimension makes the method of integral equations extremely attractive, especially for problems on exterior domains. Indeed, the method has been used in many different modern applications in acoustics and electromagnetics [15, 39], hydrodynamics [42, 43], elastostatics [27, 37], and molecular modeling [1,5, 8, 10, 21].

Once a problem has been reduced to an integral equation, various different types of methods are available for its numerical resolution [7, 19, 24, 31, 45. In Galerkin methods, the unknown density and integral equation are projected onto a finitedimensional subspace of functions on the surface, where the projection is defined

Received by the editor December 29, 2011 and, in revised form, June 3, 2013.

2010 Mathematics Subject Classification. Primary 65R20, 65N38; Secondary 45B05, 31B20.

This work was supported by the National Science Foundation. 
through an inner-product on the space. Basis functions for the space may be piecewise polynomials, splines or wavelets with local supports [2, 7, 19, 34, or related eigenfunctions with global supports [6, 17, 18, 22, A method is completed through the specification of appropriate quadrature formulae for the evaluation of double surface integrals in the inner-product. In collocation methods, the unknown density and integral equation are projected similar to before, but the projection is defined through pointwise interpolation on the surface. As a consequence, only single surface integrals arise, which is computationally attractive. In Nyström methods, the integrals in the integral equation are directly approximated by a quadrature rule, which leads to a direct approximation of the unknown density at the quadrature nodes, which can then be interpolated over the surface. For problems with continuous kernels, the Nyström approach leads to simple, efficient and well-understood schemes, and for problems with weakly singular kernels, various modifications are required to handle the singularities, such as product integration, coordinate transformation and singularity subtraction [4, 7, 12, 13, 19, 31, 36, 44, 48,

In this article, we study a class of modified Nyström methods based on local polynomial corrections. The methods studied here share some similarities with product integration and singularity subtraction methods, but have various important differences. The basic approach is to employ standard quadrature weights away from the diagonal of the weakly singular kernel, and generalized or corrected weights near the diagonal. In product integration methods, the corrected weights are defined so as to include the action of the kernel on a mesh-dependent basis of interpolating polynomials. In the methods considered here, the corrected weights include the action of the kernel on a mesh-independent basis of local floating polynomials, and furthermore the weights themselves are expressed as the pointwise values of such a polynomial. The local polynomial that generates the corrected weights is determined through an auxiliary system of moment equations. Such a local polynomial correction is shown to implicitly regularize the weak singularity in the kernel, as would occur in a singularity subtraction method, and provide control over the approximation error.

We establish a convergence theorem for a family of modified Nyström methods for integral equations on surfaces in three dimensions. We restrict attention to Fredholm equations of the second kind with weakly singular kernels as arise in connection with linear elliptic boundary value problems for scalar and vector fields. In contrast to methods based on product integration, coordinate transformation and singularity subtraction, we consider methods based on a local polynomial correction as described above. Convergence results in the standard maximum norm are established under minimal regularity assumptions consistent with classic potential theory. Rates of convergence are shown to depend on the regularity of the surface and data, the degree of the local polynomial correction, and the order of the underlying quadrature rule. In the minimal regularity case, there is no lower bound on the rate, and in the smooth case, there is no upper bound on the rate. Indeed, arbitrarily high rates of convergence can be achieved with local polynomial corrections of arbitrarily high degree. Various important assumptions on the weakly singular kernel and the quadrature rule are discussed in detail. As a corollary of our result, we show that a simple method based on singularity subtraction which has been employed by many authors is convergent under the same assumptions. 
The mathematical theory of convergence of Nyström methods of integral equations of the second kind on regular surfaces is a well-studied subject. The convergence theory for standard methods on problems with continuous kernels is classic 17, 19, 31. Similarly, the theory for product integration methods on problems with weakly singular kernels is also well-established [7, 19, 31, although some of the hypotheses may be difficult to verify in three-dimensional problems. Convergence results for methods based on floating polar coordinate transformations are described in $11,12,48$. Convergence results for methods based on the classic idea of singularity subtraction as introduced by Kantorovich and Krylov [28] have been established for one-dimensional problems in [4, and have been studied for higher-dimensional problems in 44]. Methods similar to those introduced here were considered in [13], but no mathematical convergence results were given. Numerical experiments which illustrate several aspects of the lowest-order method of the family introduced here can be found in 36]. Related convergence results under different regularity assumptions are given in [35].

The presentation is structured as follows. In Section 2 we define the class of boundary integral equations to be studied. We outline various important assumptions on the surface and kernels associated with the equation, and introduce notation and results that will be needed throughout our developments. In Section 3 we define our family of Nyström methods with local polynomial corrections as described above. We outline essential assumptions on the quadrature rule and other elements of the method and then state our main result. In Section 4 we provide a proof of our result. The proof is based on the theory of collectively compact operators and several technical lemmas; the latter are established herein and may be of independent interest.

\section{BOUNDARY INTEGRAL EQUATION}

2.1. Preliminaries, notation. Throughout our developments, we consider a surface $\Gamma$ in $\mathbb{R}^{3}$ consisting of a finite number of disjoint, closed, bounded and orientable components. We invoke the standard assumptions of classic potential theory [23,29] and assume that $\Gamma$ is a Lyapunov surface. Thus:

(L1) there exists a well-defined outward unit normal $\nu(x)$ and tangent plane $T_{x} \Gamma$ at every $x \in \Gamma$,

(L2) there exists constants $C>0$ and $0<\lambda \leq 1$ such that $\theta(\nu(x), \nu(y)) \leq$ $C|x-y|^{\lambda}$ for all $x, y \in \Gamma$, where $\theta(\nu(x), \nu(y))$ is the angle between $\nu(x)$ and $\nu(y)$, and $|x-y|$ denotes the Euclidean distance,

(L3) there exists a constant $d>0$ such that, for every $x \in \Gamma$, the subset $\Gamma \cap$ $B(x, d)$ is a graph over $T_{x} \Gamma$, where $B(x, d)$ denotes the closed ball of radius $d$ centered at $x$.

We refer to $\lambda$ and $d$ as a Lyapunov exponent and radius associated with $\Gamma$, and for any $x \in \Gamma$, refer to $B(x, d)$ as a Lyapunov ball at $x$. Notice that, if (L2) and (L3) hold for some values of $\lambda$ and $d$, then they also hold for all smaller values. We assume that values for $\lambda$ and $d$ are fixed once and for all, and for simplicity we assume from the outset that $\lambda=1$.

For any $x_{0} \in \Gamma$, we use $\Gamma_{x_{0}, d}$ to denote the portion of $\Gamma$ within the Lyapunov ball at $x_{0}$, and use $\Omega_{x_{0}, d}$ to denote the image of $\Gamma_{x_{0}, d}$ on $T_{x_{0}} \Gamma$ under projection parallel to $\nu\left(x_{0}\right)$. We refer to $\Gamma_{x_{0}, d}$ as the Lyapunov patch at $x_{0}$. Without loss of generality, we identify $\Omega_{x_{0}, d}$ with a subset of $\mathbb{R}^{2}$, and identify $x_{0}$ with the origin. We reserve 
the notation $T_{x_{0}} \Gamma$ to indicate the tangent plane considered as a subspace of $\mathbb{R}^{3}$. The Lyapunov condition (L3) implies that the map

$$
y=\psi_{x_{0}}(\xi), \quad y \in \Gamma_{x_{0}, d}, \quad \xi \in \Omega_{x_{0}, d},
$$

defined by projection parallel to $\nu\left(x_{0}\right)$, is a bijection. We refer to $y=\psi_{x_{0}}(\xi)$ with inverse $\xi=\psi_{x_{0}}^{-1}(y)$ as a local Cartesian parameterization of $\Gamma$ at $x_{0}$. When there is no cause for confusion, we abbreviate $\psi_{x_{0}}(\xi)$ by $y_{x_{0}}(\xi)$, and abbreviate $\psi_{x_{0}}^{-1}(y)$ by $\xi_{x_{0}}(y)$. For any $x_{0} \in \Gamma$ and $y \in \Gamma_{x_{0}, d}$, the local Cartesian coordinates $\xi_{x_{0}}(y)$ are uniquely defined up to the choice of orthonormal basis $\left\{t_{x_{0}, 1}, t_{x_{0}, 2}\right\}$ in $T_{x_{0}} \Gamma$. Specifically, we have

$$
\xi_{i}=\psi_{x_{0}, i}^{-1}(y)=\left(y-x_{0}\right) \cdot t_{x_{0}, i}, \quad i=1,2 .
$$

Throughout our analysis, we will also have need to consider a local polar parameterization of $\Gamma$ at $x_{0}$ of the form

$$
y=\psi_{x_{0}}^{\text {polar }}(\rho, \widehat{\xi})=\psi_{x_{0}}(\varpi(\rho, \widehat{\xi})), \quad y \in \Gamma_{x_{0}, d}, \quad(\rho, \widehat{\xi}) \in \Omega_{x_{0}, d}^{\text {polar }},
$$

where $\Omega_{x_{0}, d}^{\text {polar }}$ is a subset of $\mathbb{R}_{+} \times S$. Here $\mathbb{R}_{+}$denotes the non-negative reals and $S$ denotes the unit circle. Notice that the map $\xi=\varpi(\rho, \widehat{\xi})=\rho \widehat{\xi}$ is one-to-one for all $\xi \neq 0$, with inverse $(\rho, \widehat{\xi})=\varpi^{-1}(\xi)=(|\xi|, \xi /|\xi|)$, and is many-to-one at $\xi=0$, with $0=\varpi(0, \widehat{\xi})$ for all $\hat{\xi} \in S$. For any $x_{0} \in \Gamma$ and $y \in \Gamma_{x_{0}, d}$ with $y \neq x_{0}$ the map in (3) is invertible. Specifically, for any choice of orthonormal basis $\left\{t_{x_{0}, 1}, t_{x_{0}, 2}\right\}$ in $T_{x_{0}} \Gamma$, we have

$$
(\rho, \widehat{\xi})=\left[\psi_{x_{0}}^{\text {polar }}\right]^{-1}(y) \quad \text { where } \quad \rho=\left|y-x_{0}\right|, \widehat{\xi}_{i}=\frac{\left(y-x_{0}\right)_{i}}{\left|y-x_{0}\right|}, i=1,2 .
$$

We use $C^{m}(U, V)$ to denote the space of $m$-times continuously differentiable functions from $U \subset \mathbb{R}^{n}$ into $V \subset \mathbb{R}^{k}$, and $C^{m, 1}(U, V)$ to denote the subspace of functions whose derivatives up to order $m$ are Lipschitz. When $U$ is not an open set or the closure of an open set in $\mathbb{R}^{n}$, we interpret $C^{m}(U, V)$ to mean functions possessing an $m$-times continuously differentiable extension to an open set or the closure of an open set which contains $U$. For instance, when $U$ is a Lyapunov patch on $\Gamma$, we may consider extensions that are constant in the normal direction. We say that $\Gamma$ is of class $C^{m}$, and use the notation $\Gamma \in C^{m}$, if $\psi_{x_{0}} \in C^{m}\left(\Omega_{x_{0}, d}, \mathbb{R}^{3}\right)$ and $\psi_{x_{0}}^{-1} \in C^{m}\left(\Gamma_{x_{0}, d}, \mathbb{R}^{2}\right)$ for every $x_{0} \in \Gamma$. Similarly, we say that $\Gamma$ is of class $C^{m, 1}$, and use the notation $\Gamma \in C^{m, 1}$, if $\psi_{x_{0}} \in C^{m, 1}\left(\Omega_{x_{0}, d}, \mathbb{R}^{3}\right)$ and $\psi_{x_{0}}^{-1} \in C^{m, 1}\left(\Gamma_{x_{0}, d}, \mathbb{R}^{2}\right)$ for every $x_{0} \in \Gamma$, and additionally the Lipschitz constant for each derivative is uniform in $x_{0}$.

To any $f \in C^{1}\left(\Gamma, \mathbb{R}^{k}\right)$ we associate a surface derivative $D f \in C^{0}\left(\Gamma, \mathbb{R}^{k \times 3}\right)$. Specifically, for any point $y \in \Gamma$, we define $D f(y) \in \mathbb{R}^{k \times 3}$ as the usual derivative matrix at $y$ of an extension of $f$, where the extension is constant in the normal direction to $\Gamma$. When $f \in C^{1}\left(\Gamma, \mathbb{R}^{k}\right)$ is the restriction of some given $g \in C^{1}\left(\mathbb{R}^{3}, \mathbb{R}^{k}\right)$, the matrix $D f(y)$ is equivalent to the composition of the usual derivative matrix of $g$ with the projection matrix for orthogonal projection onto $T_{y} \Gamma$. In the case when $g$ is constant in the normal direction, the projection matrix can be replaced by the identity matrix and the two descriptions coincide. Notice that, for any curve $y(\tau) \in \Gamma$, we have $\frac{d}{d \tau} f(y(\tau))=D f(y(\tau)) \frac{d}{d \tau} y(\tau)$.

Given a function of the form $f(x, y)$ with $x, y \in \Gamma$, we use the notation $f_{x}(y)$ and $f_{y}(x)$ to denote the functions obtained by fixing the values of $x$ and $y$, respectively. 
Similarly, we use the notation $D_{x} f(x, y)$ and $D_{y} f(x, y)$ to denote the surface derivatives of $f(x, y)$ with respect to $x$ and $y$, respectively, where the other variable is held fixed. Hence $D_{x} f(x, y)=D f_{y}(x)$ and $D_{y} f(x, y)=D f_{x}(y)$. Higher-order surface derivatives for a function $f(y)$ are defined in a natural way. For instance, if we identify $\mathbb{R}^{k \times 3}$ with $\mathbb{R}^{3 k}$ and $D f \in C^{1}\left(\Gamma, \mathbb{R}^{3 k}\right)$, then $D^{2} f=D(D f) \in C^{0}\left(\Gamma, \mathbb{R}^{3 k \times 3}\right)$ is defined exactly as above. We denote the diagonal subset of $\Gamma \times \Gamma$ by $\Upsilon=\{(x, y) \in$ $\Gamma \times \Gamma \mid y=x\}$. Moreover, for any $\delta>0$ we also define an open $\delta$-neighborhood of the diagonal by $\Upsilon_{\delta}=\{(x, y) \in \Gamma \times \Gamma|| y-x \mid<\delta\}$. Throughout our developments, we use $|\cdot|$ to denote a Euclidean norm or the measure of a surface, as determined by the context.

2.2. Problem statement. Given a Lyapunov surface $\Gamma$ in three-dimensional space $\mathbb{R}^{3}$, we consider the problem of finding a function $\varphi \in C^{0}\left(\Gamma, \mathbb{R}^{k}\right)$ that satisfies

$$
c \varphi-\mathcal{A} \varphi=f,
$$

where $c \neq 0$ is a given constant, $f \in C^{0}\left(\Gamma, \mathbb{R}^{k}\right)$ is a given function, and $\mathcal{A}$ : $C^{0}\left(\Gamma, \mathbb{R}^{k}\right) \rightarrow C^{0}\left(\Gamma, \mathbb{R}^{k}\right)$ is a given integral operator of the form

$$
\mathcal{A} \varphi=\mathcal{G} \varphi+\mathcal{H} \varphi
$$

where

$$
(\mathcal{G} \varphi)(x)=\int_{\Gamma} G(x, y) \varphi(y) d A_{y}, \quad(\mathcal{H} \varphi)(x)=\int_{\Gamma} H(x, y) \varphi(y) d A_{y} .
$$

In the above, $d A_{y}$ denotes an infinitesimal area element at $y, G \in C^{0}\left(\Gamma \times \Gamma, \mathbb{R}^{k \times k}\right)$ is a continuous kernel, and $H \in C^{0}\left(\Gamma \times \Gamma \backslash \Upsilon, \mathbb{R}^{k \times k}\right)$ is a weakly singular kernel, which is unbounded along the diagonal subset $\Upsilon$. The above equation is typically considered with $k=1$ or 3 . The first case corresponds to a scalar-valued problem in three-dimensional space, as would arise in applications to electrostatics, and the second case corresponds to a vector-valued problem in three-dimensional space, as would arise in applications to elastostatics and hydrodynamics.

Our study of (5) will rely on various assumptions about the surface $\Gamma$ and the kernels $G$ and $H$. Specifically, we assume:

(A0) $\Gamma \in C^{m+1,1}$ for some $m \geq 0$, with Lyapunov radius $d>0$.

(A1) $G \in C^{m, 1}\left(\Gamma \times \Gamma, \mathbb{R}^{k \times k}\right)$.

(A2) $H \in C^{m, 1}\left(\Gamma \times \Gamma \backslash \Upsilon_{\delta}, \mathbb{R}^{k \times k}\right)$ for every $0<\delta \leq d$. Moreover, $H$ can be decomposed as

$$
H(x, y)=u(x, y) /|x-y|^{2-\mu},
$$

for some function $u \in C^{0}\left(\Gamma \times \Gamma \backslash \Upsilon, \mathbb{R}^{k \times k}\right)$ and exponent $0<\mu \leq 1$. Furthermore, on $\Gamma \times \Gamma \backslash \Upsilon$ the component functions $u_{i j}(1 \leq i, j \leq k)$ satisfy, for some positive constant $C$,

$$
\begin{array}{ll}
\left|u_{i j}(x, y)\right| \leq C, & \\
\left|D_{x}^{s} u_{i j}(x, y)\right| \leq C|y-x|^{-s}, & 1 \leq s \leq m+1, \\
\left|D_{y}^{s} u_{i j}(x, y)\right| \leq C|y-x|^{-s}, & 1 \leq s \leq m .
\end{array}
$$


(A3) When $m \geq 1$ we additionally assume that $\mu=1$ and that $u$ has the properties

$$
\begin{array}{lll}
\text { (i) } & u_{x_{0}}^{\text {polar }} \in C^{0,1}\left(\Omega_{x_{0}, d}^{\text {polar }}, \mathbb{R}^{k \times k}\right), & \forall x_{0} \in \Gamma, \\
\text { (ii) } & u_{x_{0}}^{\text {polar }}(0,-\widehat{\xi})=u_{x_{0}}^{\text {polar }}(0, \widehat{\xi}), & \forall \widehat{\xi} \in S, \\
\text { (iii) } & u_{x_{0}}^{\Delta} \in C^{0,1}\left(\Gamma_{x_{0}, d}, \mathbb{R}^{k \times k}\right), & \forall x_{0} \in \Gamma,
\end{array}
$$

where Lipschitz constants are uniform in $x_{0}$ and $u_{x_{0}}^{\text {polar }}=u_{x_{0}} \circ \psi_{x_{0}}^{\text {polar }}$ and

$$
u_{x_{0}}^{\Delta}(y)= \begin{cases}{\left.\left[u_{x_{0}}^{\text {polar }}(\rho, \widehat{\xi})-u_{x_{0}}^{\text {polar }}(0, \widehat{\xi})\right]\right|_{(\rho, \widehat{\xi})=\left[\psi_{x_{0}}^{\text {polar }}\right]^{-1}(y)},} & y \neq x_{0}, \\ 0, & y=x_{0} .\end{cases}
$$

Assumption (A0) states that $\Gamma$ must be at least of class $C^{1,1}$, which implies that $\Gamma$ is differentiable, with a curvature that is defined almost everywhere and bounded. Assumption (A1) states that the regularity of $G$ may be one order less than that of $\Gamma$, which will be convenient for our analysis. Assumption (A2) essentially states that $H$ is a weakly singular kernel, with regularity properties consistent with those of $G$. While the parameter $\mu$ could be identified with the Lyapunov exponent $\lambda$, we keep these parameters distinct for generality; indeed, various technical results presented later hold for arbitrary $0<\mu \leq 1$ and may be of independent interest. Assumption (A3) outlines additional regularity conditions on $H$ in the case when $\Gamma$ is at least of class $C^{2,1}$. The most specific condition is (A3)(ii), which states that the function $u_{x_{0}}^{\text {polar }}$ is an even function on $S$ at $\rho=0$. As we will see, this implies that certain local moments of $H$ will vanish. This condition will be important in our analysis of numerical methods and is related to the classic Tricomi condition that arises in the study of singular integral operators [47]. This condition is satisfied by weakly singular kernels $H$ arising in different applications, for example, the classic double-layer kernel in three-dimensional electrostatics, and incompressible elastostatics and hydrodynamics. We remark that condition (A3)(ii) is required for only the lowest-order method studied herein corresponding to a local polynomial correction of degree zero; it is not required for the higher-order methods.

2.3. Solvability theorem. The following result establishes the solvability of the boundary integral equation in (5). Its proof follows from the classic Fredholm Theorems for compact operators and is omitted for brevity; see, for example, 31, Chapter 4]. The compactness of $\mathcal{A}=\mathcal{G}+\mathcal{H}$ will be established in Lemma 4.5.

Theorem 2.1. Under conditions (A0)-(A2) the operator $\mathcal{A}$ on $C^{0}\left(\Gamma, \mathbb{R}^{k}\right)$ is compact. Hence, provided $c$ is not an associated eigenvalue, there exists a unique solution $\varphi \in C^{0}\left(\Gamma, \mathbb{R}^{k}\right)$ for any closed, bounded Lyapunov surface $\Gamma \in C^{1,1}$ and boundary data $f \in C^{0}\left(\Gamma, \mathbb{R}^{k}\right)$.

Thus, under mild conditions, the boundary integral equation in (5) has a unique solution $\varphi$ for any data $f$. Moreover, because the operator $c I-\mathcal{A}$ can be shown to have a bounded inverse, it follows that $\varphi$ depends continuously on $f$. The regularity of the solution $\varphi$ is determined by that of the surface $\Gamma$ and data $f$, and properties of the operator $\mathcal{A}$. The specific form of $\mathcal{A}$ given in (즈) arises in various applications as mentioned above. The component $\mathcal{H}$ may be interpreted as a double-layer potential. The component $\mathcal{G}$ may be zero, or may be interpreted as a range completion term necessary for the uniqueness of solutions. In the latter case, $\mathcal{G}$ may represent a potential due to a discrete or continuous distribution of sources 
located away from the surface. Such operators can be motivated beginning from a generalized boundary integral representation of a field by a linear combination of single- and double-layer potentials, and then moving the single-layer potential to an offset surface, and then possibly also shrinking the offset surface to a curve or point, or collection thereof. Various forms of such operators have been previously considered [20, 23, 25, 30, 33, 38, 40, 41].

\section{NySTRÖM APPROXIMATION}

3.1. Mesh, quadrature rule. We consider an arbitrary decomposition of $\Gamma$ into non-overlapping quadrature elements $\Gamma^{e}, e=1, \ldots, E$, each with area $\left|\Gamma^{e}\right|>0$. To any such decomposition we associate a size parameter $h=\max _{e}\left(\operatorname{diam}\left(\Gamma^{e}\right)\right)>$ 0 . For simplicity, we assume that the elements are either all quadrilateral or all triangular. In our analysis, we consider sequences of decompositions with increasing $E$, or equivalently, decreasing $h$. We will only consider sequences that satisfy a uniform refinement condition in the sense that the area of all elements is reduced at the same rate. Specifically, we assume

$$
C h^{2} \leq\left|\Gamma^{e}\right| \leq C^{\prime} h^{2}, \quad \forall e=1, \ldots, E, \quad E \geq E_{0} .
$$

Here $C, C^{\prime}$ and $E_{0}$ are positive constants whose values may change from one appearance to the next.

In each element $\Gamma^{e}$, we introduce quadrature nodes $x_{q}^{e}$ and weights $W_{q}^{e}>0$, $q=1, \ldots, Q$, where $Q$ is independent of $e$, such that

$$
\int_{\Gamma} f(x) d A_{x}=\sum_{e=1}^{E} \int_{\Gamma^{e}} f(x) d A_{x} \approx \sum_{e=1}^{E} \sum_{q=1}^{Q} f\left(x_{q}^{e}\right) W_{q}^{e} .
$$

We assume that the quadrature nodes and weights are defined by mapping each element $\Gamma^{e}$ to a standard, planar domain using a local parameterization, and applying a local quadrature rule in the standard domain. In this case, the Jacobian of the parameterization would be included in the weights $W_{q}^{e}$. We assume that the quadrature weights remain bounded and that the quadrature points remain distinct and in the element interiors. Specifically, for any sequence of decompositions that satisfy the uniform refinement condition, we assume

$$
\begin{gathered}
C h^{2} \leq \sum_{q=1}^{Q} W_{q}^{e} \leq C^{\prime} h^{2}, \quad \forall e=1, \ldots, E, \quad E \geq E_{0}, \\
C h \leq \operatorname{dist}\left(x_{q}^{e}, \partial \Gamma^{e}\right) \leq h, \quad \forall q=1, \ldots, Q, \quad e=1, \ldots, E, \quad E \geq E_{0}, \\
C h \leq \min _{(e, q) \neq\left(e^{\prime}, q^{\prime}\right)}\left|x_{q}^{e}-x_{q^{\prime}}^{e^{\prime}}\right| \leq h, \quad \forall q, q^{\prime}=1, \ldots, Q, \quad e, e^{\prime}=1, \ldots, E, \quad E \geq E_{0} .
\end{gathered}
$$

To quantify the error in a quadrature rule for a given function $f$ on a given surface $\Gamma$, we introduce the normalized local truncation errors

$$
\tau(e, f, h)=\frac{1}{\left|\Gamma^{e}\right|}\left|\int_{\Gamma^{e}} f(x) d A_{x}-\sum_{q=1}^{Q} f\left(x_{q}^{e}\right) W_{q}^{e}\right|, \quad e=1, \ldots, E .
$$


For sequences of decompositions that satisfy a uniform refinement condition, we require that the above truncation errors vanish uniformly in $e$ depending on properties of $f$. Specifically, we assume there exists an integer $\ell \geq 1$ such that

$$
\begin{gathered}
\max _{e} \tau(e, f, h) \rightarrow 0 \text { as } h \rightarrow 0 \text { for each } f \in C^{0}(\Gamma, \mathbb{R}), \\
\max _{e} \tau(e, f, h) \leq C h^{\ell} \text { for each } f \in C^{\ell-1,1}(\Gamma, \mathbb{R}) .
\end{gathered}
$$

In the above, the constant $C$ may depend on $f$, but is independent of $e$ and $h$, and the integer $\ell \geq 1$ is called the order of convergence of the quadrature rule.

For convenience, we will often replace the element and node indices $e=1, \ldots, E$ and $q=1, \ldots, Q$ with a single, general index $a=1, \ldots, n$, where $n=E Q$. We will use the multi- and single-index notation interchangeably with the understanding that there is a bijective map between the two.

3.2. Partition of unity functions. To each quadrature node $x_{a}$ in a decomposition of $\Gamma$ we associate nodal partition of unity functions $\zeta_{a}, \widehat{\zeta}_{a} \in C^{0}(\Gamma, \mathbb{R})$. We assume that these functions take values in the unit interval and are complementary so that their sum is equal to one. Moreover, we assume that $\zeta_{a}$ vanishes at least quadratically in a neighborhood of $x_{a}$, and that the support of $\widehat{\zeta}_{a}$ is bounded from above by a multiple of the mesh parameter $h$. Specifically, we assume

$$
\begin{gathered}
0 \leq \zeta_{a}(x), \widehat{\zeta}_{a}(x) \leq 1, \quad \zeta_{a}(x)+\widehat{\zeta}_{a}(x)=1, \quad \forall x \in \Gamma, \\
\left|\zeta_{a}(x)\right| \leq \frac{C\left|x-x_{a}\right|^{2}}{h^{2}}, \quad \operatorname{diam}\left(\operatorname{supp}\left(\widehat{\zeta}_{a}\right)\right) \leq C h, \quad \forall a=1, \ldots, n, \quad n \geq n_{0} .
\end{gathered}
$$

The functions $\zeta_{a}$ and $\widehat{\zeta}_{a}$ will play an important role in the family of numerical methods that we introduce and in the associated convergence proof. Specifically, these functions will help isolate the weak singularity of the kernel $H$ at each quadrature point and control the numerical error there.

3.3. Discretization of integral equation. Let a decomposition, quadrature rule, and nodal partition of unity functions for $\Gamma$ be given. For any function $f$, let $\mathcal{G}_{h}$ and $\mathcal{H}_{h}$ denote approximations to $\mathcal{G}$ and $\mathcal{H}$ of the form

$$
\left(\mathcal{G}_{h} f\right)(x)=\sum_{b=1}^{n} G_{b}(x) f\left(x_{b}\right), \quad\left(\mathcal{H}_{h} f\right)(x)=\sum_{b=1}^{n} H_{b}(x) f\left(x_{b}\right),
$$

where $G_{b}$ and $H_{b}$ are functions to be specified. Moreover, in view of (5), let $\varphi_{h}$ denote an approximation to $\varphi$ defined by

$$
c \varphi_{h}-\mathcal{A}_{h} \varphi_{h}=f \quad \text { where } \quad \mathcal{A}_{h}=\mathcal{G}_{h}+\mathcal{H}_{h} .
$$

The equation for $\varphi_{h}$ can be reduced to an $n \times n$ linear system for the nodal values $\varphi_{h}\left(x_{a}\right)$. Indeed, from (15) and (16) we get, for each $a=1, \ldots, n$,

$$
c \varphi_{h}\left(x_{a}\right)-\sum_{b=1}^{n} G_{b}\left(x_{a}\right) \varphi_{h}\left(x_{b}\right)-\sum_{b=1}^{n} H_{b}\left(x_{a}\right) \varphi_{h}\left(x_{b}\right)=f\left(x_{a}\right) .
$$

Notice that, for every solution of the discrete system (17), we obtain a solution of the continuous system (16), namely

$$
\varphi_{h}(x)=\frac{1}{c}\left[f(x)+\sum_{b=1}^{n} G_{b}(x) \varphi_{h}\left(x_{b}\right)+\sum_{b=1}^{n} H_{b}(x) \varphi_{h}\left(x_{b}\right)\right] .
$$


Moreover, the converse is also true; every solution of the continuous system provides a solution of the discrete system by restriction to the nodes.

The method is completed by specifying the functions $G_{b}(x)$ and $H_{b}(x)$. In view of (7), (13) and (15), we define $G_{b}(x)=G\left(x, x_{b}\right) W_{b}$. However, due to the weakly singular nature of the kernel function $H(x, y)$, a similar definition cannot be made for $H_{b}(x)$, because $H\left(x, x_{b}\right)$ is not continuous or even defined when $x=x_{b}$. Instead, for any integer $p \geq 0$ we define

$$
H_{b}(x)=\zeta_{b}(x) H\left(x, x_{b}\right) W_{b}+\widehat{\zeta}_{b}(x) R_{x}\left(x_{b}\right),
$$

where $R_{x}\left(x_{b}\right)$ is a local polynomial of degree $p$ at $x$, evaluated at quadrature point $x_{b}$. By a local polynomial at $x$ we mean a polynomial in any system of rectangular coordinates in the tangent plane with origin at $x$; see (21) below. For a Lyapunov surface $\Gamma$, such polynomials are well-defined in a neighborhood of each point $x$, and there is a uniform bound on the size of this neighborhood; indeed, they are welldefined in the Lyapunov patch $\Gamma_{x, d}$. The unknown coefficients in $R_{x}$ are defined by enforcing the local moment conditions

$$
\left(\mathcal{H}_{h} \eta_{x} f_{x}\right)(x)=\left(\mathcal{H} \eta_{x} f_{x}\right)(x) \text {, for all local polynomials } f_{x} \text { up to degree } p .
$$

Here $\eta_{x} \in C^{m, 1}(\Gamma,[0,1])$ is any given cut-off function which is identically one in a fixed neighborhood of $x$, for example, $\Gamma_{x, d / 4}$, and which is identically zero outside some fixed neighborhood of $x$, for example, $\Gamma_{x, d / 2}$. The local polynomials $R_{x}$ and $f_{x}$ can be described as floating since they are defined in a tangent plane that depends on $x$.

The basic structural form of $H_{b}$ is similar to $G_{b}$, but with a correction in a neighborhood of $x_{b}$. By construction, for all $x$ outside a neighborhood of $x_{b}$, we have $\zeta_{b}=1$ and $\widehat{\zeta}_{b}=0$, so that $H_{b}$ is defined by the quadrature rule applied to $H$. On the other hand, as $x$ approaches $x_{b}$, we have $\zeta_{b} \rightarrow 0$ and $\widehat{\zeta}_{b} \rightarrow 1$, so that $H_{b}$ is determined by the local polynomial $R_{x}$. The value of $R_{x}$ at a node $x_{b}$ can be interpreted as a generalized quadrature weight as would arise in a product integration method. There are various freedoms in the choice of $\eta_{x}$ used in the moment conditions. For the lowest-order method with $p=0$, the local polynomial $R_{x}$ reduces to a constant polynomial which can be extended to the entire surface, and the cut-off function is unnecessary and can be taken as unity. However, for higher-order methods with $p \geq 1$, the local polynomial $R_{x}$ in general cannot be extended to the entire surface, and a non-trivial cut-off function as described above is necessary. Moreover, a different cut-off function could be used for each different coefficient in $R_{x}$. For convenience, we employ a single cut-off function $\eta_{x}$ for all the moment conditions in our analysis below.

The moment conditions in (20) lead to a linear system of equations for the unknown coefficients of the local polynomial $R_{x}$, which can be solved for any given point $x$. Specifically, for any given $x \in \Gamma$, the local polynomial $R_{x}: \Gamma_{x, d} \rightarrow \mathbb{R}^{k \times k}$ of degree $p \geq 0$ has the form

$$
R_{x}(z)=\sum_{|\alpha| \leq p} C_{x, \alpha} \xi_{x}^{\alpha}(z)
$$

where $\alpha=\left(\alpha_{1}, \alpha_{2}\right)$ is a bi-index with non-negative integer components, $|\alpha|=$ $\alpha_{1}+\alpha_{2}$ denotes its associated degree, and the sum extends over all bi-indices $\alpha$ of degree up to $p$. In the above, $C_{x, \alpha} \in \mathbb{R}^{k \times k}$ are the coefficients of the polynomial, and $\xi_{x}^{\alpha} \in \mathbb{R}$ denote monomials in the local Cartesian coordinates $\xi_{x}=\left(\xi_{x, 1}, \xi_{x, 2}\right)$ 
such that $\xi_{x}^{\alpha}=\xi_{x, 1}^{\alpha_{1}} \xi_{x, 2}^{\alpha_{2}}$. Substituting (21) into (19), and using the standard basis for the local polynomials $f_{x}$, we find that the moment conditions in (20) lead to a linear system for the coefficients $C_{x, \alpha}$, namely

$$
\sum_{|\alpha| \leq p} M_{x, \alpha}^{\bar{\alpha}} C_{x, \alpha}=\Delta_{x}^{\bar{\alpha}}, \quad|\bar{\alpha}| \leq p,
$$

where $\alpha$ and $\bar{\alpha}$ are bi-indices, and $M_{x, \alpha}^{\bar{\alpha}} \in \mathbb{R}$ and $\Delta_{x}^{\bar{\alpha}} \in \mathbb{R}^{k \times k}$ are defined as

$$
\begin{gathered}
M_{x, \alpha}^{\bar{\alpha}}=\sum_{b=1}^{n} \widehat{\zeta}_{b}(x) \eta_{x}\left(x_{b}\right) \xi_{x}^{\alpha}\left(x_{b}\right) \xi_{x}^{\bar{\alpha}}\left(x_{b}\right), \\
\Delta_{x}^{\bar{\alpha}}=\int_{\Gamma} H(x, y) \eta_{x}(y) \xi_{x}^{\bar{\alpha}}(y) d A_{y}-\sum_{b=1}^{n} \zeta_{b}(x) H\left(x, x_{b}\right) \eta_{x}\left(x_{b}\right) \xi_{x}^{\bar{\alpha}}\left(x_{b}\right) W_{b} .
\end{gathered}
$$

A straightforward count in (22) shows that the number of $k \times k$ unknowns and the number of $k \times k$ equations are the same and equal to $(p+1)(p+2) / 2$. For instance, in the case $p=0$, we have a single $k \times k$ unknown $C_{x, 0}$ and a single $k \times k$ equation $M_{x, 0}^{0} C_{x, 0}=\Delta_{x}^{0}$, where the zero subscripts and superscripts denote biindices whose components are zero. Notice that the definition of the system involves various integral moments of the weakly singular kernel $H$. These moments can be evaluated numerically using techniques such as Duffy [16] or local polar coordinate 11, 12, 48 transformations. In the case when $p=0$ and the cut-off function is taken as unity, the required moment is known analytically in many applications. However, in the case when $p \geq 1$ and the cut-off function is non-trivial, the required moments must generally be obtained numerically.

For any given point $x \in \Gamma$, the solvability of the linear system in (22) for the coefficients $C_{x, \alpha}$ of the local polynomial $R_{x}$ depends on the support of the nodal functions $\widehat{\zeta}_{b}$. Specifically, let $J_{x}=\left\{b \mid \widehat{\zeta}_{b}(x)>0\right\}$ and consider sufficiently refined surface decompositions such that $\eta_{x}\left(x_{b}\right)=1$ for all $b \in J_{x}$. For any local scalarvalued polynomial $f_{x}$, let $F_{x}$ denote the vector of size $(p+1)(p+2) / 2$ of polynomial coefficients, and let $M_{x}$ denote the corresponding square, symmetric coefficient matrix of size $(p+1)(p+2) / 2$ associated with the equations in (22). Then by direct computation we find

$$
F_{x} \cdot M_{x} F_{x}=\sum_{b=1}^{n} \widehat{\zeta}_{b}(x)\left[f_{x}\left(x_{b}\right)\right]^{2} \eta_{x}\left(x_{b}\right)=\sum_{b \in J_{x}} \widehat{\zeta}_{b}(x)\left[f_{x}\left(x_{b}\right)\right]^{2} \geq 0 .
$$

From this we can deduce sufficient conditions for the positive-definiteness of $M_{x}$ and hence the unique solvability of (22). Specifically, for each $x \in \Gamma$, it is sufficient that $J_{x}$ be non-empty, and that the only polynomial of degree $p$ which satisfies $f_{x}\left(x_{b}\right)=$ 0 for all $b \in J_{x}$ be the zero polynomial. In view of the number of polynomial coefficients, this condition implies that $J_{x}$ must contain at least $(p+1)(p+2) / 2$ quadrature nodes for each $x \in \Gamma$. When $x$ is itself a quadrature node, this implies that the support of each function $\widehat{\zeta}_{b}$ must contain at least as many nodes. Hence the support of the functions $\widehat{\zeta}_{b}$ determines the solvability of (22). In our analysis, we will assume that $M_{x}$ has a uniformly bounded inverse, namely

$$
\left|M_{x}^{-1}\right| \leq C \quad \forall x \in \Gamma, \quad n \geq n_{0} .
$$

A straightforward choice of functions $\widehat{\zeta}_{b}$ which satisfy conditions (A7) and (A8) in the case $p=0$ is described below; see also 36. 
The implementation of the numerical method is centered upon (17), (18) and (22). For each quadrature node $x_{a}$, the linear system (22) is solved to obtain the local polynomials $R_{x_{a}}$, which are then used in the linear system (17) to obtain the nodal values $\varphi_{h}\left(x_{a}\right)$. Once these nodal values are determined, they can be extended to a continuous function. Specifically, given any point $x$, the linear system in (22) can be solved to obtain the local polynomial $R_{x}$, which can then be used in the interpolation equation (18) to obtain the value of $\varphi_{h}(x)$. Notice that, in general, the construction of the local polynomials $R_{x_{a}}$ and $R_{x}$ requires the evaluation of local moments of the weakly singular kernel $H$, and the evaluation of local Cartesian coordinates $\xi_{x_{a}}$ and $\xi_{x}$. As will be shown later, the values of $R_{x_{a}}$ and $R_{x}$ are independent of the choice of orthonormal basis associated with $\xi_{x_{a}}$ and $\xi_{x}$.

3.4. Illustrative example. Here we illustrate the form of the nodal equations (17) in the lowest-order case with $p=0$. In this case, the equations take a particularly simple form and the evaluation of local Cartesian coordinates is not necessary, and the evaluation of weakly singular integrals is typically not necessary.

We begin by describing partition of unity functions $\zeta_{a}$ and $\widehat{\zeta}_{a}$ which satisfy conditions (A7) and (A8). Consider an auxiliary decomposition of $\Gamma$ into Voronoi cells, where each cell contains a single quadrature point $x_{a}$. Each cell can be mapped to a unit circle, with $x_{a}$ mapped to the center. A simple quadratic function $z=\left(x^{2}+y^{2}\right) / 2$ in the unit circle can be mapped back to the cell as the central part of $\zeta_{a}$. We can then introduce an offset boundary which is displaced outward from the cell boundary by a distance of $\epsilon / 2$, where $\epsilon=\min _{a \neq b}\left|x_{a}-x_{b}\right|$. The mapped quadratic function, which by design has the value $1 / 2$ on the cell boundary, can be extended to achieve a value of 1 on the offset boundary, and then further extended to the rest of $\Gamma$ with the constant value 1 . Notice that the functions $\zeta_{a}$ and $\widehat{\zeta}_{a}$ so constructed have the convenient nodal property that $\zeta_{a}\left(x_{b}\right)=1-\delta_{a b}$ and $\widehat{\zeta}_{a}\left(x_{b}\right)=\delta_{a b}$. Moreover, the supports of $\widehat{\zeta}_{a}$ overlap on the entire surface and we have $\sum_{a} \widehat{\zeta}_{a}(x) \geq 1 / 2$ for all $x \in \Gamma$.

For the method with $p=0$, the local polynomial $R_{x}$ reduces to a constant polynomial $R_{x}(z) \equiv C_{x, 0}$, and the cut-off function $\eta_{x}$ can be taken as unity. The linear system in (22) reduces to the single equation $M_{x, 0}^{0} C_{x, 0}=\Delta_{x}^{0}$, which implies

$$
C_{x, 0}=\frac{\int_{\Gamma} H(x, y) d A_{y}-\sum_{b=1}^{n} \zeta_{b}(x) H\left(x, x_{b}\right) W_{b}}{\sum_{b=1}^{n} \widehat{\zeta}_{b}(x)} .
$$

This solution is well-defined and bounded for any sequence of decompositions by properties of the nodal functions $\zeta_{a}$ and $\widehat{\zeta}_{a}$ and the kernel function $H$. In various applications, the weakly singular integral in the above expression is known analytically and hence numerical evaluation is not necessary.

The nodal equations in (17) take a particularly simple form. Indeed, because our choice of the nodal partition of unity functions $\zeta_{a}$ and $\widehat{\zeta}_{a}$ has the property that $\zeta_{a}\left(x_{b}\right)=1-\delta_{a b}$ and $\widehat{\zeta}_{a}\left(x_{b}\right) \overline{\bar{n}} \delta_{a b}$, the equations become

$$
\begin{aligned}
\gamma\left(x_{a}\right) \varphi_{h}\left(x_{a}\right)-\sum_{b=1} G\left(x_{a}, x_{b}\right) \varphi_{h}\left(x_{b}\right) W_{b} \\
\quad-\sum_{\substack{b=1 \\
b \neq a}}^{n} H\left(x_{a}, x_{b}\right)\left[\varphi_{h}\left(x_{b}\right)-\varphi_{h}\left(x_{a}\right)\right] W_{b}=f\left(x_{a}\right),
\end{aligned}
$$

where $\gamma\left(x_{a}\right)=c I-\int_{\Gamma} H\left(x_{a}, y\right) d A_{y}$. This discrete system is similar to the classic singularity subtraction method discussed by various authors [4, 28]. The factor 
$\left[\varphi_{h}\left(x_{b}\right)-\varphi_{h}\left(x_{a}\right)\right]$ can be interpreted as cancelling the weak singularity in $H\left(x_{a}, x_{b}\right)$. Indeed, since the sum extends over $b \neq a$ only, the product $H\left(x_{a}, x_{b}\right)\left[\varphi_{h}\left(x_{b}\right)-\right.$ $\varphi_{h}\left(x_{a}\right)$ ] can be interpreted as vanishing when $b=a$. For methods with $p \geq 1$, a similar but higher-order cancellation can be interpreted to occur. Once the nodal values of $\varphi_{h}$ are determined, they can be extended to a continuous function using the interpolation equation in (18). Notice that the nodal values of $\varphi_{h}$ can be computed without explicit knowledge of the partition of unity functions. Various numerical examples with this method are given in [36.

3.5. Solvability and convergence theorem. The following result establishes the solvability and convergence of the locally-corrected Nyström method defined in (15) - 23). We consider the method with a quadrature rule of arbitrary order $\ell \geq 1$, a local polynomial correction of arbitrary degree $p \geq 0$, and a surface with regularity index $m \geq 0$. In view of Theorem 2.1, we suppose that the constant $c \neq 0$ is not an associated eigenvalue of the operator $\mathcal{A}$, so that the given boundary integral equation has a unique solution $\varphi$. Below we use $C_{\varphi}$ to denote a constant depending on $\varphi$.

Theorem 3.1. Under conditions (A0)-(A8), there exists a unique approximation $\varphi_{h} \in C^{0}\left(\Gamma, \mathbb{R}^{k}\right)$ for any closed, bounded Lyapunov surface $\Gamma \in C^{1,1}$ and boundary data $f \in C^{0}\left(\Gamma, \mathbb{R}^{k}\right)$ for all $h>0$ sufficiently small. Moreover, if $\varphi \in C^{m, 1}\left(\Gamma, \mathbb{R}^{k}\right)$ and $\Gamma \in C^{m+1,1}$, then as $h \rightarrow 0$,

$$
\forall \ell \geq 1, p \geq 0, m \geq 0
$$

$$
\begin{array}{ll}
\left\|\varphi_{h}-\varphi\right\| \leq C_{\varphi} h, & \forall \ell \geq 1, p=0, m \geq 1, \\
\left\|\varphi_{h}-\varphi\right\| \leq C_{\varphi} h^{\min (\ell, p, m)}, & \forall \ell \geq 1, p \geq 1, m \geq 1 .
\end{array}
$$

Thus, under suitable assumptions, the method defined by (15)-(23) is convergent in the usual maximum or $C^{0}$-norm. The rate of convergence depends on the order $\ell$ of the quadrature rule, the degree $p$ of the local polynomial correction, and the the regularity index $m$ of the exact solution $\varphi$ and the surface $\Gamma$. In the minimal regularity case with $m=0$, there is no lower bound on the rate, and in the higher regularity case with $m \geq 1$, the rate is at least linear. The rate of convergence is independent of the order of the quadrature rule in the case when $p=0$, which corresponds to the lowest degree of correction. Higher rates of convergence are obtained when $p \geq 1$, which corresponds to higher degrees of correction. Notice that the rates of convergence stated above are lower bounds; they could possibly be higher in certain circumstances, for example, in smooth problems with periodicity, for which some quadrature rules are known to have special properties [12,32. We remark that the method considered here is based on open quadrature rules as required by condition (A5), with positive weights. Straightforward rules of the Gauss-Legendre type are one example; various other possibilities for standard triangular and rectangular domains can be found in 46].

As a special case, Theorem 3.1 with $p=0$ establishes the convergence of a method similar to the classic singularity subtraction method considered previously by various authors $[4,28$. The two methods lead to apparently identical discrete systems for the nodal approximations, but differ in how the nodal approximations are interpolated over the surface. Convergence results for this method appear to be not well-known. The results derived here make crucial use of the structure of 
the nodal functions $H_{b}(x)$ defined in (19), the moment conditions defined in (20), and various properties of the nodal partition of unity functions $\zeta_{b}$ and $\widehat{\zeta}_{b}$. Such ingredients appear to have not been considered in previous studies of the classic method. We remark that the linear convergence result for $p=0$ is delicate and relies on a Tricomi-like property of the potential $\mathcal{H}$ implied by condition (A3)(ii). The results for $p \geq 1$ are qualitatively different and rely mainly on the regularizing effect of the local polynomial correction. Specifically, the linear convergence result in part (ii) requires condition (A3)(ii) whereas the convergence results in parts (i) and (iii) do not.

\section{Proof}

In this section we provide a proof of Theorem 3.1 We use the same notation and conventions as in previous sections. Specifically, we use $C, C^{\prime}, C^{\prime \prime}$ and so on, to denote generic positive constants whose value may change from one appearance to the next, use $|\cdot|$ to denote a Euclidean norm or the measure of a surface, as determined by the context, and use $\|\cdot\|$ to denote the usual maximum norm on $C^{0}\left(\Gamma, \mathbb{R}^{k}\right)$.

4.1. Collective compactness. We first outline a result, based on the theory of collectively compact operators 3 , which plays a fundamental role in the analysis of Nyström methods [7, 19,31]. Let $\mathcal{A}$ be a linear, compact operator on $C^{0}\left(\Gamma, \mathbb{R}^{k}\right)$ as given in (6), and let $\mathcal{A}_{n}\left(n \geq n_{0}\right)$ be a sequence of linear, finite-rank operators on $C^{0}\left(\Gamma, \mathbb{R}^{k}\right)$ as given in (16), where for convenience we consider subscripts $n \rightarrow \infty$ in place of $h_{n} \rightarrow 0$. Consider the following conditions:

(C1) For each $v \in C^{0}\left(\Gamma, \mathbb{R}^{k}\right),\left(\mathcal{A}_{n} v\right)(x) \rightarrow(\mathcal{A} v)(x)$ uniformly in $x \in \Gamma$.

(C2) $\left|\left(\mathcal{A}_{n} v\right)(x)\right| \leq C$ for all $x \in \Gamma$ and $v \in C^{0}\left(\Gamma, \mathbb{R}^{k}\right)$ with $\|v\| \leq 1$.

(C3) For every $\epsilon>0$ there exists $\delta>0$ and $N \geq n_{0}$ such that $\mid\left(\mathcal{A}_{n} v\right)(x)-$ $\left(\mathcal{A}_{n} v\right)(y) \mid<\epsilon$ for all $x, y \in \Gamma$ with $|x-y|<\delta, v \in C^{0}\left(\Gamma, \mathbb{R}^{k}\right)$ with $\|v\| \leq 1$, and $n \geq N$.

Condition $(\mathrm{C} 1)$ states that $\mathcal{A}_{n}$ converges to $\mathcal{A}$ pointwise in $C^{0}\left(\Gamma, \mathbb{R}^{k}\right)$. Conditions (C2) and (C3) state, respectively, that $S=\left\{\mathcal{A}_{n} v \mid n \geq n_{0},\|v\| \leq 1\right\}$ is an equibounded and equicontinuous subset of $C^{0}\left(\Gamma, \mathbb{R}^{k}\right)$. These two conditions imply, by the Arzela-Ascoli Theorem, that $S$ is relatively compact; by definition, the sequence $\mathcal{A}_{n}$ is then called collectively compact. For such sequences the following well-known result holds; see, for example, [7, Chapter 4] or [31, Chapter 10].

Theorem 4.1. Let $\mathcal{A}$ and $\mathcal{A}_{n}\left(n \geq n_{0}\right)$ be linear operators on $C^{0}\left(\Gamma, \mathbb{R}^{k}\right)$, where $\mathcal{A}$ is compact and $\mathcal{A}_{n}$ are finite-rank, and assume that (C1)-(C3) hold. If $c \varphi-\mathcal{A} \varphi=f$ is uniquely solvable for $\varphi$, then there exist constants $C>0$ and $N \geq n_{0}$ such that $c \varphi_{n}-\mathcal{A}_{n} \varphi_{n}=f$ is uniquely solvable for $\varphi_{n}$, and moreover,

$$
\left\|\varphi_{n}-\varphi\right\| \leq C\left\|\mathcal{A}_{n} \varphi-\mathcal{A} \varphi\right\|, \quad \forall n \geq N .
$$

In what follows, we establish conditions (C1)-(C3) for the operator $\mathcal{A}_{n}=\mathcal{G}_{n}+\mathcal{H}_{n}$ defined in (16). We concentrate on $\mathcal{H}_{n}$ since the result for $\mathcal{G}_{n}$ is straightforward by continuity of its kernel. Once $(\mathrm{C} 1)-(\mathrm{C} 3)$ are established, the result in Theorem 3.1 will follow from a bound on $\left\|\mathcal{A}_{n} \varphi-\mathcal{A} \varphi\right\|$ under regularity assumptions on $\Gamma$ and $\varphi$. 
4.2. Lemmata. We begin with a collection of useful results regarding the weakly singular kernel $H$. Parts (i) and (ii) below show that the integral operator defined by $H$ indeed maps $C^{0}\left(\Gamma, \mathbb{R}^{k}\right)$ into itself, and is equibounded and equicontinuous on bounded subsets, hence compact. Part (iii) is an important inequality that will be used in the sequel.

Lemma 4.2. Let $\Gamma$ satisfy (A0) with Lyapunov radius $d>0$ and let $H$ satisfy (A2) with exponent $0<\mu \leq 1$. Then:

(i) $\int_{\Gamma}|H(x, y)| d A_{y} \leq C$ for all $x \in \Gamma$.

(ii) For every $\epsilon>0$ there exists $\delta>0$ such that $\int_{\Gamma}\left|H\left(x_{*}, y\right)-H\left(x_{0}, y\right)\right| d A_{y} \leq \epsilon$ for all $x_{0}, x_{*} \in \Gamma$ with $\left|x_{0}-x_{*}\right| \leq \delta$.

(iii) $\left|H\left(x_{*}, y\right)-H\left(x_{0}, y\right)\right| \leq C\left|x_{*}-x_{0}\right| /\left|y-x_{0}\right|^{3-\mu}$ for all $x_{0}, x_{*}, y \in \Gamma$ with $0<\left|x_{*}-x_{0}\right| \leq d / 3$ and $2\left|x_{*}-x_{0}\right| \leq\left|y-x_{0}\right| \leq d$.

Proof. Parts (i) and (ii) are classic results for weakly singular integrals; see, for example, 23, Chapters 1,2], where the proof of (ii) relies on (iii). For brevity, we illustrate only (iii). To begin, we consider the form of $H$ in (8) and use the triangle inequality to get

$$
\begin{aligned}
\left|H\left(x_{*}, y\right)-H\left(x_{0}, y\right)\right| & =\left|\frac{u\left(x_{*}, y\right)}{\left|y-x_{*}\right|^{2-\mu}}-\frac{u\left(x_{0}, y\right)}{\left|y-x_{0}\right|^{2-\mu}}\right| \\
& \leq \frac{\left|u\left(x_{*}, y\right)-u\left(x_{0}, y\right)\right|}{\left|y-x_{*}\right|^{2-\mu}}+\left|\frac{u\left(x_{0}, y\right)}{\left|y-x_{*}\right|^{2-\mu}}-\frac{u\left(x_{0}, y\right)}{\left|y-x_{0}\right|^{2-\mu}}\right| .
\end{aligned}
$$

For the first term in (28), we consider the local Cartesian coordinate map $\psi_{x_{0}}$ in the Lyapunov patch $\Gamma_{x_{0}, d}$, and the curve $x(\tau)=\psi_{x_{0}}\left(\tau \xi_{*}\right), 0 \leq \tau \leq 1$, from $x_{0}=\psi_{x_{0}}(0)$ to $x_{*}=\psi_{x_{0}}\left(\xi_{*}\right)$. Using this curve, together with (10) and the relation $d x / d \tau=\left(\partial \psi_{x_{0}} / \partial \xi\right) \xi_{*}$, and the facts that $\left|\partial \psi_{x_{0}} / \partial \xi\right| \leq C$ and $\left|\xi_{*}\right| \leq C\left|x_{*}-x_{0}\right|$, which follow from the Lipschitz properties of $\psi_{x_{0}}$ and $\psi_{x_{0}}^{-1}$, we get

$$
\left|u\left(x_{*}, y\right)-u\left(x_{0}, y\right)\right|=\left|\int_{0}^{1} D_{x} u(x(\tau), y) \frac{d}{d \tau} x(\tau) d \tau\right| \leq \int_{0}^{1} \frac{C\left|x_{*}-x_{0}\right|}{|x(\tau)-y|} d \tau .
$$

From the definition of $x(\tau)$, we deduce that $\left|x(\tau)-x_{0}\right|$ is an increasing function, and hence $\left|x(\tau)-x_{0}\right| \leq\left|x_{*}-x_{0}\right|$ for all $0 \leq \tau \leq 1$. Moreover, by hypothesis, we have $0<\left|x_{*}-x_{0}\right| \leq\left|y-x_{0}\right| / 2$. These two results imply, with the help of the triangle inequality,

$$
\frac{1}{2} \leq \frac{|y-x(\tau)|}{\left|y-x_{0}\right|} \leq \frac{3}{2} \quad \forall \tau \in[0,1] \quad \text { and } \quad \frac{1}{2} \leq \frac{\left|y-x_{*}\right|}{\left|y-x_{0}\right|} \leq \frac{3}{2},
$$

and using (30) in (29), we find

$$
\left|u\left(x_{*}, y\right)-u\left(x_{0}, y\right)\right| \leq \frac{C\left|x_{*}-x_{0}\right|}{\left|y-x_{*}\right|} .
$$

For the second term in (28), we notice that $|u| \leq C$ by (9). Hence, using the notation $r=|y-x|$, we have

$$
\left|\frac{u\left(x_{0}, y\right)}{\left|y-x_{*}\right|^{2-\mu}}-\frac{u\left(x_{0}, y\right)}{\left|y-x_{0}\right|^{2-\mu}}\right| \leq C\left|\frac{1}{r_{*}^{2-\mu}}-\frac{1}{r_{0}^{2-\mu}}\right|=\frac{C\left|r_{0}^{2-\mu}-r_{*}^{2-\mu}\right|}{r_{0}^{2-\mu} r_{*}^{2-\mu}} .
$$

From (30) and the hypothesis $0<2\left|x_{*}-x_{0}\right| \leq\left|y-x_{0}\right| \leq d$ we notice that $0<r_{0} \leq d$ and $0<r_{0} \leq 2 r_{*} \leq 3 r_{0} \leq 3 d$. Moreover, since the function $r^{1-\mu}$ is monotonic, 
we have $\left|r_{0}^{2-\mu}-r_{*}^{2-\mu}\right|=\left|\int_{r_{0}}^{r_{*}} \frac{d}{d r}\left[r^{2-\mu}\right] d r\right| \leq(2-\mu) \max \left\{r_{0}^{1-\mu}, r_{*}^{1-\mu}\right\}\left|r_{*}-r_{0}\right|$. Combining these observations with (32), we find

$$
\left|\frac{u\left(x_{0}, y\right)}{\left|y-x_{*}\right|^{2-\mu}}-\frac{u\left(x_{0}, y\right)}{\left|y-x_{0}\right|^{2-\mu}}\right| \leq \frac{C\left|r_{*}-r_{0}\right|}{r_{0}^{3-\mu}} \leq \frac{C\left|x_{*}-x_{0}\right|}{\left|y-x_{0}\right|^{3-\mu}}
$$

where the last inequality follows from the definitions of $r_{0}$ and $r_{*}$, and the straightforward inequality $\left|r_{*}-r_{0}\right| \leq\left|x_{*}-x_{0}\right|$. Substituting (33) and (31) into (28), and again using (30) on the first term, we obtain the desired result.

The next result can be viewed, in part, as a discrete analog of Lemma 4.2. It will play a central role in establishing the collective compactness of the operators $\mathcal{H}_{n}$ associated with the numerical method. The defining elements of the method, which are the surface decomposition, quadrature rule, and nodal partition of unity functions, are denoted by the set $\left\{\Gamma^{e}, x_{q}^{e}, W_{q}^{e}, \zeta_{q}^{e}, \widehat{\zeta}_{q}^{e}\right\}$.

Lemma 4.3. Let $\Gamma$ satisfy (A0) with Lyapunov radius $d>0, H$ satisfy (A2) with exponent $0<\mu \leq 1$, and $\left\{\Gamma^{e}, x_{q}^{e}, W_{q}^{e}, \zeta_{q}^{e}, \widehat{\zeta}_{q}^{e}\right\}$ satisfy (A4)-(A7). Then:

(i) $\zeta_{b}(x) H\left(x, x_{b}\right)\left(:=0\right.$ at $\left.x=x_{b}\right)$ is continuous in $x \in \Gamma$ for each $b=1, \ldots, n$ and $n \geq n_{0}$.

(ii) $\sum_{b=1}^{n}\left|\zeta_{b}(x) H\left(x, x_{b}\right) W_{b}\right| \leq C$ for all $x \in \Gamma$ and $n \geq n_{0}$.

(iii) For every $\epsilon>0$ there exists $\delta>0$ such that $\sum_{b=1}^{n} \mid \zeta_{b}\left(x_{*}\right) H\left(x_{*}, x_{b}\right) W_{b}-$ $\zeta_{b}\left(x_{0}\right) H\left(x_{0}, x_{b}\right) W_{b} \mid \leq \epsilon$ for all $x_{0}, x_{*} \in \Gamma$ with $\left|x_{0}-x_{*}\right| \leq \delta$ and $n \geq n_{0}$.

Proof. For part (i), we notice that $\zeta_{b}(x)$ is continuous for all $x$, and $H\left(x, x_{b}\right)$ is continuous for all $x \neq x_{b}$. Hence, to establish the result, we need only verify that $\zeta_{b}(x) H\left(x, x_{b}\right) \rightarrow 0$ as $x \rightarrow x_{b}$. From (A2) and (A7), we get

$$
\left|\zeta_{b}(x) H\left(x, x_{b}\right)\right|=\frac{\left|\zeta_{b}(x) u\left(x, x_{b}\right)\right|}{\left|x-x_{b}\right|^{2-\mu}} \leq \frac{C\left|x-x_{b}\right|^{\mu}}{h^{2}}
$$

and the result follows since $0<\mu \leq 1$.

For part (ii), let $\delta \in(0, d / 3]$ and $\beta \geq 10$ be any given numbers and consider sufficiently refined surface decompositions with $E \geq E_{\beta, \delta}$, where $E_{\beta, \delta} \geq E_{0}$ is a given integer defined such that $0<\beta h \leq \delta / 2$ for all $E \geq E_{\beta, \delta}$. We consider an arbitrary $x=x_{0} \in \Gamma$ and consider collections of surface elements $\Gamma^{e}, e=1, \ldots, E$, defined by

$$
I_{x_{0}, \delta}=\left\{e \mid \Gamma^{e} \subset \Gamma_{x_{0}, \delta}\right\} \quad \text { and } \quad I_{x_{0}, \beta h}=\left\{e \mid \Gamma^{e} \subset \Gamma_{x_{0}, \beta h}\right\}
$$

and note that, by design, the sets $I_{x_{0}, \delta}, I_{x_{0}, \beta h} \subset I_{x_{0}, \delta}$ and $I_{x_{0}, \delta} \backslash I_{x_{0}, \beta h}$ are nonempty for all $E \geq E_{\beta, \delta}$. Moreover, we consider the decomposition

$$
\sum_{b=1}^{n} \zeta_{b}\left(x_{0}\right) H\left(x_{0}, x_{b}\right) W_{b}=\mathcal{S}_{1}\left(x_{0}\right)+\mathcal{S}_{2}\left(x_{0}\right)+\mathcal{S}_{3}\left(x_{0}\right)
$$


where

$$
\begin{aligned}
& \mathcal{S}_{1}\left(x_{0}\right)=\sum_{e \in I_{x_{0}, \beta h}} \sum_{q=1}^{Q} \zeta_{q}^{e}\left(x_{0}\right) H\left(x_{0}, x_{q}^{e}\right) W_{q}^{e}, \\
& \mathcal{S}_{2}\left(x_{0}\right)=\sum_{e \in I_{x_{0}, \delta} \backslash I_{x_{0}, \beta h}} \sum_{q=1}^{Q} \zeta_{q}^{e}\left(x_{0}\right) H\left(x_{0}, x_{q}^{e}\right) W_{q}^{e}, \\
& \mathcal{S}_{3}\left(x_{0}\right)=\sum_{e \notin I_{x_{0}, \delta}} \sum_{q=1}^{Q} \zeta_{q}^{e}\left(x_{0}\right) H\left(x_{0}, x_{q}^{e}\right) W_{q}^{e} .
\end{aligned}
$$

To establish the result, we show that each of the sums $\mathcal{S}_{1}, \mathcal{S}_{2}$ and $\mathcal{S}_{3}$ is uniformly bounded for all $E \geq E_{\beta, \delta}$ and $x_{0} \in \Gamma$. Boundedness for the finite interval $E \in$ $\left[E_{0}, E_{\beta, \delta}\right]$ is a straightforward consequence of part (i) and will be omitted for brevity.

For the term $\mathcal{S}_{1}$ in (36), we consider the local Cartesian coordinate map $\psi_{x_{0}}$ in the Lyapunov patch $\Gamma_{x_{0}, d}$. Since $\Gamma_{x_{0}, \beta h} \subset \Gamma_{x_{0}, d}$, and $\psi_{x_{0}}$ and $\psi_{x_{0}}^{-1}$ are Lipschitz, we have the area bound $\left|\Gamma_{x_{0}, \beta h}\right| \leq C h^{2}$. Moreover, from (A4) and the fact that $\left(\bigcup_{e \in I_{x_{0}, \beta h}} \Gamma^{e}\right) \subset \Gamma_{x_{0}, \beta h}$, we get $C h^{2}\left|I_{x_{0}, \beta h}\right| \leq\left|\Gamma_{x_{0}, \beta h}\right|$, where $\left|I_{x_{0}, \beta h}\right|$ denotes the number of elements in the index set $I_{x_{0}, \beta h}$. From these two observations, we deduce the uniform bound

$$
\left|I_{x_{0}, \beta h}\right| \leq C, \quad \forall E \geq E_{\beta, \delta}, \quad x_{0} \in \Gamma .
$$

From (37) we get, using (A2) and (A7),

$$
\left|\mathcal{S}_{1}\left(x_{0}\right)\right| \leq \sum_{e \in I_{x_{0}, \beta h}} \sum_{q=1}^{Q} \frac{C\left|x_{0}-x_{q}^{e}\right|^{2}}{h^{2}} \frac{C}{\left|x_{0}-x_{q}^{e}\right|^{2-\mu}} W_{q}^{e} .
$$

Since $x_{q}^{e} \in \Gamma^{e} \subset \Gamma_{x_{0}, \beta h}$ we have $\left|x_{0}-x_{q}^{e}\right| \leq \beta h$, and from (A5) we have $\sum_{q=1}^{Q} W_{q}^{e} \leq$ $C h^{2}$. Using these results together with (41) and (40), we find that

$$
\left|\mathcal{S}_{1}\left(x_{0}\right)\right| \leq C h^{\mu}, \quad \forall E \geq E_{\beta, \delta}, \quad x_{0} \in \Gamma,
$$

which establishes the result for $\mathcal{S}_{1}$ since $0<\mu \leq 1$. Indeed, the sum $\mathcal{S}_{1}\left(x_{0}\right) \rightarrow 0$ as $E \rightarrow \infty(h \rightarrow 0)$ uniformly in $x_{0} \in \Gamma$.

For the term $\mathcal{S}_{2}$ in (36), we notice first that $\Gamma_{x_{0},(\beta-1) h} \subset \Gamma_{x_{0}, \beta h}$ and that $\operatorname{dist}\left(\partial \Gamma_{x_{0},(\beta-1) h}, \partial \Gamma_{x_{0}, \beta h}\right) \geq h \geq \operatorname{diam}\left(\Gamma^{e}\right)$. Hence, if $\Gamma^{e} \cap \Gamma_{x_{0},(\beta-1) h} \neq \emptyset$, then $\Gamma^{e} \subset \Gamma_{x_{0}, \beta h}$. From this we deduce that $\Gamma^{e} \subset \Gamma_{x_{0}, \delta}$ and $\Gamma^{e} \cap \Gamma_{x_{0},(\beta-1) h}=\emptyset$ for all $e \in I_{x_{0}, \delta} \backslash I_{x_{0}, \beta h}$, which implies $0<(\beta-1) h<\left|x_{q}^{e}-x_{0}\right| \leq \delta$ for all $e \in I_{x_{0}, \delta} \backslash I_{x_{0}, \beta h}$ and $q=1, \ldots, Q$. Now, to each quadrature element $\Gamma^{e}$ with $e \in I_{x_{0}, \delta} \backslash I_{x_{0}, \beta h}$ we associate a distinguished node $q\left(x_{0}, e\right) \in\{1, \ldots, Q\}$, radius $\lambda\left(x_{0}, e\right) \in((\beta-1) h, \delta]$ and subset $\Gamma_{x_{0}, \text { in }}^{e} \subset \Gamma^{e}$ defined by

$$
\begin{gathered}
\min _{q=1, \ldots, Q}\left|x_{q}^{e}-x_{0}\right|=\left|x_{q\left(x_{0}, e\right)}^{e}-x_{0}\right|, \\
\lambda\left(x_{0}, e\right)=\left|x_{q\left(x_{0}, e\right)}^{e}-x_{0}\right|, \quad \Gamma_{x_{0}, \text { in }}^{e}=\Gamma^{e} \cap B\left(x_{0}, \lambda\left(x_{0}, e\right)\right) .
\end{gathered}
$$

From (A5) we get $C h \leq \operatorname{dist}\left(x_{q}^{e}, \partial \Gamma^{e}\right) \leq h$ for all $q=1, \ldots, Q$, and from this we deduce that the area of $\Gamma_{x_{0} \text {,in }}^{e}$ is bounded from below, namely $\left|\Gamma_{x_{0} \text {,in }}^{e}\right| \geq C h^{2}$. Combining this result with (A4), we find that

$$
C\left|\Gamma^{e}\right| \leq\left|\Gamma_{x_{0}, \text { in }}^{e}\right| \leq\left|\Gamma^{e}\right|, \quad \forall e \in I_{x_{0}, \delta} \backslash I_{x_{0}, \beta h}, \quad E \geq E_{\beta, \delta}
$$


From (38) we get, using (A2), (A4) and (A5), and the fact that $\left|\zeta_{q}^{e}\left(x_{0}\right)\right| \leq 1$, together with (43) 1 and (44),

$$
\begin{aligned}
\left|\mathcal{S}_{2}\left(x_{0}\right)\right| & \leq \sum_{e \in I_{x_{0}, \delta} \backslash I_{x_{0}, \beta h}} \sum_{q=1}^{Q} \frac{C}{\left|x_{0}-x_{q}^{e}\right|^{2-\mu}} W_{q}^{e}, \\
& \leq \sum_{e \in I_{x_{0}, \delta} \backslash I_{x_{0}, \beta h}} \frac{C}{\left|x_{0}-x_{q\left(x_{0}, e\right)}^{e}\right|^{2-\mu}} \sum_{q=1}^{Q} W_{q}^{e}, \\
& \leq \sum_{e \in I_{x_{0}, \delta} \backslash I_{x_{0}, \beta h}} \frac{C\left|\Gamma_{x_{0}, \text { in }}^{e}\right|}{\left|x_{0}-x_{q\left(x_{0}, e\right)}^{e}\right|^{2-\mu}} .
\end{aligned}
$$

Moreover, by definition of $\Gamma_{x_{0} \text {,in }}^{e}$ and the fact that $x_{q\left(x_{0}, e\right)}^{e} \in \partial B\left(x_{0}, \lambda\left(x_{0}, e\right)\right)$, we get

$$
\frac{1}{\left|x_{q\left(x_{0}, e\right)}^{e}-x_{0}\right|} \leq \frac{1}{\left|y-x_{0}\right|}, \quad \forall y \in \Gamma_{x_{0}, \text { in }}^{e} .
$$

Combining (46) with (45), and using the fact that $\Gamma_{x_{0}, \text { in }}^{e} \subset \Gamma^{e}$, we find

$$
\begin{aligned}
& \left|\mathcal{S}_{2}\left(x_{0}\right)\right| \leq \sum_{e \in I_{x_{0}, \delta \backslash I_{x_{0}}, \beta h}} \int_{\Gamma_{x_{0}, \text { in }}^{e}} \frac{C}{\left|y-x_{0}\right|^{2-\mu}} d A_{y}, \\
& \leq \sum_{e \in I_{x_{0}, \delta} \backslash I_{x_{0}, \beta h}} \int_{\Gamma^{e}} \frac{C}{\left|y-x_{0}\right|^{2-\mu}} d A_{y}, \\
& \leq \int_{\Gamma_{x_{0}, \delta}} \frac{C}{\left|y-x_{0}\right|^{2-\mu}} d A_{y} \leq C \delta^{\mu}, \quad \forall E \geq E_{\beta, \delta}, \quad x_{0} \in \Gamma,
\end{aligned}
$$

where the last inequality follows from a direct estimate of the integral using polar coordinates and the fact that the local coordinate maps $\psi_{x_{0}}$ and $\psi_{x_{0}}^{-1}$ are Lipschitz. Thus the result for $\mathcal{S}_{2}$ is established.

For the final term $\mathcal{S}_{3}$ in (36), we notice that $\Gamma^{e} \cap \Gamma_{x_{0}, \delta-h}=\emptyset$ for all $e \notin I_{x_{0}, \delta}$, which implies $0<\delta-h<\left|y-x_{0}\right|$ for all $y \in \Gamma^{e}, e \notin I_{x_{0}, \delta}$ and $E \geq E_{\beta, \delta}$. Using this fact in (39) we get, together with (A2), (A4) and (A5), and the fact that $\left|\zeta_{q}^{e}\left(x_{0}\right)\right| \leq 1$ and $\beta h \leq \delta / 2$,

$$
\left|\mathcal{S}_{3}\left(x_{0}\right)\right| \leq \sum_{e \notin I_{x_{0}, \delta}} \sum_{q=1}^{Q} \frac{C}{\left|x_{0}-x_{q}^{e}\right|^{2-\mu}} W_{q}^{e} \leq C \delta^{\mu-2}, \quad \forall E \geq E_{\beta, \delta}, \quad x_{0} \in \Gamma .
$$

Thus the result for $\mathcal{S}_{3}$ is established. The desired result stated in part (ii) follows from (48), (47), (42) and (36).

For part (iii), let $\delta \in(0, d / 3], \beta \geq 10$ and $E_{\beta, \delta} \geq E_{0}$ be given numbers defined as before, so that $0<\beta h \leq \delta / 2$ for all $E \geq E_{\beta, \delta}$. Moreover, in view of (A7), we suppose that $E_{\beta, \delta}$ is sufficiently large so that $\operatorname{diam}\left(\operatorname{supp}\left(\widehat{\zeta}_{b}\right)\right) \leq \delta / 2$ for all $b$. Since the result in part (iii) trivially holds when $x_{0}=x_{*}$, we consider, without loss of generality, arbitrary points $x_{0}, x_{*} \in \Gamma$ with $0<\left|x_{0}-x_{*}\right| \leq \delta$. Given such points, we consider the following collections of surface elements $\Gamma^{e}, e=1, \ldots, E$, defined in the same way as before:

$$
I_{x_{0}, \beta h} \subset I_{x_{0}, 2 \delta} \subset I_{x_{0}, d} \quad \text { and } \quad I_{x_{*}, \beta h} \subset I_{x_{*}, 3 \delta} \subset I_{x_{*}, d} .
$$


By design, each of the above sets is non-empty, as well as each of $I_{x_{0}, d} \backslash I_{x_{0}, 2 \delta}$, $I_{x_{0}, 2 \delta} \backslash I_{x_{0}, \beta h}, I_{x_{*}, d} \backslash I_{x_{*}, 3 \delta}$ and $I_{x_{*}, 3 \delta} \backslash I_{x_{*}, \beta h}$ for all $E \geq E_{\beta, \delta}$. Moreover, we have the inclusion $I_{x_{0}, 2 \delta} \subset I_{x_{*}, 3 \delta}$ for all $E \geq E_{\beta, \delta}$. Analogous to before, we consider the decomposition

$$
\sum_{b=1}^{n}\left[F_{b}\left(x_{0}\right)-F_{b}\left(x_{*}\right)\right]=\mathcal{F}_{1}\left(x_{0}, x_{*}\right)+\mathcal{F}_{2}\left(x_{0}, x_{*}\right)+\mathcal{F}_{3}\left(x_{0}, x_{*}\right),
$$

where $F_{b}(x)=\zeta_{b}(x) H\left(x, x_{b}\right) W_{b}$ and

$$
\begin{aligned}
& \mathcal{F}_{1}\left(x_{0}, x_{*}\right)=\sum_{e \in I_{x_{0}, 2 \delta}} \sum_{q=1}^{Q}\left[F_{q}^{e}\left(x_{0}\right)-F_{q}^{e}\left(x_{*}\right)\right], \\
& \mathcal{F}_{2}\left(x_{0}, x_{*}\right)=\sum_{e \in I_{x_{0}, d} \backslash I_{x_{0}, 2 \delta}} \sum_{q=1}^{Q}\left[F_{q}^{e}\left(x_{0}\right)-F_{q}^{e}\left(x_{*}\right)\right], \\
& \mathcal{F}_{3}\left(x_{0}, x_{*}\right)=\sum_{e \notin I_{x_{0}, d}} \sum_{q=1}^{Q}\left[F_{q}^{e}\left(x_{0}\right)-F_{q}^{e}\left(x_{*}\right)\right] .
\end{aligned}
$$

To establish the result, we show that, for every $\epsilon>0$, there exists a $\delta \in(0, d / 3]$ such that $\left|\mathcal{F}_{1}\right| \leq \epsilon,\left|\mathcal{F}_{2}\right| \leq \epsilon$ and $\left|\mathcal{F}_{3}\right| \leq \epsilon$ for all $0<\left|x_{0}-x_{*}\right| \leq \delta$ and $E \geq E_{\beta, \delta}$. Results for the finite interval $E \in\left[E_{0}, E_{\beta, \delta}\right]$ are a straightforward consequence of part (i) and will be omitted for brevity.

For the term $\mathcal{F}_{1}$ in (50), we use the inclusion $I_{x_{0}, 2 \delta} \subset I_{x_{*}, 3 \delta}$, and the inclusions $I_{x_{0}, \beta h} \subset I_{x_{0}, 2 \delta}$ and $I_{x_{*}, \beta h} \subset I_{x_{*}, 3 \delta}$, to obtain

$$
\begin{aligned}
\left|\mathcal{F}_{1}\left(x_{0}, x_{*}\right)\right| \leq & \sum_{e \in I_{x_{0}, 2 \delta}} \sum_{q=1}^{Q}\left|F_{q}^{e}\left(x_{0}\right)\right|+\sum_{e \in I_{x_{*}, 3 \delta}} \sum_{q=1}^{Q}\left|F_{q}^{e}\left(x_{*}\right)\right| \\
= & \left(\sum_{e \in I_{x_{0}, \beta h}}+\sum_{e \in I_{x_{0}, 2 \delta} \backslash I_{x_{0}, \beta h}}\right) \sum_{q=1}^{Q}\left|F_{q}^{e}\left(x_{0}\right)\right| \\
& +\left(\sum_{e \in I_{x_{*}, \beta h}}+\sum_{e \in I_{x_{*}, 3 \delta} \backslash I_{x_{*}, \beta h}}\right) \sum_{q=1}^{Q}\left|F_{q}^{e}\left(x_{*}\right)\right| .
\end{aligned}
$$

From the definition of $F_{q}^{e}$, and the same arguments in part (ii) that yielded (42) and (47), we find

$$
\left|\mathcal{F}_{1}\left(x_{0}, x_{*}\right)\right| \leq\left(C h^{\mu}+C \delta^{\mu}\right)+\left(C h^{\mu}+C \delta^{\mu}\right) \leq C \delta^{\mu}, \quad \forall E \geq E_{\beta, \delta},
$$

where $0<\mu \leq 1$ and the last inequality follows from the fact that $h \leq \delta /(2 \beta)$. Thus, for every $\epsilon>0$, we can choose $\delta \in(0, d / 3]$ sufficiently small to get the uniform bound

$$
\left|\mathcal{F}_{1}\left(x_{0}, x_{*}\right)\right| \leq \epsilon, \quad \forall E \geq E_{\beta, \delta}, \quad x_{0}, x_{*} \in \Gamma, \quad 0<\left|x_{0}-x_{*}\right| \leq \delta .
$$

For the term $\mathcal{F}_{2}$ in (50), we notice first that $\Gamma^{e} \subset \Gamma_{x_{0}, d}$ and $\Gamma^{e} \cap \Gamma_{x_{0}, 2 \delta-h}=\emptyset$ for all $e \in I_{x_{0}, d} \backslash I_{x_{0}, 2 \delta}$, which implies $\frac{39}{20} \delta \leq 2 \delta-h<\left|y-x_{0}\right| \leq d$ for all $y \in \Gamma^{e}$, $e \in I_{x_{0}, d} \backslash I_{x_{0}, 2 \delta}$ and $E \geq E_{\beta, \delta}$. Thus, by a slight generalization of Lemma4.2(iii), we have $\left|H\left(x_{*}, y\right)-H\left(x_{0}, y\right)\right| \leq C\left|x_{*}-x_{0}\right| /\left|y-x_{0}\right|^{3-\mu}$ for all $y \in \Gamma^{e}, e \in I_{x_{0}, d} \backslash I_{x_{0}, 2 \delta}$ and $E \geq E_{\beta, \delta}$. Furthermore, since $\operatorname{diam}\left(\operatorname{supp}\left(\widehat{\zeta}_{b}\right)\right) \leq \delta / 2$, we have $\zeta_{q}^{e}\left(x_{0}\right)=1$ 
and $\zeta_{q}^{e}\left(x_{*}\right)=1$ for all $x_{q}^{e} \in \Gamma^{e}, e \in I_{x_{0}, d} \backslash I_{x_{0}, 2 \delta}$ and $E \geq E_{\beta, \delta}$. Combining these observations with (52), and using the fact that $0<\left|x_{0}-x_{*}\right| \leq \delta$, we get

$$
\begin{aligned}
\left|\mathcal{F}_{2}\left(x_{0}, x_{*}\right)\right| & \leq \sum_{e \in I_{x_{0}, d} \backslash I_{x_{0}, 2 \delta}} \sum_{q=1}^{Q}\left|\zeta_{q}^{e}\left(x_{0}\right) H\left(x_{0}, x_{q}^{e}\right) W_{q}^{e}-\zeta_{q}^{e}\left(x_{*}\right) H\left(x_{*}, x_{q}^{e}\right) W_{q}^{e}\right|, \\
& \leq \sum_{e \in I_{x_{0}, d} \backslash I_{x_{0}, 2 \delta}} \sum_{q=1}^{Q}\left|H\left(x_{0}, x_{q}^{e}\right)-H\left(x_{*}, x_{q}^{e}\right)\right| W_{q}^{e}, \\
& \leq \sum_{e \in I_{x_{0}, d} \backslash I_{x_{0}, 2 \delta}} \sum_{q=1}^{Q} \frac{C \delta}{\left|x_{q}^{e}-x_{0}\right|^{3-\mu}} W_{q}^{e} .
\end{aligned}
$$

Using the same arguments as in (45)- (47) with analogous quantities $q\left(x_{0}, e\right), \lambda\left(x_{0}, e\right)$ and $\Gamma_{x_{0}, \text { in }}^{e}$, we obtain

$$
\begin{aligned}
\left|\mathcal{F}_{2}\left(x_{0}, x_{*}\right)\right| & \leq \sum_{e \in I_{x_{0}, d} \backslash I_{x_{0}, 2 \delta}} \int_{\Gamma^{e}} \frac{C \delta}{\left|y-x_{0}\right|^{3-\mu}} d A_{y}, \\
& \leq \int_{\Gamma_{x_{0}, d} \backslash \Gamma_{x_{0}, 2 \delta-h}} \frac{C \delta}{\left|y-x_{0}\right|^{3-\mu}} d A_{y}, \\
& \leq\left\{\begin{array}{lr}
C \delta+C \delta^{\mu}, & 0<\mu<1 \\
C \delta+C \delta|\ln \delta|, & \mu=1
\end{array}\right] \quad \forall E \geq E_{\beta, \delta},
\end{aligned}
$$

where the last inequality follows from a direct estimate of the integral using polar coordinates and the fact that the local coordinate maps $\psi_{x_{0}}$ and $\psi_{x_{0}}^{-1}$ are Lipschitz. Hence, for every $\epsilon>0$, we can choose $\delta \in(0, d / 3]$ sufficiently small to get the uniform bound

$$
\left|\mathcal{F}_{2}\left(x_{0}, x_{*}\right)\right| \leq \epsilon, \quad \forall E \geq E_{\beta, \delta}, \quad x_{0}, x_{*} \in \Gamma, \quad 0<\left|x_{0}-x_{*}\right| \leq \delta .
$$

For the last term $\mathcal{F}_{3}$ in (50), we notice that $\Gamma^{e} \cap \Gamma_{x_{0}, d-h}=\emptyset$ for all $e \notin I_{x_{0}, d}$. Using this, together with the fact that $h \leq \delta /(2 \beta) \leq d /(6 \beta)$, we find $\frac{59}{60} d \leq d-h<$ $\left|y-x_{0}\right|$ for all $y \in \Gamma^{e}, e \notin I_{x_{0}, d}$ and $E \geq E_{\beta, \delta}$. Moreover, since $0<\left|x_{0}-x_{*}\right| \leq$ $\delta \leq d / 3$, we deduce from the triangle inequality that $\frac{39}{60} d \leq\left|y-x_{*}\right|$ for all $y \in \Gamma^{e}$, $e \notin I_{x_{0}, d}$ and $E \geq E_{\beta, \delta}$. Furthermore, since diam(supp $\left.\left(\widehat{\zeta}_{b}\right)\right) \leq \delta / 2 \leq d / 6$, we have $\zeta_{q}^{e}\left(x_{0}\right)=1$ and $\zeta_{q}^{e}\left(x_{*}\right)=1$ for all $x_{q}^{e} \in \Gamma^{e}, e \notin I_{x_{0}, d}$ and $E \geq E_{\beta, \delta}$. Combining these observations with (53), and using the fact that $H(x, y)$ is Lipschitz on the set $|y-x| \geq 39 d / 60$ by (A2), and the fact that $0<\left|x_{0}-x_{*}\right| \leq \delta$, we get

$$
\begin{aligned}
\left|\mathcal{F}_{3}\left(x_{0}, x_{*}\right)\right| & \leq \sum_{e \notin I_{x_{0}, d}} \sum_{q=1}^{Q}\left|\zeta_{q}^{e}\left(x_{0}\right) H\left(x_{0}, x_{q}^{e}\right) W_{q}^{e}-\zeta_{q}^{e}\left(x_{*}\right) H\left(x_{*}, x_{q}^{e}\right) W_{q}^{e}\right|, \\
& \leq \sum_{e \notin I_{x_{0}, d}} \sum_{q=1}^{Q}\left|H\left(x_{0}, x_{q}^{e}\right)-H\left(x_{*}, x_{q}^{e}\right)\right| W_{q}^{e}, \\
& \leq \sum_{e \notin I_{x_{0}, d}} \sum_{q=1}^{Q} C\left|x_{0}-x_{*}\right| W_{q}^{e} \leq C|\Gamma|\left|x_{0}-x_{*}\right| \leq C \delta .
\end{aligned}
$$


Thus, for every $\epsilon>0$, we can choose $\delta \in(0, d / 3]$ sufficiently small to get the uniform bound

$$
\left|\mathcal{F}_{3}\left(x_{0}, x_{*}\right)\right| \leq \epsilon, \quad \forall E \geq E_{\beta, \delta}, \quad x_{0}, x_{*} \in \Gamma, \quad 0<\left|x_{0}-x_{*}\right| \leq \delta .
$$

The desired result stated in part (iii) follows from (61), (59), (56) and (50).

Our next result establishes some important properties of the local polynomial $R_{x}(z)$ of degree $p \geq 0$ with coefficients $C_{x, \alpha}$ defined in (21)-23). Part (i) below is straightforward and is included for completeness.

Lemma 4.4. Let $\Gamma$ satisfy (A0) with Lyapunov radius $d>0, H$ satisfy (A2) with exponent $0<\mu \leq 1$, and $\left\{\Gamma^{e}, x_{q}^{e}, W_{q}^{e}, \zeta_{q}^{e}, \widehat{\zeta}_{q}^{e}\right\}$ satisfy (A4)-(A8). Then:

(i) For each $x \in \Gamma$ and $n \geq n_{0}$, the local polynomial $R_{x}: \Gamma_{x, d} \rightarrow \mathbb{R}^{k \times k}$ is invariant to the choice of orthonormal basis in $T_{x} \Gamma$.

(ii) $R_{x}(z)$ is continuous in $x \in \Gamma$ and $z \in \Gamma_{x, d^{\prime}}$ for each $n \geq n_{0}$ and $d^{\prime} \in(0, d)$.

(iii) For every $\epsilon>0$ there exists $N>0$ such that $\left|R_{x}(z)\right| \leq \epsilon$ for all $x \in \Gamma$, $z \in \Gamma_{x, d}$ and $n \geq N$

Proof. The result in part (i) follows from a standard change-of-basis argument in $T_{x} \Gamma$, along with the uniqueness of solutions of (22) guaranteed by (A8).

For part (ii), let $n \geq n_{0}, d^{\prime} \in(0, d), x_{0} \in \Gamma$ and $z_{0} \in \Gamma_{x_{0}, d^{\prime}}$ be arbitrary, and consider any $0<\delta<\left(d-d^{\prime}\right) / 2$. Then, by the triangle inequality, we find

$$
z \in \Gamma_{x, d}, \quad \forall x \in \Gamma_{x_{0}, \delta}, \quad \forall z \in \Gamma_{z_{0}, \delta} .
$$

Moreover, let $u_{0} \in T_{x_{0}} \Gamma$ be any given unit vector, let $\nu(x)$ denote the outward unit normal to $\Gamma$ at $x$, and consider a basis for $T_{x} \Gamma$ defined by

$$
t_{1}(x)=\frac{u_{0}-\left(u_{0} \cdot \nu(x)\right) \nu(x)}{\left|u_{0}-\left(u_{0} \cdot \nu(x)\right) \nu(x)\right|}, \quad t_{2}(x)=\nu(x) \times t_{1}(x) .
$$

Then, for any $x \in \Gamma_{x_{0}, \delta}$ and $z \in \Gamma_{z_{0}, \delta}$, the local Cartesian coordinates for $z \in \Gamma_{x, d}$ are given by

$$
\xi_{x, 1}(z)=(z-x) \cdot t_{1}(x), \quad \xi_{x, 2}(z)=(z-x) \cdot t_{2}(x) .
$$

From the fact that these coordinates depend continuously on $x \in \Gamma_{x_{0}, \delta}$ and $z \in$ $\Gamma_{z_{0}, \delta}$, together with Lemmas 4.2 and 4.3 , we deduce that the coefficient matrix and data vector for the linear system in (22) depend continuously on $x \in \Gamma_{x_{0}, \delta}$. Furthermore, by (A8), the unique solution $C_{x, \alpha}$ of the system also depends continuously on $x \in \Gamma_{x_{0}, \delta}$. Combining these results with (21), we find that $R_{x}(z)$ depends continuously on $x \in \Gamma_{x_{0}, \delta}$ and $z \in \Gamma_{z_{0}, \delta}$ as required.

For part (iii), we notice that, by (A8) and the fact that $\left|\xi_{x}(z)\right| \leq d$ for all $x \in \Gamma$ and $z \in \Gamma_{x, d}$, it will be sufficient to show that, for every $\epsilon>0$, there exists an $N>0$ such that

$$
\left|\Delta_{x}^{\bar{\alpha}}\right| \leq \epsilon, \quad \forall n \geq N, \quad x \in \Gamma, \quad|\bar{\alpha}| \leq p .
$$

For brevity, we establish the above bound for $\Delta_{x}^{0}$ only, and note that a bound for $\Delta_{x}^{\bar{\alpha}}$ with $0<|\bar{\alpha}| \leq p$ follows by a similar argument. Using the same notation as in the proof of Lemma 4.3. let $\delta \in(0, d / 3], \beta \geq 10$ and $E_{\beta, \delta} \geq E_{0}$ be given numbers, where $E_{\beta, \delta}$ is sufficiently large such that $0<\beta h \leq \delta / 2$ and $\operatorname{diam}\left(\operatorname{supp}\left(\widehat{\zeta}_{b}\right)\right) \leq \delta / 2$ for all $b=1, \ldots, n$ and $E \geq E_{\beta, \delta}$. Moreover, for arbitrary $x=x_{0} \in \Gamma$, we consider 
as before the collections of surface elements $\Gamma^{e}, e=1, \ldots, E$, denoted by $I_{x_{0}, \beta h}$ and $I_{x_{0}, \delta}$. From (23), we consider the decomposition

$$
\begin{aligned}
\Delta_{x_{0}}^{0} & =\int_{\Gamma} H\left(x_{0}, y\right) \eta_{x_{0}}(y) d A_{y}-\sum_{b=1}^{n} \zeta_{b}\left(x_{0}\right) H\left(x_{0}, x_{b}\right) \eta_{x_{0}}\left(x_{b}\right) W_{b}, \\
& =\mathcal{D}_{1}\left(x_{0}\right)+\mathcal{D}_{2}\left(x_{0}\right),
\end{aligned}
$$

where

$$
\mathcal{D}_{1}\left(x_{0}\right)=\sum_{e \in I_{x_{0}, \delta}}\left[\int_{\Gamma^{e}} H\left(x_{0}, y\right) \eta_{x_{0}}(y) d A_{y}-\sum_{q=1}^{Q} \zeta_{q}^{e}\left(x_{0}\right) H\left(x_{0}, x_{q}^{e}\right) \eta_{x_{0}}\left(x_{q}^{e}\right) W_{q}^{e}\right],
$$

$$
\mathcal{D}_{2}\left(x_{0}\right)=\sum_{e \notin I_{x_{0}, \delta}}\left[\int_{\Gamma^{e}} H\left(x_{0}, y\right) \eta_{x_{0}}(y) d A_{y}-\sum_{q=1}^{Q} \zeta_{q}^{e}\left(x_{0}\right) H\left(x_{0}, x_{q}^{e}\right) \eta_{x_{0}}\left(x_{q}^{e}\right) W_{q}^{e}\right] .
$$

To establish the result, we show that, for every $\epsilon>0$, we can choose $E_{\beta, \delta} \geq E_{0}$ sufficiently large such that $\left|\mathcal{D}_{1}\right| \leq \epsilon$ and $\left|\mathcal{D}_{2}\right| \leq \epsilon$ for all $E \geq E_{\beta, \delta}$ and $x_{0} \in \Gamma$.

For the term $\mathcal{D}_{1}$ in (66), we use the fact that $\left|\eta_{x_{0}}\right| \leq 1$ to obtain

$$
\left|\mathcal{D}_{1}\left(x_{0}\right)\right| \leq \sum_{e \in I_{x_{0}, \delta}}\left[\int_{\Gamma^{e}}\left|H\left(x_{0}, y\right)\right| d A_{y}+\sum_{q=1}^{Q}\left|\zeta_{q}^{e}\left(x_{0}\right) H\left(x_{0}, x_{q}^{e}\right) W_{q}^{e}\right|\right] .
$$

Working with the first sum in (69), we have, using (A2),

$$
\sum_{e \in I_{x_{0}, \delta}} \int_{\Gamma^{e}}\left|H\left(x_{0}, y\right)\right| d A_{y} \leq \int_{\Gamma_{x_{0}, \delta}} \frac{C}{\left|y-x_{0}\right|^{2-\mu}} d A_{y} \leq C \delta^{\mu}, \quad \forall E \geq E_{\beta, \delta},
$$

where $0<\mu \leq 1$ and the last inequality follows from a direct estimate of the integral using polar coordinates and the fact that the local coordinate maps $\psi_{x_{0}}$ and $\psi_{x_{0}}^{-1}$ are Lipschitz. Working with the second sum in (69), we have, using the inclusion $I_{x_{0}, \beta h} \subset I_{x_{0}, \delta}$,

$$
\begin{aligned}
\sum_{e \in I_{x_{0}}, \delta} \sum_{q=1}^{Q}\left|\zeta_{q}^{e}\left(x_{0}\right) H\left(x_{0}, x_{q}^{e}\right) W_{q}^{e}\right| & \\
& =\left(\sum_{e \in I_{x_{0}, \beta h}}+\sum_{e \in I_{x_{0}, \delta} \backslash I_{x_{0}, \beta h}}\right) \sum_{q=1}^{Q}\left|\zeta_{q}^{e}\left(x_{0}\right) H\left(x_{0}, x_{q}^{e}\right) W_{q}^{e}\right| .
\end{aligned}
$$

By the same arguments used in the proof of Lemma 4.3(ii) that yielded (42) and (47), we find

$$
\sum_{e \in I_{x_{0}}, \delta} \sum_{q=1}^{Q}\left|\zeta_{q}^{e}\left(x_{0}\right) H\left(x_{0}, x_{q}^{e}\right) W_{q}^{e}\right| \leq\left(C h^{\mu}+C \delta^{\mu}\right) \leq C \delta^{\mu}, \quad \forall E \geq E_{\beta, \delta},
$$

where the last inequality follows from the fact that $h \leq \delta /(2 \beta)$. Thus, in view of (72), (70) and (69), for every $\epsilon>0$, we can choose a $\delta \in(0, d / 3]$ sufficiently small to get the uniform bound

$$
\left|\mathcal{D}_{1}\left(x_{0}\right)\right| \leq \epsilon, \quad \forall E \geq E_{\beta, \delta}, \quad x_{0} \in \Gamma .
$$


For the term $\mathcal{D}_{2}$ in (66), we notice that $\Gamma^{e} \cap \Gamma_{x_{0}, \delta-h}=\emptyset$ for all $e \notin I_{x_{0}, \delta}$. From this, along with the fact that $h \leq \delta /(2 \beta)$, we get $\frac{19}{20} \delta \leq \delta-h<\left|y-x_{0}\right|$ for all $y \in \Gamma^{e}, e \notin I_{x_{0}, \delta}$ and $E \geq E_{\beta, \delta}$. Furthermore, since diam( $\left.\operatorname{supp}\left(\widehat{\zeta}_{b}\right)\right) \leq \delta / 2$, we have $\zeta_{q}^{e}\left(x_{0}\right)=1$ for all $x_{q}^{e} \in \Gamma^{e}, e \notin I_{x_{0}, \delta}$ and $E \geq E_{\beta, \delta}$. Combining these observations with (68), and using the fact that $H(x, y)$ is class $C^{m, 1}$ on the set $|y-x| \geq 19 \delta / 20$ by (A2), and that $\eta_{x}(y)$ is class $C^{m, 1}$ for all $x$ and $y$, together with the quadrature error bound in (A6) for a rule of order $\ell$, we get

$$
\begin{aligned}
\left|\mathcal{D}_{2}\left(x_{0}\right)\right| & \leq \sum_{e \notin I_{x_{0}, \delta}}\left|\int_{\Gamma^{e}} H\left(x_{0}, y\right) \eta_{x_{0}}(y) d A_{y}-\sum_{q=1}^{Q} H\left(x_{0}, x_{q}^{e}\right) \eta_{x_{0}}\left(x_{q}^{e}\right) W_{q}^{e}\right| \\
& \leq \sum_{e \notin I_{x_{0}, \delta}} C_{\delta}\left|\Gamma^{e}\right| h^{\min (\ell, m+1)} \leq C_{\delta}|\Gamma| h^{\min (\ell, m+1)}, \quad \forall E \geq E_{\beta, \delta} .
\end{aligned}
$$

Here $C_{\delta}$ is a Lipschitz constant for the derivatives of the function $H(x, y) \eta_{x}(y)$ on the set $|y-x| \geq 19 \delta / 20$, and $\delta \in(0, d / 3]$ is fixed such that (73) holds. Thus, for any given $\epsilon>0$, we can choose $E_{\beta, \delta}$ sufficiently large (equivalently $h$ sufficiently small) to get the uniform bound

$$
\left|\mathcal{D}_{2}\left(x_{0}\right)\right| \leq \epsilon, \quad \forall E \geq E_{\beta, \delta}, \quad x_{0} \in \Gamma .
$$

The desired result for $\Delta_{x_{0}}^{0}$ follows from (75), (73) and (66).

The next result shows that the linear operators $\mathcal{A}$ and $\mathcal{A}_{n}, n \geq n_{0}$ defined in (6) and (16) satisfy the collective compactness conditions (C1)-(C3).

Lemma 4.5. Let $\Gamma$ satisfy (A0) with Lyapunov radius $d>0, G$ satisfy (A1), $H$ satisfy (A2) with exponent $0<\mu \leq 1$, and $\left\{\Gamma^{e}, x_{q}^{e}, W_{q}^{e}, \zeta_{q}^{e}, \widehat{\zeta}_{q}^{e}\right\}$ satisfy (A4)-(A8). Then:

(i) $\mathcal{A}$ is a compact operator on $C^{0}\left(\Gamma, \mathbb{R}^{k}\right)$.

(ii) $\mathcal{A}_{n}$ is a finite-rank (hence compact) operator on $C^{0}\left(\Gamma, \mathbb{R}^{k}\right)$ for each $n \geq n_{0}$.

(iii) $\mathcal{A}_{n}, n \geq n_{0}$ satisfies (C1)-(C3) and hence is a collectively compact sequence on $C^{0}\left(\Gamma, \mathbb{R}^{k}\right)$.

Proof. The result for $\mathcal{A}=\mathcal{G}+\mathcal{H}$ in part (i) is classic and follows from the continuity of $G$ and the properties of $H$ established in Lemma 4.2, see, for example, 31, Chapter 2]. The result for $\mathcal{A}_{n}=\mathcal{G}_{n}+\mathcal{H}_{n}$ in part (ii) is analogous and relies on Lemma 4.3. For brevity, we illustrate only (iii). Moreover, we show the result only for $\mathcal{H}_{n}$. The result for $\mathcal{G}_{n}$ is similar and more straightforward due to the continuity of $G$.

To establish (C1), let $v \in C^{0}\left(\Gamma, \mathbb{R}^{k}\right)$ be arbitrary. We seek to show that $\left|(\mathcal{H} v)\left(x_{0}\right)-\left(\mathcal{H}_{n} v\right)\left(x_{0}\right)\right| \rightarrow 0$ as $n \rightarrow \infty$ uniformly in $x_{0} \in \Gamma$, where $\mathcal{H}$ and $\mathcal{H}_{n}$ are as defined in (77) and (15)-(23). Using the same notation as in the proof of Lemma 4.3. let $\delta \in(0, d / 3], \beta \geq 10$ and $E_{\beta, \delta} \geq E_{0}$ be given numbers, where $E_{\beta, \delta}$ is sufficiently large such that $0<\beta h \leq \delta / 2$ and $\operatorname{diam}\left(\operatorname{supp}\left(\widehat{\zeta}_{b}\right)\right) \leq \delta / 2$ for all $b=1, \ldots, n$ and $E \geq E_{\beta, \delta}$. Moreover, for any given $x_{0} \in \Gamma$, consider as before the collections of surface elements $\Gamma^{e}, e=1, \ldots, E$, denoted by $I_{x_{0}, \beta h}$ and $I_{x_{0}, \delta}$, and consider the decomposition

$$
(\mathcal{H} v)\left(x_{0}\right)-\left(\mathcal{H}_{n} v\right)\left(x_{0}\right)=\mathcal{K}_{1}\left(x_{0}\right)+\mathcal{K}_{2}\left(x_{0}\right)-\mathcal{K}_{3}\left(x_{0}\right),
$$


where

$$
\begin{aligned}
\mathcal{K}_{1}\left(x_{0}\right) & =\sum_{e \in I_{x_{0}, \delta}}\left[\int_{\Gamma^{e}} H\left(x_{0}, y\right) v(y) d A_{y}-\sum_{q=1}^{Q} \zeta_{q}^{e}\left(x_{0}\right) H\left(x_{0}, x_{q}^{e}\right) v\left(x_{q}^{e}\right) W_{q}^{e}\right] \\
\mathcal{K}_{2}\left(x_{0}\right) & =\sum_{e \notin I_{x_{0}, \delta}}\left[\int_{\Gamma^{e}} H\left(x_{0}, y\right) v(y) d A_{y}-\sum_{q=1}^{Q} \zeta_{q}^{e}\left(x_{0}\right) H\left(x_{0}, x_{q}^{e}\right) v\left(x_{q}^{e}\right) W_{q}^{e}\right] \\
\mathcal{K}_{3}\left(x_{0}\right) & =\sum_{b=1}^{n} \widehat{\zeta}_{b}\left(x_{0}\right) R_{x_{0}}\left(x_{b}\right) v\left(x_{b}\right) .
\end{aligned}
$$

To establish the result, we show that, for every $\epsilon>0$, we can choose $E_{\beta, \delta} \geq E_{0}$ sufficiently large such that $\left|\mathcal{K}_{1}\right| \leq \epsilon,\left|\mathcal{K}_{2}\right| \leq \epsilon$ and $\left|\mathcal{K}_{3}\right| \leq \epsilon$ for all $E \geq E_{\beta, \delta}$ and $x_{0} \in \Gamma$.

For the term $\mathcal{K}_{1}$ in (76) , the same arguments used to establish (69) $-(72)$ in the proof of Lemma 4.4 can be applied to obtain

$$
\begin{aligned}
\left|\mathcal{K}_{1}\left(x_{0}\right)\right| & \leq \sum_{e \in I_{x_{0}, \delta}}\|v\|\left[\int_{\Gamma^{e}}\left|H\left(x_{0}, y\right)\right| d A_{y}+\sum_{q=1}^{Q}\left|\zeta_{q}^{e}\left(x_{0}\right) H\left(x_{0}, x_{q}^{e}\right) W_{q}^{e}\right|\right], \\
& \leq C\|v\| \delta^{\mu}, \quad \forall E \geq E_{\beta, \delta} .
\end{aligned}
$$

Thus, for every $\epsilon>0$, we can choose a $\delta \in(0, d / 3]$ sufficiently small to get the uniform bound

$$
\left|\mathcal{K}_{1}\left(x_{0}\right)\right| \leq \epsilon, \quad \forall E \geq E_{\beta, \delta}, \quad x_{0} \in \Gamma .
$$

For the term $\mathcal{K}_{2}$ in (76), the same arguments used to establish (74) in the proof of Lemma 4.4 can be applied to obtain

$$
\begin{aligned}
\left|\mathcal{K}_{2}\left(x_{0}\right)\right| & \leq \sum_{e \notin I_{x_{0}, \delta}}\left|\int_{\Gamma^{e}} H\left(x_{0}, y\right) v(y) d A_{y}-\sum_{q=1}^{Q} H\left(x_{0}, x_{q}^{e}\right) v\left(x_{q}^{e}\right) W_{q}^{e}\right| \\
& \leq \sum_{e \notin I_{x_{0}, \delta}}\left|\Gamma^{e}\right| \tau\left(e, f_{x_{0}}, h\right) \leq|\Gamma| \max _{e \notin I_{x_{0}}, \delta} \tau\left(e, f_{x_{0}}, h\right), \quad \forall E \geq E_{\beta, \delta} .
\end{aligned}
$$

Here $f_{x_{0}}(y)=H\left(x_{0}, y\right) v(y)$ is the function being integrated, $\tau\left(e, f_{x_{0}}, h\right)$ is the quadrature truncation error for this function as given in (A6), $e \notin I_{x_{0}, \delta}$ implies $\left|y-x_{0}\right| \geq 19 \delta / 20$, and $\delta \in(0, d / 3]$ is fixed such that (81) holds. Since $H\left(x_{0}, y\right)$ is class $C^{m, 1}$ in $x_{0}$ and $y$ by (A2) on the set $\left|y-x_{0}\right| \geq 19 \delta / 20$, it follows that the continuity properties of $f_{x_{0}}(y)$ in $y$ are uniform in $x_{0}$. Hence, by (A6), $\max _{e \notin I_{x_{0}, \delta}} \tau\left(e, f_{x_{0}}, h\right) \rightarrow 0$ as $h \rightarrow 0$ uniformly in $x_{0}$. From this we deduce that, for any given $\epsilon>0$, we can choose $E_{\beta, \delta}$ sufficiently large (equivalently $h$ sufficiently small) to get the uniform bound

$$
\left|\mathcal{K}_{2}\left(x_{0}\right)\right| \leq \epsilon, \quad \forall E \geq E_{\beta, \delta}, \quad x_{0} \in \Gamma .
$$

For the term $\mathcal{K}_{3}$ in (76), we consider the index set $J_{x_{0}}=\left\{b \mid \widehat{\zeta}_{b}\left(x_{0}\right)>0\right\}$. By (A7), we have $\operatorname{diam}\left(\operatorname{supp}\left(\widehat{\zeta}_{b}\right)\right) \leq C h$, and by (A5), we have $\left|x_{a}-x_{b}\right| \geq C h$ for any $a \neq b$. From this we deduce that the number of elements in $J_{x_{0}}$, denoted by $\left|J_{x_{0}}\right|$, must be bounded uniformly in $x_{0}$, namely

$$
\left|J_{x_{0}}\right| \leq C, \quad \forall E \geq E_{\beta, \delta}, \quad x_{0} \in \Gamma .
$$


From (779) we get, using the definition of $J_{x_{0}}$ and the fact that $0 \leq \widehat{\zeta}_{b} \leq 1$,

$$
\left|\mathcal{K}_{3}\left(x_{0}\right)\right| \leq \sum_{b=1}^{n}\left|\widehat{\zeta}_{b}\left(x_{0}\right) R_{x_{0}}\left(x_{b}\right) v\left(x_{b}\right)\right| \leq\|v\|\left|\sum_{b \in J_{x_{0}}}\right| R_{x_{0}}\left(x_{b}\right) \mid .
$$

Using (85) and (84), together with Lemma 4.4(iii), we find that, for any given $\epsilon>0$, we can choose $E_{\beta, \delta}$ sufficiently large to get the uniform bound

$$
\left|\mathcal{K}_{3}\left(x_{0}\right)\right| \leq \epsilon, \quad \forall E \geq E_{\beta, \delta}, \quad x_{0} \in \Gamma .
$$

The desired result follows from (86), (83), (81) and (76).

To establish (C2), we seek to show that $\left(\mathcal{H}_{n} v\right)\left(x_{0}\right)$ is uniformly bounded for all $n \geq n_{0}, x_{0} \in \Gamma$ and $v \in C^{0}\left(\Gamma, \mathbb{R}^{k}\right)$ with $\|v\| \leq 1$. By definition of $\mathcal{H}_{n}$, we have

$$
\left(\mathcal{H}_{n} v\right)\left(x_{0}\right)=\sum_{b=1}^{n}\left[\zeta_{b}\left(x_{0}\right) H\left(x_{0}, x_{b}\right) v\left(x_{b}\right) W_{b}+\widehat{\zeta}_{b}\left(x_{0}\right) R_{x_{0}}\left(x_{b}\right) v\left(x_{b}\right)\right],
$$

and from the definition of $J_{x_{0}}$ given above, and the fact that $0 \leq \widehat{\zeta}_{b} \leq 1$, we get

$$
\left|\left(\mathcal{H}_{n} v\right)\left(x_{0}\right)\right| \leq\|v\|\left|\sum_{b=1}^{n}\right| \zeta_{b}\left(x_{0}\right) H\left(x_{0}, x_{b}\right) W_{b}\left|+\|v\| \sum_{b \in J_{x_{0}}}\right| R_{x_{0}}\left(x_{b}\right) \mid .
$$

From this we deduce, using Lemmas 4.3(ii) and 4.4(iii), the uniform bound

$$
\left|\left(\mathcal{H}_{n} v\right)\left(x_{0}\right)\right| \leq C, \quad \forall n \geq n_{0}, \quad x_{0} \in \Gamma, \quad v \in C^{0}\left(\Gamma, \mathbb{R}^{k}\right), \quad\|v\| \leq 1 .
$$

To establish (C3), we seek to show that $\left(\mathcal{H}_{n} v\right)\left(x_{0}\right)$ is uniformly equicontinuous for all $n \geq n_{0}, x_{0} \in \Gamma$ and $v \in C^{0}\left(\Gamma, \mathbb{R}^{k}\right)$ with $\|v\| \leq 1$. For arbitrary $x_{0}, x_{*} \in \Gamma$, we have, by definition of $\mathcal{H}_{n}$,

$$
\begin{aligned}
& \left(\mathcal{H}_{n} v\right)\left(x_{*}\right)-\left(\mathcal{H}_{n} v\right)\left(x_{0}\right) \\
& =\sum_{b=1}^{n} \zeta_{b}\left(x_{*}\right) H\left(x_{*}, x_{b}\right) v\left(x_{b}\right) W_{b}-\sum_{b=1}^{n} \zeta_{b}\left(x_{0}\right) H\left(x_{0}, x_{b}\right) v\left(x_{b}\right) W_{b} \\
& \quad+\sum_{b=1}^{n} \widehat{\zeta}_{b}\left(x_{*}\right) R_{x_{*}}\left(x_{b}\right) v\left(x_{b}\right)-\sum_{b=1}^{n} \widehat{\zeta}_{b}\left(x_{0}\right) R_{x_{0}}\left(x_{b}\right) v\left(x_{b}\right) .
\end{aligned}
$$

Similar to before, we consider the decomposition

$$
\left(\mathcal{H}_{n} v\right)\left(x_{*}\right)-\left(\mathcal{H}_{n} v\right)\left(x_{0}\right)=\mathcal{Q}\left(x_{0}, x_{*}\right)+\mathcal{R}\left(x_{*}\right)-\mathcal{R}\left(x_{0}\right)
$$

where

$$
\begin{aligned}
& \mathcal{Q}\left(x_{0}, x_{*}\right)=\sum_{b=1}^{n}\left[\zeta_{b}\left(x_{*}\right) H\left(x_{*}, x_{b}\right) W_{b}-\zeta_{b}\left(x_{0}\right) H\left(x_{0}, x_{b}\right) W_{b}\right] v\left(x_{b}\right), \\
& \mathcal{R}\left(x_{*}\right)=\sum_{b=1}^{n} \widehat{\zeta}_{b}\left(x_{*}\right) R_{x_{*}}\left(x_{b}\right) v\left(x_{b}\right), \quad \mathcal{R}\left(x_{0}\right)=\sum_{b=1}^{n} \widehat{\zeta}_{b}\left(x_{0}\right) R_{x_{0}}\left(x_{b}\right) v\left(x_{b}\right) .
\end{aligned}
$$

To establish the result, we show that, for every $\epsilon>0$, we can choose a $\delta \in(0, d / 3]$ such that $|\mathcal{Q}| \leq \epsilon$ and $|\mathcal{R}| \leq \epsilon$ for all $x_{0}, x_{*} \in \Gamma$ with $\left|x_{0}-x_{*}\right|<\delta, v \in C^{0}\left(\Gamma, \mathbb{R}^{k}\right)$ with $\|v\| \leq 1$, and $n \geq n_{0}$. Notice that this result immediately follows from 
Lemma 4.3(iii), Lemma 4.4(iii) and (84), after increasing the value of $n_{0}$ if necessary, since

$$
\begin{gathered}
\left|\mathcal{Q}\left(x_{0}, x_{*}\right)\right| \leq\|v\| \sum_{b=1}^{n}\left|\zeta_{b}\left(x_{*}\right) H\left(x_{*}, x_{b}\right) W_{b}-\zeta_{b}\left(x_{0}\right) H\left(x_{0}, x_{b}\right) W_{b}\right|, \\
\left|\mathcal{R}\left(x_{*}\right)\right| \leq\|v\| \sum_{b \in J_{x_{*}}}\left|R_{x_{*}}\left(x_{b}\right)\right|, \quad\left|\mathcal{R}\left(x_{0}\right)\right| \leq\|v\| \sum_{b \in J_{x_{0}}}\left|R_{x_{0}}\left(x_{b}\right)\right| .
\end{gathered}
$$

The next result will be crucial in establishing the linear rate of convergence of the lowest-order method with $p=0$. We employ the notation from condition (A3). Moreover, for any given $x_{0} \in \Gamma$ and $\varphi \in C^{1,1}\left(\Gamma, \mathbb{R}^{k}\right)$, we introduce the function $U_{x_{0}, \varphi}: \Gamma_{x_{0}, d} \rightarrow \mathbb{R}^{k}$ defined as

$$
U_{x_{0}, \varphi}(y)= \begin{cases}H\left(x_{0}, y\right)\left[\varphi(y)-\varphi\left(x_{0}\right)\right]-u_{x_{0}}^{\text {polar }}(0, \widehat{\xi}(y)) D \varphi\left(x_{0}\right) T_{x_{0}}(y), & y \neq x_{0}, \\ 0, & y=x_{0} .\end{cases}
$$

Here $T_{x_{0}}(y) \in T_{x_{0}} \Gamma$ is the unit vector defined from the orthogonal projection of the chord $y-x_{0}$ onto $T_{x_{0}} \Gamma$, and $\widehat{\xi}(y) \in S$ is the angular polar coordinate associated with $y \neq x_{0}$. Specifically, we consider the functions $T_{x_{0}}: \Gamma_{x_{0}, d} \backslash\left\{x_{0}\right\} \rightarrow \mathbb{R}^{3}$ and $T_{x_{0}}^{\Delta}: \Gamma_{x_{0}, d} \rightarrow \mathbb{R}^{3}$ defined as

$$
T_{x_{0}}(y)=\frac{\left(y-x_{0}\right)-\left(\left(y-x_{0}\right) \cdot \nu_{0}\right) \nu_{0}}{\left|\left(y-x_{0}\right)-\left(\left(y-x_{0}\right) \cdot \nu_{0}\right) \nu_{0}\right|}, \quad T_{x_{0}}^{\Delta}(y)= \begin{cases}\frac{\left(y-x_{0}\right)}{\left|y-x_{0}\right|}-T_{x_{0}}(y), & y \neq x_{0} \\ 0, & y=x_{0}\end{cases}
$$

where $\nu_{0}=\nu\left(x_{0}\right)$ is the outward unit normal to $\Gamma$ at $x_{0}$. We remark that the result in part (ii)(b) below, and its discrete analog in part (ii)(c), are reminiscent of the classic Tricomi condition that arises in the study of singular integral operators [47.

Lemma 4.6. Let $\Gamma$ satisfy (A0) with Lyapunov radius $d>0$ and regularity index $m \geq 1$, $H$ satisfy (A2) and (A3) with exponent $\mu=1$, and $\left\{\Gamma^{e}, x_{q}^{e}, W_{q}^{e}\right\}$ satisfy (A4)-(A6). Then:

(i) For every $x_{0} \in \Gamma$ and $\varphi \in C^{1,1}\left(\Gamma, \mathbb{R}^{k}\right)$ we have $U_{x_{0}, \varphi} \in C^{0,1}\left(\Gamma_{x_{0}, d}, \mathbb{R}^{k}\right)$ with Lipschitz constant uniform in $x_{0}$. Hence $\left|U_{x_{0}, \varphi}(y)\right| \leq C_{\varphi}\left|y-x_{0}\right|$ for all $x_{0} \in \Gamma$ and $y \in \Gamma_{x_{0}, d}$.

(ii) For every $x_{0} \in \Gamma$ there exists a patch $\Gamma_{x_{0}}^{*} \subset \Gamma$ such that

(a) $\Gamma_{x_{0}, d / C} \subset \Gamma_{x_{0}}^{*} \subset \Gamma_{x_{0}, d}$,

(b) $\int_{\Gamma_{x_{0}}^{*}} u_{x_{0}}^{\text {polar }}(0, \widehat{\xi}(y)) D \varphi\left(x_{0}\right) T_{x_{0}}(y) d A_{y}=0$ for each $\varphi \in C^{1,1}\left(\Gamma, \mathbb{R}^{k}\right)$,

(c) $\left|\sum_{e \in I_{x_{0}}^{*}} \sum_{1 \leq q \leq Q}^{\prime} u_{x_{0}}^{\text {polar }}\left(0, \widehat{\xi}\left(x_{q}^{e}\right)\right) D \varphi\left(x_{0}\right) T_{x_{0}}\left(x_{q}^{e}\right) W_{q}^{e}\right| \leq C_{\varphi} h$ for all $n \geq$ $n_{0}$ and each $\varphi \in C^{1,1}\left(\Gamma, \mathbb{R}^{k}\right)$.

In the above, $C$ denotes a fixed constant, $C_{\varphi}$ denotes a constant depending on $\varphi$, the sum with a prime is over those $q$ such that $x_{q}^{e} \neq x_{0}$, and $I_{x_{0}}^{*}=\left\{e \mid \Gamma^{e} \subset \Gamma_{x_{0}}^{*}\right\}$.

Proof. For part (i), let $x_{0} \in \Gamma$ and $\varphi \in C^{1,1}\left(\Gamma, \mathbb{R}^{k}\right)$ be arbitrary. By working with local Cartesian coordinates in the Lyapunov patch $\Gamma_{x_{0}, d}$ and using Taylor's Theorem for Lipschitz functions, we find

$$
\varphi(y)=\varphi\left(x_{0}\right)+D \varphi\left(x_{0}\right)\left[y-x_{0}\right]+\mathcal{R}_{x_{0}}(y), \quad \forall y \in \Gamma_{x_{0}, d},
$$


where

$$
\begin{gathered}
\left|\mathcal{R}_{x_{0}}(y)\right| \leq C_{\varphi}\left|y-x_{0}\right|^{2} \\
\mathcal{R}_{x_{0}} \in C^{1,1}\left(\Gamma_{x_{0}, d}, \mathbb{R}^{k}\right), \quad \frac{\mathcal{R}_{x_{0}}}{\left|y-x_{0}\right|} \in C^{0,1}\left(\Gamma_{x_{0}, d}, \mathbb{R}^{k}\right) .
\end{gathered}
$$

Moreover, since $\varphi$ is globally Lipschitz on $\Gamma$, the Lipschitz constants for $\mathcal{R}_{x_{0}}$ and $\mathcal{R}_{x_{0}} /\left|y-x_{0}\right|$ are uniform in $x_{0} \in \Gamma$, where the latter function is defined to be zero when $y=x_{0}$.

To establish properties of the functions $T_{x_{0}}$ and $T_{x_{0}}^{\Delta}$, for any given $y \in \Gamma_{x_{0}, d}$ with $y \neq x_{0}$, we consider an arbitrary curve $\gamma(\tau) \in \Gamma_{x_{0}, d}$ such that $\gamma(0)=y$. Then, by direct calculation from their definitions, we find $\left|\frac{d}{d \tau} T_{x_{0}}(\gamma(\tau))\right|_{\tau=0} \mid \leq$ $C\left|\gamma^{\prime}(0)\right| /\left|y-x_{0}\right|$ and $\left|\frac{d}{d \tau} T_{x_{0}}^{\Delta}(\gamma(\tau))\right|_{\tau=0}|\leq C| \gamma^{\prime}(0) \mid$. From this, we deduce

$$
\begin{gathered}
\left|D T_{x_{0}}(y)\right| \leq \frac{C}{\left|y-x_{0}\right|}, \quad\left|D T_{x_{0}}^{\Delta}(y)\right| \leq C, \quad \forall y \in \Gamma_{x_{0}, d}, \quad y \neq x_{0}, \\
T_{x_{0}}^{\Delta} \in C^{0,1}\left(\Gamma_{x_{0}, d}, \mathbb{R}^{3}\right),
\end{gathered}
$$

where the last result above follows from the fact that $T_{x_{0}}^{\Delta}(y)$ has a uniformly bounded surface derivative for almost every $y \in \Gamma_{x_{0}, d}$. Notice that the Lipschitz constant for $T_{x_{0}}^{\Delta}$, equivalently the bound on its surface derivative, is uniform in $x_{0} \in \Gamma$. Moreover, by definition of $T_{x_{0}}$, and the Lipschitz property of $T_{x_{0}}^{\Delta}$, and the fact that $T_{x_{0}}^{\Delta}\left(x_{0}\right)=0$, we have the bounds

$$
\left|T_{x_{0}}(y)\right|=1, \quad\left|T_{x_{0}}^{\Delta}(y)\right| \leq C\left|y-x_{0}\right|, \quad \forall y \in \Gamma_{x_{0}, d}, \quad y \neq x_{0} .
$$

Proceeding with part (i), for any $y \in \Gamma_{x_{0}, d}$ with $y \neq x_{0}$, we use (99), (98) and (96), and (A2) with $\mu=1$, to write

$$
\begin{aligned}
& U_{x_{0}, \varphi}(y) \\
& \quad=H\left(x_{0}, y\right)\left[\varphi(y)-\varphi\left(x_{0}\right)\right]-u_{x_{0}}^{\text {polar }}(0, \widehat{\xi}(y)) D \varphi\left(x_{0}\right) T_{x_{0}}(y), \\
& \quad=u\left(x_{0}, y\right)\left[D \varphi\left(x_{0}\right) \frac{\left(y-x_{0}\right)}{\left|y-x_{0}\right|}+\frac{\mathcal{R}_{x_{0}}(y)}{\left|y-x_{0}\right|}\right]-u_{x_{0}}^{\text {polar }}(0, \widehat{\xi}(y)) D \varphi\left(x_{0}\right) T_{x_{0}}(y) .
\end{aligned}
$$

In terms of the functions $T_{x_{0}}^{\Delta}$ defined in (97) and $u_{x_{0}}^{\Delta}$ defined in (A3)(iii), this becomes

$$
\begin{aligned}
U_{x_{0}, \varphi}(y)=u\left(x_{0}, y\right) D \varphi\left(x_{0}\right) & T_{x_{0}}^{\Delta}(y) \\
& +u\left(x_{0}, y\right) \frac{\mathcal{R}_{x_{0}}(y)}{\left|y-x_{0}\right|}+u_{x_{0}}^{\Delta}(y) D \varphi\left(x_{0}\right) T_{x_{0}}(y) .
\end{aligned}
$$

Since $\left|u\left(x_{0}, y\right)\right| \leq C$ by (A2), $\left|T_{x_{0}}(y)\right|=1$ by definition, and $\left|D \varphi\left(x_{0}\right)\right| \leq C_{\varphi}$ by the regularity assumption on $\varphi$, and $\left|T_{x_{0}}^{\Delta}(y)\right| \leq C\left|y-x_{0}\right|$ by (101), $\left|\mathcal{R}_{x_{0}}(y)\right| /\left|y-x_{0}\right| \leq$ $C_{\varphi}\left|y-x_{0}\right|$ by (99), and $\left|u_{x_{0}}^{\Delta}(y)\right|=\left|u_{x_{0}}^{\Delta}(y)-u_{x_{0}}^{\Delta}\left(x_{0}\right)\right| \leq C\left|y-x_{0}\right|$ by (A3)(iii), we obtain the bound

$$
\left|U_{x_{0}, \varphi}(y)\right| \leq C_{\varphi}\left|y-x_{0}\right|, \quad \forall y \in \Gamma_{x_{0}, d}, \quad y \neq x_{0} .
$$

In the above, the constant $C_{\varphi}$ depends on $\varphi$, but is independent of $x_{0}$ and $y$. Notice that the bound also holds when $y=x_{0}$ by definition of $U_{x_{0}, \varphi}$. To establish the Lipschitz property of this function, for any given $y \in \Gamma_{x_{0}, d}$ with $y \neq x_{0}$, we again consider an arbitrary curve $\gamma(\tau) \in \Gamma_{x_{0}, d}$ such that $\gamma(0)=y$. Then, by direct calculation 
using (103), (101), (99), (A2) and (A3)(iii), we find $\left|\frac{d}{d \tau} U_{x_{0}, \varphi}(\gamma(\tau))\right|_{\tau=0}\left|\leq C_{\varphi}\right| \gamma^{\prime}(0) \mid$. From this, we deduce

$$
\begin{gathered}
\left|D U_{x_{0}, \varphi}(y)\right| \leq C_{\varphi}, \quad \forall y \in \Gamma_{x_{0}, d}, \quad y \neq x_{0}, \\
U_{x_{0}, \varphi} \in C^{0,1}\left(\Gamma_{x_{0}, d}, \mathbb{R}^{k}\right),
\end{gathered}
$$

where, as before, the last result above follows from the fact that $U_{x_{0}, \varphi}(y)$ has a uniformly bounded surface derivative for almost every $y \in \Gamma_{x_{0}, d}$. Notice that the Lipschitz constant for $U_{x_{0}, \varphi}$ depends on $\varphi$, but is uniform in $x_{0} \in \Gamma$. Thus the result in part (i) is established.

For parts (ii) (a,b), let $x_{0} \in \Gamma$ be arbitrary and consider a local Cartesian coordinate map $y=\psi_{x_{0}}(\xi) \in \Gamma_{x_{0}, d}$ with $\xi \in \Omega_{x_{0}, d} \subset \mathbb{R}^{2}$, and a local polar coordinate map $y=\psi_{x_{0}}(\varpi(\rho, \widehat{\xi}))=\psi_{x_{0}}^{\text {polar }}(\rho, \widehat{\xi})$ with $(\rho, \widehat{\xi}) \in \Omega_{x_{0}, d}^{\text {polar }} \subset \mathbb{R}_{+} \times S$ and $\xi=\varpi(\rho, \widehat{\xi})=\rho \widehat{\xi}$. Introducing the Jacobian factor

$$
J(\xi)=\left(\operatorname{det}\left[\left(\frac{\partial \psi_{x_{0}}}{\partial \xi}\right)^{T} \frac{\partial \psi_{x_{0}}}{\partial \xi}\right]\right)^{1 / 2}
$$

we have the relations

$$
d A_{y}=J(\xi) d A_{\xi}=J(\rho \widehat{\xi}) \rho d \rho d S \widehat{\xi},
$$

where $d A_{y}$ is an area element in $\Gamma_{x_{0}, d}, d A_{\xi}$ is an area element in $\Omega_{x_{0}, d}$ and $d S \widehat{\xi}$ is an arclength element on $S$. By conditions (L2) and (L3) on $\Gamma$, we note that the Lyapunov radius $d>0$ can be chosen small enough such that

$$
1 \leq J(\xi) \leq 2, \quad \forall \xi \in \Omega_{x_{0}, d}, \quad x_{0} \in \Gamma .
$$

Moreover, if we let $L \geq 1$ denote the Lipschitz constant for $\psi_{x_{0}}$, which is uniform in $x_{0}$, then a straightforward argument using the definitions of $\Gamma_{x_{0}, \delta}$ and $\Omega_{x_{0}, \delta}$ shows that

$$
D_{x_{0}, \delta / L} \subset \Omega_{x_{0}, \delta} \subset D_{x_{0}, \delta}, \quad \forall \delta \in(0, d], \quad x_{0} \in \Gamma,
$$

where $D_{x_{0}, \delta}$ is the closed disc of radius $\delta>0$ at the origin.

Proceeding with (ii)(a,b), we consider the function $I: S \rightarrow \mathbb{R}$ defined by

$$
I(\widehat{\xi})=\max \left\{\int_{0}^{d / 2 L} J(\rho \widehat{\xi}) \rho d \rho, \int_{0}^{d / 2 L} J(-\rho \widehat{\xi}) \rho d \rho\right\} .
$$

Notice that $I(\widehat{\xi})$ is well-defined since $D_{x_{0}, d / 2 L} \subset \Omega_{x_{0}, d}$ by (109). From (110), we deduce $I(-\widehat{\xi})=I(\widehat{\xi})$, and from (110) and (108) we deduce, using a change of variable and the fact that $J(\xi) / J\left(\xi^{\prime}\right) \leq 2$ for all $\xi, \xi^{\prime} \in \Omega_{x_{0}, d}$,

$$
\frac{d^{2}}{8 L^{2}} \leq \int_{0}^{d / 2 L} J(\rho \widehat{\xi}) \rho d \rho \leq I(\widehat{\xi}) \leq \int_{0}^{d / L} J(\rho \widehat{\xi}) \rho d \rho \leq \frac{d^{2}}{L^{2}}
$$

where the second integral is also well-defined since $D_{x_{0}, d / L} \subset \Omega_{x_{0}, d}$ by (109). For any given choice of orthonormal basis in $T_{x_{0}} \Gamma$, we consider the components $\xi=$ $\left(\xi_{1}, \xi_{2}\right)$ and $\widehat{\xi}=\left(\widehat{\xi}_{1}, \widehat{\xi}_{2}\right)$, and the decomposition $S=S_{+} \cup S_{-} \subset \mathbb{R}^{2}$, where $S_{+}$and $S_{-}$are disjoint subsets defined as

$$
\begin{gathered}
S_{+}=\left\{\widehat{\xi} \in S \mid \widehat{\xi}_{2}>0 \text { or } \widehat{\xi}=(1,0)\right\}, \\
S_{-}=\left\{\widehat{\xi} \in S \mid \widehat{\xi}_{2}<0 \text { or } \widehat{\xi}=(-1,0)\right\} .
\end{gathered}
$$


Moreover, we consider functions $R_{+}: S_{+} \rightarrow \mathbb{R}$ and $R_{-}: S_{-} \rightarrow \mathbb{R}$ defined implicitly by

$$
\begin{aligned}
& \int_{0}^{R_{+}(\widehat{\xi})} J(\rho \widehat{\xi}) \rho d \rho=I(\widehat{\xi}), \quad \forall \widehat{\xi} \in S_{+}, \\
& \int_{0}^{R_{-}(\widehat{\xi})} J(\rho \widehat{\xi}) \rho d \rho=I(\widehat{\xi}), \quad \forall \widehat{\xi} \in S_{-},
\end{aligned}
$$

and from (111) and (110) we note that $R_{+}, R_{-} \in[d / 2 L, d / L]$. Furthermore, we use these functions to define subsets of $\Omega_{x_{0}, d}$ by

$$
\begin{aligned}
& \Omega_{x_{0},+}^{*}=\left\{\xi=\rho \widehat{\xi} \in \Omega_{x_{0}, d} \mid 0 \leq \rho \leq R_{+}(\widehat{\xi}), \widehat{\xi} \in S_{+}\right\}, \\
& \Omega_{x_{0},-}^{*}=\left\{\xi=\rho \widehat{\xi} \in \Omega_{x_{0}, d} \mid 0 \leq \rho \leq R_{-}(\widehat{\xi}), \widehat{\xi} \in S_{-}\right\},
\end{aligned}
$$

and we note that, since $R_{+}, R_{-} \in[d / 2 L, d / L]$ and $S_{+} \cup S_{-}=S$, we have

$$
D_{x_{0}, d / 2 L} \subset\left(\Omega_{x_{0},+}^{*} \cup \Omega_{x_{0},-}^{*}\right) \subset D_{x_{0}, d / L}, \quad \forall x_{0} \in \Gamma .
$$

Finally, we define a subset of $\Gamma_{x_{0}, d}$ by

$$
\Gamma_{x_{0}}^{*}=\left\{y=\psi_{x_{0}}(\xi) \in \Gamma_{x_{0}, d} \mid \xi \in \Omega_{x_{0},+}^{*} \cup \Omega_{x_{0},-}^{*}\right\} .
$$

The subset $\Gamma_{x_{0}}^{*}$ has the properties stated in part (ii)(a,b). Specifically, by (116), (115) and (109), it satisfies the uniformity condition

$$
\Gamma_{x_{0}, d / 2 L} \subset \Gamma_{x_{0}}^{*} \subset \Gamma_{x_{0}, d}, \quad \forall x_{0} \in \Gamma .
$$

Moreover, for any $\varphi \in C^{1,1}\left(\Gamma, \mathbb{R}^{k}\right)$, we have

$$
\begin{aligned}
\int_{\Gamma_{x_{0}}^{*}} & u_{x_{0}}^{\text {polar }}(0, \widehat{\xi}(y)) D \varphi\left(x_{0}\right) T_{x_{0}}(y) d A_{y} \\
= & \int_{S_{+}} \int_{0}^{R+(\widehat{\xi})} u_{x_{0}}^{\text {polar }}(0, \widehat{\xi}) D \varphi\left(x_{0}\right) T_{x_{0}}(\widehat{\xi}) J(\rho \widehat{\xi}) \rho d \rho d S_{\widehat{\xi}} \\
& +\int_{S_{-}} \int_{0}^{R_{-}(\widehat{\xi})} u_{x_{0}}^{\text {polar }}(0, \widehat{\xi}) D \varphi\left(x_{0}\right) T_{x_{0}}(\widehat{\xi}) J(\rho \widehat{\xi}) \rho d \rho d S \widehat{\xi},
\end{aligned}
$$

where we note that the unit tangent vector $T_{x_{0}}(y)$ is uniquely determined by the angular coordinate $\widehat{\xi}(y) \in S$, and hence we write $T_{x_{0}}(\widehat{\xi})$ in the last two integrals. Specifically, for any given orthonormal basis $\left\{t_{x_{0}, 1}, t_{x_{0}, 2}\right\}$ in $T_{x_{0}} \Gamma$, we have

$$
T_{x_{0}}(\widehat{\xi})=\widehat{\xi}_{1} t_{x_{0}, 1}+\widehat{\xi}_{2} t_{x_{0}, 2}
$$

Combining (118) with (113), we get

$$
\begin{aligned}
& \int_{\Gamma_{x_{0}}^{*}} u_{x_{0}}^{\text {polar }}(0, \widehat{\xi}(y)) D \varphi\left(x_{0}\right) T_{x_{0}}(y) d A_{y} \\
&=\int_{S_{+}} u_{x_{0}}^{\text {polar }}(0, \widehat{\xi}) D \varphi\left(x_{0}\right) T_{x_{0}}(\widehat{\xi}) I(\widehat{\xi}) d S_{\widehat{\xi}} \\
& \quad+\int_{S_{-}} u_{x_{0}}^{\text {polar }}(0, \widehat{\xi}) D \varphi\left(x_{0}\right) T_{x_{0}}(\widehat{\xi}) I(\widehat{\xi}) d S_{\widehat{\xi}}
\end{aligned}
$$

Since $u_{x_{0}}^{\text {polar }}(0, \widehat{\xi})$ is an even function on $S$ by $(\mathrm{A} 3)(\mathrm{i}, \mathrm{ii}), I(\widehat{\xi})$ is an even function on $S$ by (110), and $T_{x_{0}}(\widehat{\xi})$ is an odd function on $S$ by (119), and since the antipodal map $\hat{\xi} \mapsto-\widehat{\xi}$ is a length- and orientation-preserving map of $S$ onto $S$ which maps 
$S_{+}$onto $S_{-}$, we find that the two integrals on the right-hand side of (120) cancel, which implies

$$
\int_{\Gamma_{x_{0}}^{*}} u_{x_{0}}^{\text {polar }}(0, \widehat{\xi}(y)) D \varphi\left(x_{0}\right) T_{x_{0}}(y) d A_{y}=0, \quad \forall x_{0} \in \Gamma, \quad \varphi \in C^{1,1}\left(\Gamma, \mathbb{R}^{k}\right) .
$$

Thus the results in parts (ii) (a,b) are established.

For part (ii)(c), we use notation similar to that used in the proof of Lemma 4.3 and let $\beta \geq 10 L+1$ and $E_{\beta}^{*} \geq E_{0}$ be given numbers, where $E_{\beta}^{*}$ is sufficiently large such that $0<\beta h \leq d / 4 L$ for all $E \geq E_{\beta}^{*}$. Moreover, for any given $x_{0} \in \Gamma$, we consider the collections of surface elements $\Gamma^{e}, e=1, \ldots, E$, defined by

$$
I_{x_{0}}^{*}=\left\{e \mid \Gamma^{e} \subset \Gamma_{x_{0}}^{*}\right\} \quad \text { and } I_{x_{0}, \beta h}=\left\{e \mid \Gamma^{e} \subset \Gamma_{x_{0}, \beta h}\right\},
$$

and note that, by design, the sets $I_{x_{0}, \beta h} \subset I_{x_{0}}^{*}$ and $I_{x_{0}}^{*} \backslash I_{x_{0}, \beta h}$ are non-empty for all $E \geq E_{\beta}^{*}$. Moreover, for any given $\varphi \in C^{1,1}\left(\Gamma, \mathbb{R}^{k}\right)$, we consider the decomposition

$$
\sum_{e \in I_{x_{0}}^{*}} \sum_{1 \leq q \leq Q}^{\prime} F_{x_{0}}\left(\widehat{\xi}\left(x_{q}^{e}\right)\right) W_{q}^{e}=\mathcal{B}_{1}\left(x_{0}\right)+\mathcal{B}_{2}\left(x_{0}\right),
$$

where $F_{x_{0}}(\widehat{\xi})=u_{x_{0}}^{\text {polar }}(0, \widehat{\xi}) D \varphi\left(x_{0}\right) T_{x_{0}}(\widehat{\xi})$ and

$$
\begin{aligned}
& \mathcal{B}_{1}\left(x_{0}\right)=\sum_{e \in I_{x_{0}, \beta h}} \sum_{1 \leq q \leq Q}^{\prime} F_{x_{0}}\left(\widehat{\xi}\left(x_{q}^{e}\right)\right) W_{q}^{e}, \\
& \mathcal{B}_{2}\left(x_{0}\right)=\sum_{e \in I_{x_{0}}^{*} \backslash I_{x_{0}, \beta h}} \sum_{q=1}^{Q} F_{x_{0}}\left(\widehat{\xi}\left(x_{q}^{e}\right)\right) W_{q}^{e} .
\end{aligned}
$$

To establish the result, we show that the above sums satisfy the bounds $\left|\mathcal{B}_{1}\right| \leq C_{\varphi} h$ and $\left|\mathcal{B}_{2}\right| \leq C_{\varphi} h$ for all $E \geq E_{\beta}^{*}$ and $x_{0} \in \Gamma$, where the constant $C_{\varphi}$ depends on $\varphi$, but is independent of $x_{0}$. Results for the finite interval $E \in\left[E_{0}, E_{\beta}^{*}\right]$ are straightforward consequences of the boundedness of $F_{x_{0}}$ and will be omitted for brevity.

For the term $\mathcal{B}_{1}$ in (123), we use the fact that $\left|F_{x_{0}}\right| \leq C_{\varphi}$ by (A2) and (A3), $\left|I_{x_{0}, \beta h}\right| \leq C$ by (40), and $\sum_{q=1}^{Q} W_{q}^{e} \leq C h^{2}$ by (A5), to deduce

$$
\begin{aligned}
\left|\mathcal{B}_{1}\left(x_{0}\right)\right| & \leq \sum_{e \in I_{x_{0}, \beta h}} \sum_{1 \leq q \leq Q}^{\prime}\left|F_{x_{0}}\left(\widehat{\xi}\left(x_{q}^{e}\right)\right)\right| W_{q}^{e} \\
& \leq C_{\varphi} h^{2} \leq C_{\varphi} h, \quad \forall E \geq E_{\beta}^{*}, \quad x_{0} \in \Gamma,
\end{aligned}
$$

which establishes the result for $\mathcal{B}_{1}$.

For the second term $\mathcal{B}_{2}$ in (123) we notice that, for all $e \in I_{x_{0}}^{*} \backslash I_{x_{0}, \beta h}$, we must have $\Gamma^{e} \cap \Gamma_{x_{0},(\beta-1) h}=\emptyset$, or equivalently $\Omega^{e} \cap \Omega_{x_{0},(\beta-1) h}=\emptyset$ in terms of local Cartesian coordinate domains. Since $D_{x_{0},(\beta-1) h / L} \subset \Omega_{x_{0},(\beta-1) h}$ by (109), and $\beta \geq 10 L+1$ by assumption, this implies $0<10 h<|\xi|$ for all $\xi \in \Omega^{e}, e \in I_{x_{0}}^{*} \backslash I_{x_{0}, \beta h}$ and $E \geq E_{\beta}^{*}$. Moreover, for any two points $\xi, \xi^{\prime} \in \Omega^{e}$, with polar coordinates $\xi=\rho \widehat{\xi}$ and $\xi^{\prime}=\rho^{\prime} \widehat{\xi}^{\prime}$, we have

$$
\begin{aligned}
\left|\widehat{\xi}^{\prime}-\widehat{\xi}\right| & =\frac{\left|\left(\rho-\rho^{\prime}\right) \xi^{\prime}+\rho^{\prime}\left(\xi^{\prime}-\xi\right)\right|}{\rho^{\prime} \rho} \\
& \leq \frac{C h}{\rho}, \quad \forall \xi, \xi^{\prime} \in \Omega^{e}, \quad e \in I_{x_{0}}^{*} \backslash I_{x_{0}, \beta h}, \quad E \geq E_{\beta}^{*},
\end{aligned}
$$

where the inequality follows from the fact that $\operatorname{diam}\left(\Omega^{e}\right) \leq C \operatorname{diam}\left(\Gamma^{e}\right) \leq C h$ by the Lipschitz property of the coordinate maps $\psi_{x_{0}}$ and $\psi_{x_{0}}^{-1}$. Furthermore, since $u_{x_{0}}^{\text {polar }}(\rho, \widehat{\xi})$ is class $C^{0,1}$ in $(\rho, \widehat{\xi})$ by $(\mathrm{A} 3)(\mathrm{i})$, and $T_{x_{0}}(\widehat{\xi})$ is class $C^{\infty}$ in $\widehat{\xi}$ by (119), we notice that $F_{x_{0}}(\widehat{\xi})=u_{x_{0}}^{\text {polar }}(0, \widehat{\xi}) D \varphi\left(x_{0}\right) T_{x_{0}}(\widehat{\xi})$ is class $C^{0,1}$ in $\widehat{\xi}$ uniformly in $x_{0}$. 
Hence, for each $e \in I_{x_{0}}^{*} \backslash I_{x_{0}, \beta h}$ we have, using the fact that $W_{q}^{e}>0$, and considering each scalar component of $F_{x_{0}} \in \mathbb{R}^{k}$ separately,

$$
\min _{\xi \in \Omega^{e}} F_{x_{0}}(\widehat{\xi}) \leq \frac{\sum_{q=1}^{Q} F_{x_{0}}\left(\widehat{\xi}\left(x_{q}^{e}\right)\right) W_{q}^{e}}{\sum_{q=1}^{Q} W_{q}^{e}} \leq \max _{\xi \in \Omega^{e}} F_{x_{0}}(\widehat{\xi}) .
$$

From this we conclude, by the Intermediate Value Theorem, that for each scalar component of $F_{x_{0}}$ there exists an $\xi^{e}=\rho^{e} \widehat{\xi}^{e} \in \Omega^{e}$ with the property that

$$
\sum_{q=1}^{Q} F_{x_{0}}\left(\widehat{\xi}\left(x_{q}^{e}\right)\right) W_{q}^{e}=F_{x_{0}}\left(\widehat{\xi}^{e}\right) \sum_{q=1}^{Q} W_{q}^{e}, \quad \forall e \in I_{x_{0}}^{*} \backslash I_{x_{0}, \beta h}, \quad E \geq E_{\beta}^{*} .
$$

For convenience, we use the same symbol $\widehat{\xi}^{e}$ to denote the distinguished point for each component of $F_{x_{0}}$. In what follows, we will have need to consider the difference, denoted by $\Gamma_{x_{0}}^{*, \Delta}$, between the set $\Gamma_{x_{0}}^{*}$ and the subset $\bigcup_{e \in I_{x_{0}}^{*} \backslash I_{x_{0}}, \beta h} \Gamma^{e}$. From the definition of $I_{x_{0}}^{*}$ and $I_{x_{0}, \beta h}$, and the fact that $\operatorname{diam}\left(\Gamma^{e}\right) \leq h$, we deduce that

$$
\Gamma_{x_{0}}^{*, \Delta}=\Gamma_{x_{0}}^{*} \backslash\left(\bigcup_{e \in I_{x_{0}}^{*} \backslash I_{x_{0}, \beta h}} \Gamma^{e}\right) \subset \Gamma_{x_{0}, \beta h} \cup N\left(\partial \Gamma_{x_{0}}^{*}, h\right), \quad E \geq E_{\beta}^{*},
$$

where $N\left(\partial \Gamma_{x_{0}}^{*}, h\right)$ denotes the set of all $y \in \Gamma_{x_{0}}^{*} \operatorname{such}$ that $\operatorname{dist}\left(y, \partial \Gamma_{x_{0}}^{*}\right) \leq h$, that is, $N\left(\partial \Gamma_{x_{0}}^{*}, h\right)$ is the closed neighborhood of $\partial \Gamma_{x_{0}}^{*}$ in $\Gamma_{x_{0}}^{*}$ of size $h$. We note that $\Gamma_{x_{0}, \beta h}$ and $N\left(\partial \Gamma_{x_{0}}^{*}, h\right)$ are disjoint for $E_{\beta}^{*}$ sufficiently large. Moreover, from (113), (114) and (116), we deduce that the set $\partial \Gamma_{x_{0}}^{*}$ is a curve of class $C^{0,1}$.

Proceeding with the term $\mathcal{B}_{2}$ in (123), we have, using (129) and (125), and considering each scalar component separately,

$$
\begin{aligned}
\mathcal{B}_{2}\left(x_{0}\right) & =\sum_{e \in I_{x_{0}}^{*} \backslash I_{x_{0}, \beta h}} F_{x_{0}}\left(\widehat{\xi}^{e}\right) \sum_{q=1}^{Q} W_{q}^{e} \\
& =\sum_{e \in I_{x_{0}}^{*} \backslash I_{x_{0}, \beta h}} F_{x_{0}}\left(\widehat{\xi}^{e}\right)\left|\Gamma^{e}\right|+\sum_{e \in I_{x_{0}}^{*} \backslash I_{x_{0}}, \beta h} F_{x_{0}}\left(\widehat{\xi}^{e}\right)\left(\sum_{q=1}^{Q} W_{q}^{e}-\left|\Gamma^{e}\right|\right) .
\end{aligned}
$$

Using the fact that $\int_{\Gamma_{x_{0}}^{*}} F_{x_{0}}(\widehat{\xi}(y)) d A_{y}=0$ by (121), and the definition of $\Gamma_{x_{0}}^{*, \Delta}$ in (130), we get

$$
\begin{aligned}
\mathcal{B}_{2}\left(x_{0}\right)= & \sum_{e \in I_{x_{0}}^{*} \backslash I_{x_{0}, \beta h}} F_{x_{0}}\left(\widehat{\xi}^{e}\right)\left|\Gamma^{e}\right|+\sum_{e \in I_{x_{0}}^{*} \backslash I_{x_{0}, \beta h}} F_{x_{0}}\left(\widehat{\xi}^{e}\right)\left(\sum_{q=1}^{Q} W_{q}^{e}-\left|\Gamma^{e}\right|\right) \\
= & -\sum_{e \in I_{x_{0}}^{*} \backslash I_{x_{0}}, \beta h} \int_{\Gamma^{e}} F_{x_{0}}(\widehat{\xi}(y)) d A_{y} \\
& \left.+\sum_{e \in I_{x_{0}}^{*} \backslash I_{x_{0}, \beta h}} F_{x_{0}}\left(\widehat{\xi}^{e}\right)\left(\sum_{q=1}^{Q} W_{q}^{e}-\left|\Gamma^{e}\right|\right)-\int_{\Gamma_{x_{0}}^{*, \Delta}} F_{x_{0}}(\widehat{\xi}(y)) d A_{y}\right) \\
= & \mathcal{B}_{21}\left(x_{0}\right)+A_{y} \\
& +\mathcal{B}_{22}\left(x_{0}\right)-\mathcal{B}_{23}\left(x_{0}\right),
\end{aligned}
$$


where $\mathcal{B}_{21}\left(x_{0}\right), \mathcal{B}_{22}\left(x_{0}\right)$ and $\mathcal{B}_{23}\left(x_{0}\right)$ denote the three terms in the middle equation of (132).

For the term $\mathcal{B}_{21}$ in (132), we notice that, by the Lipschitz property of $F_{x_{0}}(\widehat{\xi})$ and (127), we have

$$
\begin{aligned}
\left|F_{x_{0}}\left(\widehat{\xi}^{e}\right)-F_{x_{0}}(\widehat{\xi}(y))\right| & \leq C_{\varphi}\left|\widehat{\xi}^{e}-\widehat{\xi}(y)\right| \\
& \leq \frac{C_{\varphi} h}{\rho(y)} \leq \frac{C_{\varphi} h}{\left|y-x_{0}\right|}, \quad \forall e \in I_{x_{0}}^{*} \backslash I_{x_{0}, \beta h}, \quad E \geq E_{\beta}^{*},
\end{aligned}
$$

where the last inequality follows from the Lipschitz property of the coordinate map $\psi_{x_{0}}$ and the fact that $\left|y-x_{0}\right|=\left|\psi_{x_{0}}(\xi(y))-\psi_{x_{0}}(0)\right| \leq C|\xi(y)|$ and $\rho(y)=|\xi(y)|$. Hence, for the term $\mathcal{B}_{21}$ we obtain the uniform bound

$$
\begin{aligned}
\left|\mathcal{B}_{21}\left(x_{0}\right)\right| & \leq \sum_{e \in I_{x_{0}}^{*} \backslash I_{x_{0}, \beta h}} \int_{\Gamma^{e}}\left|F_{x_{0}}\left(\widehat{\xi}^{e}\right)-F_{x_{0}}(\widehat{\xi}(y))\right| d A_{y} \\
& \leq C_{\varphi} h \int_{\Gamma_{x_{0}}^{*}} \frac{1}{\left|y-x_{0}\right|} d A_{y} \leq C_{\varphi} h, \quad \forall E \geq E_{\beta}^{*}, \quad x_{0} \in \Gamma,
\end{aligned}
$$

where the last inequality follows from a direct estimate of the integral using polar coordinates, which shows that the integral is bounded, and the constant $C_{\varphi}$ depends on $\varphi$, but is independent of $x_{0}$. Thus the result for $\mathcal{B}_{21}$ is established.

For the term $\mathcal{B}_{22}$ in (132), we first use the quadrature error bound in (A6) for a rule of order $\ell \geq 1$ applied to a constant function to obtain

$$
\left|\sum_{q=1}^{Q} W_{q}^{e}-\right| \Gamma^{e}||=\left|\sum_{q=1}^{Q} W_{q}^{e}-\int_{\Gamma^{e}} d A_{y}\right| \leq C\left|\Gamma^{e}\right| h, \forall e \in I_{x_{0}}^{*} \backslash I_{x_{0}, \beta h}, E \geq E_{\beta}^{*}
$$

Using the above, together with the fact that $\left|F_{x_{0}}(\widehat{\xi})\right| \leq C_{\varphi}$ by (A2), (A3) and the definition of $F_{x_{0}}$, we then obtain the uniform bound

$$
\begin{aligned}
\left|\mathcal{B}_{22}\left(x_{0}\right)\right| & \leq \sum_{e \in I_{x_{0}}^{*} \backslash I_{x_{0}, \beta h}}\left|F_{x_{0}}\left(\widehat{\xi}^{e}\right)\right|\left|\sum_{q=1}^{Q} W_{q}^{e}-\right| \Gamma^{e}|| \\
& \leq \sum_{e \in I_{x_{0}}^{*} \backslash I_{x_{0}, \beta h}} C_{\varphi}\left|\Gamma^{e}\right| h \leq C_{\varphi}\left|\Gamma_{x_{0}}^{*}\right| h \leq C_{\varphi} h, \quad \forall E \geq E_{\beta}^{*}, \quad x_{0} \in \Gamma,
\end{aligned}
$$

where the constant $C_{\varphi}$ depends on $\varphi$, but is independent of $x_{0}$. Thus the result for $\mathcal{B}_{22}$ is established.

For the term $\mathcal{B}_{23}$ in (132), we again use the fact that $\left|F_{x_{0}}(\widehat{\xi})\right| \leq C_{\varphi}$ as above, and use the inclusion result for $\Gamma_{x_{0}}^{*, \Delta}$ in (130), to get

$$
\begin{aligned}
\left|\mathcal{B}_{23}\left(x_{0}\right)\right| & \leq \int_{\Gamma_{x_{0}}^{*, \Delta}}\left|F_{x_{0}}(\widehat{\xi}(y))\right| d A_{y} \\
& \leq C_{\varphi} \int_{\Gamma_{x_{0}, \beta h}} d A_{y}+C_{\varphi} \int_{N\left(\partial \Gamma_{x_{0}}^{*}, h\right)} d A_{y} \leq C_{\varphi} h, \quad \forall E \geq E_{\beta}^{*}, \quad x_{0} \in \Gamma,
\end{aligned}
$$

where the last inequality follows from the definition of the patch $\Gamma_{x_{0}, \beta h}$ and the set $N\left(\partial \Gamma_{x_{0}}^{*}, h\right)$, and the constant $C_{\varphi}$ depends on $\varphi$, but is independent of $x_{0}$. Thus the result for $\mathcal{B}_{23}$ is established. The result for $\mathcal{B}_{2}$ follows from (137), (136) and (134), and the result stated in part (ii)(c) follows from these, together with (132), (126) and (123). 
4.3. Proof of main result. Here we combine the collective compactness result in Theorem 4.1 and the results in Lemmas 4.2 4.6 to establish our main result in Theorem 3.1. We consider the locally-corrected Nyström method defined in (15)(23) with a quadrature rule of arbitrary order $\ell \geq 1$, a local polynomial correction of arbitrary degree $p \geq 0$, and a surface with regularity index $m \geq 0$.

For part (i), consider any $\ell \geq 1, p \geq 0$ and $m \geq 0$. Then, by Lemma 4.5, the operators $\mathcal{A}$ and $\mathcal{A}_{n}\left(n \geq n_{0}\right)$ satisfy the collective compactness conditions (C1)(C3). Hence, by Theorem 4.1. there exist constants $C>0$ and $N \geq n_{0}$ such that $c \varphi_{n}-\mathcal{A}_{n} \varphi_{n}=f$ is uniquely solvable for $\varphi_{n}$, and moreover,

$$
\left\|\varphi_{n}-\varphi\right\| \leq C\left\|\mathcal{A}_{n} \varphi-\mathcal{A} \varphi\right\|, \quad \forall n \geq N .
$$

By $(\mathrm{C} 1)$ and the fact that $\varphi \in C^{0}\left(\Gamma, \mathbb{R}^{k}\right)$, we have $\left(\mathcal{A}_{n} \varphi\right)(x) \rightarrow(\mathcal{A} \varphi)(x)$ uniformly in $x \in \Gamma$, which in view of (138) implies

$$
\left\|\varphi_{n}-\varphi\right\| \rightarrow 0 \quad \text { as } n \rightarrow \infty, \quad \forall \ell \geq 1, p \geq 0, m \geq 0 .
$$

Thus the result in part (i) of the main theorem is established.

For convenience, we next consider part (iii), and will consider part (ii) afterwards since it requires a special treatment. Accordingly, consider any $\ell \geq 1, p \geq 1$ and $m \geq 1$, and assume $\varphi \in C^{m, 1}\left(\Gamma, \mathbb{R}^{k}\right)$. In view of (138), we seek a bound for $\left\|\mathcal{A}_{n} \varphi-\mathcal{A} \varphi\right\|$. By definition of $\mathcal{A}_{n}$ and $\mathcal{A}$, we have

$$
\left\|\mathcal{A}_{n} \varphi-\mathcal{A} \varphi\right\| \leq\left\|\mathcal{G}_{n} \varphi-\mathcal{G} \varphi\right\|+\left\|\mathcal{H}_{n} \varphi-\mathcal{H} \varphi\right\|,
$$

where $\mathcal{G}$ and $\mathcal{H}$ are the operators defined in (7), and $\mathcal{G}_{n}$ and $\mathcal{H}_{n}$ are the operators defined in (15); we continue to use subscripts $n \rightarrow \infty$ in place of $h_{n} \rightarrow 0$.

For the first term in (140), we let $x_{0} \in \Gamma$ be arbitrary and consider the function $\left(G_{x_{0}} \varphi\right)(y)=G\left(x_{0}, y\right) \varphi(y)$. Since $\varphi \in C^{m, 1}\left(\Gamma, \mathbb{R}^{k}\right)$ by assumption, and $G \in$ $C^{m, 1}\left(\Gamma \times \Gamma, \mathbb{R}^{k \times k}\right)$ by (A1), it follows that $G_{x_{0}} \varphi \in C^{m, 1}\left(\Gamma, \mathbb{R}^{k}\right)$, where the Lipschitz constant for each derivative is uniform in $x_{0}$. In view of the quadrature error bound in (A6) for a rule of order $\ell$, we get, by definition of $\mathcal{G}$ and $\mathcal{G}_{n}$,

$$
\begin{aligned}
& \left|\left(\mathcal{G}_{n} \varphi\right)\left(x_{0}\right)-(\mathcal{G} \varphi)\left(x_{0}\right)\right| \\
& =\left|\sum_{e=1}^{E} \sum_{q=1}^{Q} G\left(x_{0}, x_{q}^{e}\right) \varphi\left(x_{q}^{e}\right) W_{q}^{e}-\int_{\Gamma} G\left(x_{0}, y\right) \varphi(y) d A_{y}\right| \\
& \quad \leq \sum_{e=1}^{E}\left|\sum_{q=1}^{Q}\left(G_{x_{0}} \varphi\right)\left(x_{q}^{e}\right) W_{q}^{e}-\int_{\Gamma^{e}}\left(G_{x_{0}} \varphi\right)(y) d A_{y}\right| \\
& \leq C_{\varphi} h^{\min (\ell, m+1)}, \quad \forall n \geq N, \quad x_{0} \in \Gamma .
\end{aligned}
$$

In the above, the constant $C_{\varphi}$ depends on $\varphi$, but is independent of $x_{0}$. From this we deduce the bound

$$
\left\|\mathcal{G}_{n} \varphi-\mathcal{G} \varphi\right\| \leq C_{\varphi} h^{\min (\ell, m+1)}, \quad \forall n \geq N .
$$

To bound the second term in (140), we let $T_{x_{0}}^{j} \varphi: \Gamma_{x_{0}, d} \rightarrow \mathbb{R}^{k}$ be the local Taylor polynomial of degree $j$ for $\varphi$ at $x_{0} \in \Gamma$. Just as in (21), the local polynomial $\left(T_{x_{0}}^{j} \varphi\right)(z)$ is defined using local Cartesian coordinates $\xi_{x_{0}}(z)$ in $\Gamma_{x_{0}, d}$ as given in (11). Specifically, we have

$$
\left(T_{x_{0}}^{j} \varphi\right)(z)=\left.\sum_{\sigma=0}^{j} \frac{1}{\sigma !} \frac{d^{\sigma}}{d \tau^{\sigma}}\right|_{\tau=0}\left(\varphi \circ \psi_{x_{0}}\right)\left(\tau \xi_{x_{0}}(z)\right)=\sum_{|\alpha| \leq j} B_{x_{0}, \alpha} \xi_{x_{0}}^{\alpha}(z)
$$


where the coefficients $B_{x_{0}, \alpha}$ are identified with the partial derivatives of $\varphi \circ \psi_{x_{0}}$ with respect to $\xi_{x_{0}}$, with appropriate multiplicity due to equality of mixed partials, and including the factorial scaling, evaluated at the surface point $x_{0}$ or equivalently $\xi_{x_{0}}=0$ in local Cartesian coordinates. Since $\varphi \in C^{m, 1}\left(\Gamma, \mathbb{R}^{k}\right)$, it follows that $T_{x_{0}}^{j} \varphi$ is well-defined for any $0 \leq j \leq m$. Moreover, in view of the Lipschitz properties of the coordinates $\xi_{x_{0}}(z)$ for a surface $\Gamma$ of class $C^{m+1,1}$, it follows that $T_{x_{0}}^{j} \varphi \in$ $C^{m+1,1}\left(\Gamma_{x_{0}, d}, \mathbb{R}^{k}\right)$, where the Lipschitz constant for each derivative is uniform in $x_{0}$. Furthermore, by definition of the locally-corrected operator $\mathcal{H}_{n}$, the relation in (20) holds, which implies

$$
\left(\mathcal{H}_{n} \eta_{x_{0}} T_{x_{0}}^{j} \varphi\right)\left(x_{0}\right)=\left(\mathcal{H} \eta_{x_{0}} T_{x_{0}}^{j} \varphi\right)\left(x_{0}\right), \quad \forall 0 \leq j \leq p, \quad x_{0} \in \Gamma
$$

Here $\eta_{x_{0}} \in C^{m, 1}(\Gamma,[0,1])$ is any given cut-off function as described earlier. Notice that $\eta_{x_{0}} T_{x_{0}}^{j} \varphi$ is defined at all points of the surface and $\eta_{x_{0}} T_{x_{0}}^{j} \varphi \in C^{m, 1}\left(\Gamma, \mathbb{R}^{k}\right)$, where the Lipschitz constant for each derivative is uniform in $x_{0}$.

In what follows, we will have need to consider the function $\varphi_{x_{0}}^{\Delta} \in C^{m, 1}\left(\Gamma, \mathbb{R}^{k}\right)$ defined by

$$
\varphi_{x_{0}}^{\Delta}(y)=\left[\varphi-\eta_{x_{0}} T_{x_{0}}^{j} \varphi\right](y), \quad j=\min (p, m) .
$$

From the definition of the local Taylor polynomial $T_{x_{0}}^{j} \varphi$, and the fact that $\eta_{x_{0}}$ is identically equal to one in a fixed neighborhood of $x_{0}$, we deduce that

$$
\begin{gathered}
\left|\varphi_{x_{0}}^{\Delta}(y)\right| \leq C_{\varphi}\left|y-x_{0}\right|^{j+1}, \quad\left|D^{s} \varphi_{x_{0}}^{\Delta}(y)\right| \leq C_{\varphi}\left|y-x_{0}\right|^{j+1-s} \\
0 \leq s \leq j, \quad x_{0}, y \in \Gamma
\end{gathered}
$$

where $D^{s} \varphi_{x_{0}}^{\Delta}$ denotes the surface derivative of order $s$. For any given $x_{0}$, we will also have need to consider the function $\left(H_{x_{0}} \varphi_{x_{0}}^{\Delta}\right)(y)=H\left(x_{0}, y\right) \varphi_{x_{0}}^{\Delta}(y)$. Using (146), together with (A2) and (A3) with $\mu=1$, we find

$$
\begin{gathered}
\left|\left(H_{x_{0}} \varphi_{x_{0}}^{\Delta}\right)(y)\right| \leq C_{\varphi}\left|y-x_{0}\right|^{j}, \quad\left|D^{s}\left(H_{x_{0}} \varphi_{x_{0}}^{\Delta}\right)(y)\right| \leq C_{\varphi}\left|y-x_{0}\right|^{j-s} \\
0 \leq s \leq j, \quad x_{0}, y \in \Gamma .
\end{gathered}
$$

Here and above $C_{\varphi}$ is a constant depending on $\varphi$, but is independent of $x_{0}$ and $y$. From (147) with $s=j$, we find that the surface derivative of $H_{x_{0}} \varphi_{x_{0}}^{\Delta}$ of order $j$ is uniformly bounded, namely $\left|D^{j}\left(H_{x_{0}} \varphi_{x_{0}}^{\Delta}\right)(y)\right| \leq C_{\varphi}$ for all $x_{0}, y \in \Gamma$. Thus it follows that $H_{x_{0}} \varphi_{x_{0}}^{\Delta} \in C^{j-1,1}\left(\Gamma, \mathbb{R}^{k}\right)$, where $j=\min (p, m)$ and the Lipschitz constant for each derivative is uniform in $x_{0}$. This last regularity result provides a hint of the delicate nature of the case $p=0$ that will be considered later.

Proceeding with the second term in (140), we consider an arbitrary $x_{0} \in \Gamma$ and use (144) and (145) to write

$$
\left(\mathcal{H}_{n} \varphi-\mathcal{H} \varphi\right)\left(x_{0}\right)=\left(\mathcal{H}_{n} \varphi_{x_{0}}^{\Delta}-\mathcal{H} \varphi_{x_{0}}^{\Delta}\right)\left(x_{0}\right)
$$


Using the definitions of $\mathcal{H}$ and $\mathcal{H}_{n}$ in (148), and the fact that $\zeta_{b}+\widehat{\zeta}_{b}=1$, we get

$$
\begin{aligned}
& \left(\mathcal{H}_{n} \varphi-\mathcal{H} \varphi\right)\left(x_{0}\right) \\
& =\sum_{b=1}^{n} \zeta_{b}\left(x_{0}\right) H\left(x_{0}, x_{b}\right) \varphi_{x_{0}}^{\Delta}\left(x_{b}\right) W_{b}+\sum_{b=1}^{n} \widehat{\zeta}_{b}\left(x_{0}\right) R_{x_{0}}\left(x_{b}\right) \varphi_{x_{0}}^{\Delta}\left(x_{b}\right) \\
& \quad-\int_{\Gamma} H\left(x_{0}, y\right) \varphi_{x_{0}}^{\Delta}(y) d A_{y}, \\
& =\sum_{b=1}^{n} H\left(x_{0}, x_{b}\right) \varphi_{x_{0}}^{\Delta}\left(x_{b}\right) W_{b}-\int_{\Gamma} H\left(x_{0}, y\right) \varphi_{x_{0}}^{\Delta}(y) d A_{y} \\
& \quad+\sum_{b=1}^{n} \widehat{\zeta}_{b}\left(x_{0}\right) R_{x_{0}}\left(x_{b}\right) \varphi_{x_{0}}^{\Delta}\left(x_{b}\right)-\sum_{b=1}^{n} \widehat{\zeta}_{b}\left(x_{0}\right) H\left(x_{0}, x_{b}\right) \varphi_{x_{0}}^{\Delta}\left(x_{b}\right) W_{b} .
\end{aligned}
$$

For convenience, we decompose the above into three parts, namely

$$
\left(\mathcal{H}_{n} \varphi-\mathcal{H} \varphi\right)\left(x_{0}\right)=\mathcal{M}_{1}\left(x_{0}\right)+\mathcal{M}_{2}\left(x_{0}\right)-\mathcal{M}_{3}\left(x_{0}\right),
$$

where

$$
\begin{aligned}
& \mathcal{M}_{1}\left(x_{0}\right)=\sum_{b=1}^{n} H\left(x_{0}, x_{b}\right) \varphi_{x_{0}}^{\Delta}\left(x_{b}\right) W_{b}-\int_{\Gamma} H\left(x_{0}, y\right) \varphi_{x_{0}}^{\Delta}(y) d A_{y}, \\
& \mathcal{M}_{2}\left(x_{0}\right)=\sum_{b=1}^{n} \widehat{\zeta}_{b}\left(x_{0}\right) R_{x_{0}}\left(x_{b}\right) \varphi_{x_{0}}^{\Delta}\left(x_{b}\right), \\
& \mathcal{M}_{3}\left(x_{0}\right)=\sum_{b=1}^{n} \widehat{\zeta}_{b}\left(x_{0}\right) H\left(x_{0}, x_{b}\right) \varphi_{x_{0}}^{\Delta}\left(x_{b}\right) W_{b} .
\end{aligned}
$$

To establish the required result, we proceed to bound each of $\mathcal{M}_{1}, \mathcal{M}_{2}$ and $\mathcal{M}_{3}$ uniformly in $x_{0}$.

For the term $\mathcal{M}_{1}$ in (150), we note that $H_{x_{0}} \varphi_{x_{0}}^{\Delta} \in C^{j-1,1}\left(\Gamma, \mathbb{R}^{k}\right)$ by the discussion following (147). In view of the quadrature error bound in (A6) for a rule of order $\ell$, we get

$$
\begin{aligned}
\left|\mathcal{M}_{1}\left(x_{0}\right)\right| & =\left|\sum_{e=1}^{E} \sum_{q=1}^{Q}\left(H_{x_{0}} \varphi_{x_{0}}^{\Delta}\right)\left(x_{q}^{e}\right) W_{q}^{e}-\int_{\Gamma}\left(H_{x_{0}} \varphi_{x_{0}}^{\Delta}\right)(y) d A_{y}\right| \\
& \leq \sum_{e=1}^{E}\left|\sum_{q=1}^{Q}\left(H_{x_{0}} \varphi_{x_{0}}^{\Delta}\right)\left(x_{q}^{e}\right) W_{q}^{e}-\int_{\Gamma^{e}}\left(H_{x_{0}} \varphi_{x_{0}}^{\Delta}\right)(y) d A_{y}\right| \\
& \leq C_{\varphi} h^{\min (\ell, j)}=C_{\varphi} h^{\min (\ell, p, m)}, \quad \forall n \geq N, \quad x_{0} \in \Gamma,
\end{aligned}
$$

where the last equality follows from the fact that $j=\min (p, m)$. In the above, the constant $C_{\varphi}$ depends on $\varphi$, but is independent of $x_{0}$. Thus a uniform bound for $\mathcal{M}_{1}$ is established.

For the term $\mathcal{M}_{2}$ in (150), we consider the index set $J_{x_{0}}=\left\{b \mid \widehat{\zeta}_{b}\left(x_{0}\right)>0\right\}$, and we note by the same arguments that led to (84), that the number of elements in this set is uniformly bounded, namely $\left|J_{x_{0}}\right| \leq C$ for all $n \geq N$ and $x_{0} \in \Gamma$. From (152) we get, using the definition of $J_{x_{0}}$ and the fact that $0 \leq \widehat{\zeta}_{b} \leq 1$,

$$
\left|\mathcal{M}_{2}\left(x_{0}\right)\right| \leq \sum_{b=1}^{n}\left|\widehat{\zeta}_{b}\left(x_{0}\right) R_{x_{0}}\left(x_{b}\right) \varphi_{x_{0}}^{\Delta}\left(x_{b}\right)\right| \leq \sum_{b \in J_{x_{0}}}\left|R_{x_{0}}\left(x_{b}\right)\right|\left|\varphi_{x_{0}}^{\Delta}\left(x_{b}\right)\right| .
$$


Since $\left|R_{x_{0}}\left(x_{b}\right)\right| \leq C$ by Lemma 4.4(iii), $\left|\varphi_{x_{0}}^{\Delta}\left(x_{b}\right)\right| \leq C_{\varphi}\left|x_{b}-x_{0}\right|^{j+1}$ by (146), and $\left|x_{b}-x_{0}\right| \leq C h$ for all $b \in J_{x_{0}}$ by definition of $J_{x_{0}}$ and (A7), we get

$$
\left|\mathcal{M}_{2}\left(x_{0}\right)\right| \leq C_{\varphi} h^{j+1}=C_{\varphi} h^{\min (p+1, m+1)}, \quad \forall n \geq N, \quad x_{0} \in \Gamma,
$$

where the last equality follows from the fact that $j=\min (p, m)$. As before, the constant $C_{\varphi}$ depends on $\varphi$, but is independent of $x_{0}$. Thus a uniform bound for $\mathcal{M}_{2}$ is established.

For the term $\mathcal{M}_{3}$ in (150), we again use the definition of $J_{x_{0}}$ and the fact that $0 \leq \widehat{\zeta}_{b} \leq 1$, together with (153), to write

$$
\left|\mathcal{M}_{3}\left(x_{0}\right)\right| \leq \sum_{b=1}^{n}\left|\widehat{\zeta}_{b}\left(x_{0}\right) H\left(x_{0}, x_{b}\right) \varphi_{x_{0}}^{\Delta}\left(x_{b}\right) W_{b}\right| \leq \sum_{b \in J_{x_{0}}}\left|\left(H_{x_{0}} \varphi_{x_{0}}^{\Delta}\right)\left(x_{b}\right)\right| W_{b} .
$$

Since $\left|\left(H_{x_{0}} \varphi_{x_{0}}^{\Delta}\right)\left(x_{b}\right)\right| \leq C_{\varphi}\left|x_{b}-x_{0}\right|^{j}$ by (147), and $\left|x_{b}-x_{0}\right| \leq C h$ for all $b \in J_{x_{0}}$ by definition of $J_{x_{0}}$ and (A7), and $W_{b} \leq C h^{2}$ by (A5), we get

$$
\left|\mathcal{M}_{3}\left(x_{0}\right)\right| \leq C_{\varphi} h^{j+2}=C_{\varphi} h^{\min (p+2, m+2)}, \quad \forall n \geq N, \quad x_{0} \in \Gamma,
$$

where the last equality follows from the fact that $j=\min (p, m)$. Again, the constant $C_{\varphi}$ depends on $\varphi$, but is independent of $x_{0}$. Thus a uniform bound for $\mathcal{M}_{3}$ is established. Combining (158), (156), (154) and (150), we obtain a bound for the second term in (140), namely

$$
\left\|\mathcal{H}_{n} \varphi-\mathcal{H} \varphi\right\| \leq C_{\varphi} h^{\min (\ell, p, m)}, \quad \forall n \geq N .
$$

Combining (159), (142), (140) and (138), we get

$$
\left\|\varphi_{n}-\varphi\right\| \leq C_{\varphi} h^{\min (\ell, p, m)} \quad \text { as } \quad n \rightarrow \infty, \quad \forall \ell \geq 1, p \geq 1, m \geq 1 .
$$

Thus the result stated in part (iii) of the main theorem is established.

For part (ii) of the main theorem, we assume $p=0$ and consider any $\ell \geq 1$ and $m \geq 1$. In view of (138), we again seek a bound for $\left\|\mathcal{A}_{n} \varphi-\mathcal{A} \varphi\right\|$ and consider the decomposition in (140). The term $\left\|\mathcal{G}_{n} \varphi-\mathcal{G} \varphi\right\|$ can be bound exactly as in (142). Hence we focus on the term $\left\|\mathcal{H}_{n} \varphi-\mathcal{H} \varphi\right\|$, which requires a different treatment than before due to the limited regularity of $H_{x_{0}} \varphi_{x_{0}}^{\Delta}$ in the case $p=0$. To this end, we consider an arbitrary $x_{0} \in \Gamma$ and let $\Gamma_{x_{0}}^{*}$ and $I_{x_{0}}^{*}$ be as in Lemma 4.6(ii). Then, using (148) and the definitions of $\mathcal{H}$ and $\mathcal{H}_{n}$, we get

$$
\begin{aligned}
& \left(\mathcal{H}_{n} \varphi-\mathcal{H} \varphi\right)\left(x_{0}\right) \\
& =\sum_{b=1}^{n} \zeta_{b}\left(x_{0}\right) H\left(x_{0}, x_{b}\right) \varphi_{x_{0}}^{\Delta}\left(x_{b}\right) W_{b}+\sum_{b=1}^{n} \widehat{\zeta}_{b}\left(x_{0}\right) R_{x_{0}}\left(x_{b}\right) \varphi_{x_{0}}^{\Delta}\left(x_{b}\right) \\
& -\int_{\Gamma} H\left(x_{0}, y\right) \varphi_{x_{0}}^{\Delta}(y) d A_{y}, \\
& =\sum_{e \in I_{x_{0}}^{*}}\left[\sum_{q=1}^{Q} \zeta_{q}^{e}\left(x_{0}\right) H\left(x_{0}, x_{q}^{e}\right) \varphi_{x_{0}}^{\Delta}\left(x_{q}^{e}\right) W_{q}^{e}-\int_{\Gamma^{e}} H\left(x_{0}, y\right) \varphi_{x_{0}}^{\Delta}(y) d A_{y}\right] \\
& +\sum_{e \notin I_{x_{0}}^{*}}\left[\sum_{q=1}^{Q} \zeta_{q}^{e}\left(x_{0}\right) H\left(x_{0}, x_{q}^{e}\right) \varphi_{x_{0}}^{\Delta}\left(x_{q}^{e}\right) W_{q}^{e}-\int_{\Gamma^{e}} H\left(x_{0}, y\right) \varphi_{x_{0}}^{\Delta}(y) d A_{y}\right] \\
& +\sum_{b=1}^{n} \widehat{\zeta}_{b}\left(x_{0}\right) R_{x_{0}}\left(x_{b}\right) \varphi_{x_{0}}^{\Delta}\left(x_{b}\right) .
\end{aligned}
$$


For the first term in the first bracketed expression in (161), we observe that $\zeta_{q}^{e}\left(x_{0}\right) H\left(x_{0}, x_{q}^{e}\right) \varphi_{x_{0}}^{\Delta}\left(x_{q}^{e}\right)=0$ if $x_{q}^{e}=x_{0}$ by (147) and (A7). Thus, using the notation from Lemma 4.6 and the fact that $\zeta_{q}^{e}+\widehat{\zeta}_{q}^{e}=1$, we have

$$
\begin{aligned}
& \sum_{q=1}^{Q} \zeta_{q}^{e}\left(x_{0}\right) H\left(x_{0}, x_{q}^{e}\right) \varphi_{x_{0}}^{\Delta}\left(x_{q}^{e}\right) W_{q}^{e} \\
& =\sum_{1 \leq q \leq Q}^{\prime} \zeta_{q}^{e}\left(x_{0}\right) H\left(x_{0}, x_{q}^{e}\right) \varphi_{x_{0}}^{\Delta}\left(x_{q}^{e}\right) W_{q}^{e}, \\
& =\sum_{1 \leq q \leq Q}^{\prime} H\left(x_{0}, x_{q}^{e}\right) \varphi_{x_{0}}^{\Delta}\left(x_{q}^{e}\right) W_{q}^{e}-\sum_{1 \leq q \leq Q}^{\prime} \widehat{\zeta}_{q}^{e}\left(x_{0}\right) H\left(x_{0}, x_{q}^{e}\right) \varphi_{x_{0}}^{\Delta}\left(x_{q}^{e}\right) W_{q}^{e}
\end{aligned}
$$

Using the definition of $U_{x_{0}, \varphi}$ given in (96), and the definition of $\varphi_{x_{0}}^{\Delta}$ given in (145) with $p=0$, and setting $\eta_{x_{0}}$ to unity in the case $p=0$, we get

$$
\begin{aligned}
& \sum_{q=1}^{Q} \zeta_{q}^{e}\left(x_{0}\right) H\left(x_{0}, x_{q}^{e}\right) \varphi_{x_{0}}^{\Delta}\left(x_{q}^{e}\right) W_{q}^{e} \\
& =\sum_{1 \leq q \leq Q}^{\prime} U_{x_{0}, \varphi}\left(x_{q}^{e}\right) W_{q}^{e}-\sum_{1 \leq q \leq Q}^{\prime} \widehat{\zeta}_{q}^{e}\left(x_{0}\right) H\left(x_{0}, x_{q}^{e}\right) \varphi_{x_{0}}^{\Delta}\left(x_{q}^{e}\right) W_{q}^{e} \\
& \quad+\sum_{1 \leq q \leq Q}^{\prime} u_{x_{0}}^{\text {polar }}\left(0, \widehat{\xi}\left(x_{q}^{e}\right)\right) D \varphi\left(x_{0}\right) T_{x_{0}}\left(x_{q}^{e}\right) W_{q}^{e} .
\end{aligned}
$$

Similarly, for the second term in the first bracketed expression in (161), we use Lemma 4.6(ii)(b) and (130), together with the definitions of $U_{x_{0}, \varphi}$ and $\varphi_{x_{0}}^{\Delta}$, to write

$$
\begin{aligned}
& \sum_{e \in I_{x_{0}}^{*}} \int_{\Gamma^{e}} H\left(x_{0}, y\right) \varphi_{x_{0}}^{\Delta}(y) d A_{y} \\
& =\sum_{e \in I_{x_{0}}^{*}} \int_{\Gamma^{e}} H\left(x_{0}, y\right) \varphi_{x_{0}}^{\Delta}(y) d A_{y}-\int_{\Gamma_{x_{0}}^{*}} u_{x_{0}}^{\mathrm{polar}}(0, \widehat{\xi}(y)) D \varphi\left(x_{0}\right) T_{x_{0}}(y) d A_{y}, \\
& =\sum_{e \in I_{x_{0}}^{*}} \int_{\Gamma^{e}} U_{x_{0}, \varphi}(y) d A_{y}-\int_{\Gamma_{x_{0}}^{*, \Delta}} u_{x_{0}}^{\mathrm{polar}}(0, \widehat{\xi}(y)) D \varphi\left(x_{0}\right) T_{x_{0}}(y) d A_{y} .
\end{aligned}
$$

Substituting (164) and (163) into (161), and using the fact that $U_{x_{0}, \varphi}\left(x_{q}^{e}\right)=0$ if $x_{q}^{e}=x_{0}$ by Lemma 4.6(i), we get the decomposition

$$
\left(\mathcal{H}_{n} \varphi-\mathcal{H} \varphi\right)\left(x_{0}\right)=\mathcal{N}_{1}\left(x_{0}\right)+\mathcal{N}_{2}\left(x_{0}\right)-\mathcal{N}_{3}\left(x_{0}\right)+\mathcal{N}_{4}\left(x_{0}\right)+\mathcal{N}_{5}\left(x_{0}\right),
$$

where

$$
\begin{gathered}
\mathcal{N}_{1}\left(x_{0}\right)=\sum_{e \in I_{x_{0}}^{*}}\left[\sum_{q=1}^{Q} U_{x_{0}, \varphi}\left(x_{q}^{e}\right) W_{q}^{e}-\int_{\Gamma^{e}} U_{x_{0}, \varphi}(y) d A_{y}\right], \\
\mathcal{N}_{2}\left(x_{0}\right)=\sum_{e \in I_{x_{0}}^{*}} \sum_{1 \leq q \leq Q}^{\prime} u_{x_{0}}^{\text {polar }}\left(0, \widehat{\xi}\left(x_{q}^{e}\right)\right) D \varphi\left(x_{0}\right) T_{x_{0}}\left(x_{q}^{e}\right) W_{q}^{e} \\
+\int_{\Gamma_{x_{0}}^{*, \Delta}} u_{x_{0}}^{\text {polar }}(0, \widehat{\xi}(y)) D \varphi\left(x_{0}\right) T_{x_{0}}(y) d A_{y}, \\
\mathcal{N}_{3}\left(x_{0}\right)=\sum_{e \in I_{x_{0}}^{*}} \sum_{1 \leq q \leq Q}^{\prime} \widehat{\zeta}_{q}^{e}\left(x_{0}\right) H\left(x_{0}, x_{q}^{e}\right) \varphi_{x_{0}}^{\Delta}\left(x_{q}^{e}\right) W_{q}^{e},
\end{gathered}
$$


(169)

$$
\begin{gathered}
\mathcal{N}_{4}\left(x_{0}\right)=\sum_{e \notin I_{x_{0}}^{*}}\left[\sum_{q=1}^{Q} \zeta_{q}^{e}\left(x_{0}\right) H\left(x_{0}, x_{q}^{e}\right) \varphi_{x_{0}}^{\Delta}\left(x_{q}^{e}\right) W_{q}^{e}-\int_{\Gamma^{e}} H\left(x_{0}, y\right) \varphi_{x_{0}}^{\Delta}(y) d A_{y}\right], \\
\mathcal{N}_{5}\left(x_{0}\right)=\sum_{b=1}^{n} \widehat{\zeta}_{b}\left(x_{0}\right) R_{x_{0}}\left(x_{b}\right) \varphi_{x_{0}}^{\Delta}\left(x_{b}\right) .
\end{gathered}
$$

To establish the result, we proceed to bound each of $\mathcal{N}_{1}, \ldots, \mathcal{N}_{5}$ uniformly in $x_{0}$.

For the term $\mathcal{N}_{1}$, we note that $\Gamma^{e} \subset \Gamma_{x_{0}}^{*} \subset \Gamma_{x_{0}, d}$ for all $e \in I_{x_{0}}^{*}$ by definition, and $U_{x_{0}, \varphi} \in C^{0,1}\left(\Gamma_{x_{0}, d}, \mathbb{R}^{k}\right)$ with a Lipschitz constant that is uniform in $x_{0}$ by Lemma 4.6(i). In view of the quadrature error bound in (A6) for a rule of order $\ell$, we get

$$
\begin{aligned}
\left|\mathcal{N}_{1}\left(x_{0}\right)\right| & \leq \sum_{e \in I_{x_{0}}^{*}}\left|\sum_{q=1}^{Q} U_{x_{0}, \varphi}\left(x_{q}^{e}\right) W_{q}^{e}-\int_{\Gamma^{e}} U_{x_{0}, \varphi}(y) d A_{y}\right| \\
& \leq C_{\varphi} h^{\min (\ell, 1)}=C_{\varphi} h, \quad \forall n \geq N, \quad x_{0} \in \Gamma .
\end{aligned}
$$

For the term $\mathcal{N}_{2}$, we use Lemma 4.6(ii)(c) and (137) to conclude directly that

$$
\left|\mathcal{N}_{2}\left(x_{0}\right)\right| \leq C_{\varphi} h, \quad \forall n \geq N, \quad x_{0} \in \Gamma .
$$

For the term $\mathcal{N}_{3}$, we use the same arguments that led to (158) to get

$$
\left|\mathcal{N}_{3}\left(x_{0}\right)\right| \leq C_{\varphi} h^{\min (p+2, m+2)}=C_{\varphi} h^{2}, \quad \forall n \geq N, \quad x_{0} \in \Gamma .
$$

For the term $\mathcal{N}_{5}$, we use the same arguments that led to (156) to get

$$
\left|\mathcal{N}_{5}\left(x_{0}\right)\right| \leq C_{\varphi} h^{\min (p+1, m+1)}=C_{\varphi} h, \quad \forall n \geq N, \quad x_{0} \in \Gamma .
$$

In the above, the constant $C_{\varphi}$ depends on $\varphi$, but is independent of $x_{0}$. Thus uniform bounds for $\mathcal{N}_{1}, \mathcal{N}_{2}, \mathcal{N}_{3}$ and $\mathcal{N}_{5}$ are established.

It remains to bound the term $\mathcal{N}_{4}$. For every $x_{0} \in \Gamma$, we note from Lemma 4.6(ii)(a) that the set $\Gamma_{x_{0}}^{*}$ contains the patch $\Gamma_{x_{0}, d / C}$. Since $\operatorname{diam}\left(\Gamma^{e}\right) \leq h$ and $\operatorname{diam}\left(\operatorname{supp}\left(\widehat{\zeta}_{q}^{e}\right)\right) \leq C h$, we find, after increasing the size of $N$ if necessary, that $\frac{d}{2 C} \leq \frac{d}{C}-h \leq\left|y-x_{0}\right|, \widehat{\zeta}_{q}^{e}\left(x_{0}\right)=0$ and $\zeta_{q}^{e}\left(x_{0}\right)=1$ for all $y \in \Gamma^{e}, e \notin I_{x_{0}}^{*}$, $q=1, \ldots, Q, n \geq N$ and $x_{0} \in \Gamma$. Using these observations, and the fact that $H\left(x_{0}, y\right)$ is class $C^{m, 1}$ on the set $\left|y-x_{0}\right| \geq d /(2 C)$ by (A2), and $\varphi_{x_{0}}^{\Delta}$ is class $C^{m, 1}$ by (145), both with Lipschitz constants for each derivative uniform in $x_{0}$, together with the quadrature error bound in (A6) for a rule of order $\ell$, we get

$$
\begin{aligned}
\left|\mathcal{N}_{4}\left(x_{0}\right)\right| & \leq \sum_{e \notin I_{x_{0}}^{*}}\left|\sum_{q=1}^{Q}\left(H_{x_{0}} \varphi_{x_{0}}^{\Delta}\right)\left(x_{q}^{e}\right) W_{q}^{e}-\int_{\Gamma^{e}}\left(H_{x_{0}} \varphi_{x_{0}}^{\Delta}\right)(y) d A_{y}\right| \\
& \leq C_{\varphi} h^{\min (\ell, m+1)}, \quad \forall n \geq N, \quad x_{0} \in \Gamma .
\end{aligned}
$$

As before, the constant $C_{\varphi}$ depends on $\varphi$, but is independent of $x_{0}$. Thus a uniform bound for $\mathcal{N}_{4}$ is established. Combining (175), (174), (173), (172), (171) and (165), we obtain the bound

$$
\left\|\mathcal{H}_{n} \varphi-\mathcal{H} \varphi\right\| \leq C_{\varphi} h, \quad \forall n \geq N
$$

Combining (176), (142), (140) and (138), we get

$$
\left\|\varphi_{n}-\varphi\right\| \leq C_{\varphi} h \quad \text { as } \quad n \rightarrow \infty, \quad \forall \ell \geq 1, p=0, m \geq 1 .
$$

Thus the result stated in part (ii) of the main theorem is established. 


\section{REFERENCES}

[1] S.A. Allison and S. Mazur, Modeling the free solution electrophoretic mobility of short DNA Fragments, Biopolymers 46 (1998), 359-373.

[2] B. Alpert, G. Beylkin, R. Coifman, and V. Rokhlin, Wavelet-like bases for the fast solution of second-kind integral equations, SIAM J. Sci. Comput. 14 (1993), no. 1, 159-184, DOI 10.1137/0914010. MR1201316 (93i:65126)

[3] Philip M. Anselone, Collectively Compact Operator Approximation Theory and Applications to Integral Equations, Prentice-Hall Inc., Englewood Cliffs, N. J., 1971. With an appendix by Joel Davis; Prentice-Hall Series in Automatic Computation. MR0443383 (56 \#1753)

[4] P. M. Anselone, Singularity subtraction in the numerical solution of integral equations, J. Austral. Math. Soc. Ser. B 22 (1980/81), no. 4, 408-418, DOI 10.1017/S0334270000002757. MR626932(82h:65095)

[5] S. Aragon and D.K. Hahn, Precise boundary element computation of protein transport properties: diffusion tensors, specific volume and hydration, Biophys. J. 91 (2006), 1591-1603.

[6] K. E. Atkinson, The numerical solution of Laplace's equation in three dimensions, SIAM J. Numer. Anal. 19 (1982), no. 2, 263-274, DOI 10.1137/0719017. MR650051 (83d:65276)

[7] Kendall E. Atkinson, The Numerical Solution of Integral Equations of the Second Kind, Cambridge Monographs on Applied and Computational Mathematics, vol. 4, Cambridge University Press, Cambridge, 1997. MR 1464941 (99d:65364)

[8] J.P. Bardhan, Numerical solution of boundary-integral equations for molecular electrostatics, J. Chem. Phys. 130 (2009), 094102.

[9] C.A. Brebbia, J.C.F. Telles, and L.C. Wrobel, Boundary Element Techniques: Theory and Applications in Engineering, Springer-Verlag, Berlin, 1984.

[10] D. Brune and S. Kim, Predicting protein diffusion coefficients, Proc. Natl. Acad. Sci. USA 90 (1993), 3835-3839.

[11] O.P. Bruno, V. Dominguez, and F.-J. Sayas, Convergence analysis of a high-order Nyström integral-equation method for surface scattering problems, submitted, arXiv:1109.6352.

[12] Oscar P. Bruno and Leonid A. Kunyansky, A fast, high-order algorithm for the solution of surface scattering problems: basic implementation, tests, and applications, J. Comput. Phys. 169 (2001), no. 1, 80-110, DOI 10.1006/jcph.2001.6714. MR.1829284(2002b:65188)

[13] Lawrence F. Canino, John J. Ottusch, Mark A. Stalzer, John L. Visher, and Stephen M. Wandzura, Numerical solution of the Helmholtz equation in $2 D$ and $3 D$ using a high-order Nyström discretization, J. Comput. Phys. 146 (1998), no. 2, 627-663, DOI 10.1006/jcph.1998.6077. MR.1654932

[14] Goong Chen and Jianxin Zhou, Boundary Element Methods, Computational Mathematics and Applications, Academic Press Ltd., London, 1992. MR.1170348 (93e:65143)

[15] David Colton and Rainer Kress, Inverse Acoustic and Electromagnetic Scattering Theory, 2nd ed., Applied Mathematical Sciences, vol. 93, Springer-Verlag, Berlin, 1998. MR1635980 (99c:35181)

[16] Michael G. Duffy, Quadrature over a pyramid or cube of integrands with a singularity at a vertex, SIAM J. Numer. Anal. 19 (1982), no. 6, 1260-1262, DOI 10.1137/0719090. MR679664 (83k:65020)

[17] M. Ganesh and I. G. Graham, A high-order algorithm for obstacle scattering in three dimensions, J. Comput. Phys. 198 (2004), no. 1, 211-242, DOI 10.1016/j.jcp.2004.01.007. MR2071394(2005d:65217)

[18] M. Ganesh, I. G. Graham, and J. Sivaloganathan, A new spectral boundary integral collocation method for three-dimensional potential problems, SIAM J. Numer. Anal. 35 (1998), no. 2, 778-805, DOI 10.1137/S0036142996301238. MR1618899(99b:65173)

[19] M. A. Golberg and C. S. Chen, Discrete Projection Methods for Integral Equations, Computational Mechanics Publications, Southampton, 1997. MR1445293 (98c:45002)

[20] O. Gonzalez, On stable, complete, and singularity-free boundary integral formulations of exterior Stokes flow, SIAM J. Appl. Math. 69 (2009), no. 4, 933-958, DOI 10.1137/070698154. MR2476585(2010b:35364)

[21] O. Gonzalez and J. Li, Modeling the sequence-dependent diffusion coefficients of short DNA sequences, J. Chem. Phys. 129 (2008), 165105. 
[22] I. G. Graham and I. H. Sloan, Fully discrete spectral boundary integral methods for Helmholtz problems on smooth closed surfaces in $\mathbb{R}^{3}$, Numer. Math. 92 (2002), no. 2, 289-323, DOI 10.1007/s002110100343. MR1922922 (2003h:65179)

[23] N. M. Günter, Potential Theory and Its Applications to Basic Problems of Mathematical Physics, Translated from the Russian by John R. Schulenberger, Frederick Ungar Publishing Co., New York, 1967. MR0222316 (36 \#5368)

[24] Wolfgang Hackbusch, Integral Equations: Theory and Numerical Treatment, International Series of Numerical Mathematics, vol. 120, Birkhäuser Verlag, Basel, 1995. Translated and revised by the author from the 1989 German original. MR 1350296 (96h:45001)

[25] F.-K. Hebeker, A boundary element method for Stokes equations in 3-D exterior domains, The mathematics of finite elements and applications, V (Uxbridge, 1984), Academic Press, London, 1985, pp. 257-263. MR811037 (86m:76034)

[26] George C. Hsiao and Wolfgang L. Wendland, Boundary Integral Equations, Applied Mathematical Sciences, vol. 164, Springer-Verlag, Berlin, 2008. MR2441884 (2009i:45001)

[27] M. A. Jaswon and G. T. Symm, Integral Equation Methods in Potential Theory and Elastostatics, Academic Press [Harcourt Brace Jovanovich Publishers], London, 1977. Computational Mathematics and Applications. MR0499236 (58 \#17147)

[28] L. V. Kantorovich and V. I. Krylov, Approximate Methods of Higher Analysis, Translated from the 3rd Russian edition by C. D. Benster, Interscience Publishers, Inc., New York, 1958. MR0106537 (21 \#5268)

[29] O.D. Kellogg, Foundations of Potential Theory, Frederick Ungar Publishing, New York, 1929.

[30] S. Kim and S.J. Karrila, Microhydrodynamics: Principles and Selected Applications, Butterworth Heinemann Publishing, Boston, 1991.

[31] Rainer Kress, Linear Integral Equations, 2nd ed., Applied Mathematical Sciences, vol. 82, Springer-Verlag, New York, 1999. MR1723850 (2000h:45001)

[32] Alexander Kurganov and Jeffrey Rauch, The Order of accuracy of quadrature formulae for periodic functions, Advances in phase space analysis of partial differential equations, Progr. Nonlinear Differential Equations Appl., vol. 78, Birkhäuser Boston Inc., Boston, MA, 2009, pp. 155-159, DOI 10.1007/978-0-8176-4861-9_9. MR2664609 (2011g:65028)

[33] O. A. Ladyzhenskaya, The Mathematical Theory of Viscous Incompressible Flow, Revised English edition. Translated from the Russian by Richard A. Silverman, Gordon and Breach Science Publishers, New York, 1963. MR0155093 (27 \#5034b)

[34] Christian Lage and Christoph Schwab, Wavelet Galerkin algorithms for boundary integral equations, SIAM J. Sci. Comput. 20 (1999), no. 6, 2195-2222 (electronic), DOI 10.1137/S1064827597329989. MR1703278(2000e:65113)

[35] J. Li, A computational model for the diffusion coefficients of DNA with applications, The University of Texas, Austin, 2010, Ph.D. dissertation.

[36] J. Li and O. Gonzalez, Convergence and conditioning of a Nyström method for Stokes flow in exterior three-dimensional domains, Adv. Comput. Math. 39 (2013), no. 1, 143-174, DOI 10.1007/s10444-012-9272-1. MR3068598

[37] A.M. Linkov, Boundary Integral Equations in Elasticity Theory, Solid Mechanics and Its Applications, Volume 99, Kluwer Academic Publishers, Boston, 2002.

[38] S. G. Mihlin, Linear Integral Equations, Russian Monographs and Texts on Advanced Mathematics and Physics, Vol. I, Gordon and Breach Publishers, Inc., New York, 1960. MR0133657 (24 \#A3483)

[39] Jean-Claude Nédélec, Acoustic and Electromagnetic Equations: Integral Representations for Harmonic Problems, Applied Mathematical Sciences, vol. 144, Springer-Verlag, New York, 2001. MR.1822275 (2002c:35003)

[40] F. K. G. Odqvist, Uber die Randwertaufgaben der Hydrodynamik zäher Flüssigkeiten (German), Math. Z. 32 (1930), no. 1, 329-375, DOI 10.1007/BF01194638. MR.1545170

[41] Henry Power and Guillermo Miranda, Second kind integral equation formulation of Stokes' flows past a particle of arbitrary shape, SIAM J. Appl. Math. 47 (1987), no. 4, 689-698, DOI 10.1137/0147047. MR898827(88h:76021)

[42] H. Power and L.C. Wrobel, Boundary Integral Methods in Fluid Mechanics, Computational Mechanics Publications, Southampton, 1995.

[43] C. Pozrikidis, Boundary Integral and Singularity Methods for Linearized Viscous Flow, Cambridge Texts in Applied Mathematics, Cambridge University Press, Cambridge, 1992. MR.1156495(93a:76027) 
[44] A. Rathsfeld, Quadrature methods for 2D and 3D problems: Numerical analysis 2000, Vol. VI, Ordinary differential equations and integral equations, J. Comput. Appl. Math. 125 (2000), no. 1-2, 439-460, DOI 10.1016/S0377-0427(00)00484-2. MR1803207 (2002h:65030)

[45] Sergej Rjasanow and Olaf Steinbach, The Fast Solution of Boundary Integral Equations, Mathematical and Analytical Techniques with Applications to Engineering, Springer, New York, 2007. MR2310663 (2008g:65179)

[46] A. H. Stroud, Approximate Calculation of Multiple Integrals, Prentice-Hall Inc., Englewood Cliffs, N.J., 1971. Prentice-Hall Series in Automatic Computation. MR0327006 (48 \#5348)

[47] Francesco Tricomi, Equazioni integrali contenenti il valor principale di un integrale doppio (Italian), Math. Z. 27 (1928), no. 1, 87-133, DOI 10.1007/BF01171089. MR.1544900

[48] Lexing Ying, George Biros, and Denis Zorin, A high-order 3D boundary integral equation solver for elliptic PDEs in smooth domains, J. Comput. Phys. 219 (2006), no. 1, 247-275, DOI 10.1016/j.jcp.2006.03.021. MR.2273377(2007k:65205)

Department of Mathematics, The University of Texas at Austin, Austin, Texas 78712

E-mail address: og@math.utexas.edu

Graduate Program in Computational and Applied Mathematics, The University of Texas at Austin, Austin, Texas 78712

Current address: Schlumberger Corporation, Houston, Texas

E-mail address: JLi49@slb.com 